\title{
Myelin lipids are energy reserves in the nervous system
}

\author{
Dissertation \\ for the award of the degree \\ "Doctor of Philosophy" \\ Division of Mathematics and Natural Sciences \\ of the Georg-August-Universität Göttingen \\ within the doctoral program Biology \\ of the Georg-August University School of Science (GAUSS) \\ submitted by \\ Ebrahim Asadollahi \\ Born in Kashmar, Iran
}

Göttingen, March 2020 


\section{Thesis Committee}

Prof. Dr. Klaus-Armin Nave

Department of Neurogenetics

Max-Planck-Institute of Experimental Medicine Göttingen

Prof. Dr. Peter Schu

Department of Cellular Biochemistry

University Medical Center Göttingen

Dr. Nuno Raimundo

Department of Cellular Biochemistry

University Medical Center Göttingen

Prof. Dr. Martin Göpfert

Department of Cellular Neurobiology

Schwann-Schleiden Research Centre - University of Göttingen

\section{Members of the Examination Board}

1. Prof. Dr. Dr. Hannelore Ehrenreich

Department of Clinical Neuroscience

Max-Planck-Institute of Experimental Medicine Göttingen

2. Prof. Dr. Ralf Heinrich

Department of Cellular Neurobiology

Schwann-Schleiden Research Centre - University of Göttingen

Date of oral examination: 17.04.2020 
declaration

I hereby declare that the $\mathrm{PhD}$ thesis entitled "Myelin lipids are energy reserves in the nervous system", was written independently and with no other sources and aids than quoted.

Göttingen, 06.03.2020

Ebrahim Asadollahi 


\section{Acknowledgments}

I would like to express my deepest gratitude to Prof. Klaus Nave for his full support through my Ph.D and providing me with the opportunity to benefit from his amazing knowledge in neuroscience and stimulating discussions that kept me motivated to seek for excellence.

I would like to express my appreciation to Dr. Celia Kassmann for guiding me at the beginning of my PhD and for all the discussions and providing me with the animals that helped me to start working on the project.

I am extremely grateful to Dr. Andrea Trevisiol for teaching me fancy technique for studying nerve function (Electrophysiology), Dr. Aiman Saab for teaching me the optic nerve incubation and his student (Zoe Looser) for providing me with samples.

I would like to express my appreciation to Dr. Payam Dibaj for his collaboration and his advices, Prof. E. Dale Abel, Prof. Andrea Ballabio, Prof. Johannes Hirrlinger and Dr. Gesine Saher for providing the mice, the proteomics lab (Dr. Olaf Jahn and Doerte Hesse) for performing proteomics, electron microscopy facility (Dr. Wiebke Möbius, Torben Ruhwedel and Boguslawa Sadowski for doing electron microscopy of the samples) and Kathrin Kusch for doing western blotting.

I would like to thank my annual thesis committee meeting members for their scientific input and their full support including Dr. Nuno Raimundo and Prof. Peter Schu that were present since the beginning of my studies and Prof. Martin Göpfert who kindly accepted to joined us later. I further would like to thank the other members of the examination board, Prof. Hannelore Ehrenreich and Prof. Ralf Heinrich for taking the time to evaluate my thesis. I like to thank the GAUSS program office for their support.

I am very grateful to all the people in MPI-EM facilitated doing my project during my PhD including: Sarah S., Ursula F., and Anke S (at the animal facility); Hajo H., Rolf M., (IT team); Harry S., (electronics support); Miso M. (at the LMF); Markus Krohn and his team (at the finemechanik department); Anette, Gudrun, Jenni, Ramona and Ulli (neurogenetics department). 
I would like to thank the people at the front desk for their help and also the game that we were usually playing with switching on and off the lights at midnight and also many thanks to Birgit, Anette and their coworkers who start their work very early in the morning to facilitate working in the lab.

Thanks to all the present and former members of Neurogenetics department: Alexandra, Chi, Clara, Constanze, David, Doris, Eric, Georg, Hugo, Iva, Jan, Katja, Lisa, Lena, Maria, Martin, Michelle, Sandra, Sarah, Sharlen, Sina, Sofia, Stefan, Swatti, Tamer, Theresa, Tilman, Tim, Tobias, Ulrike and all the group leaders in the lab for their effort in creating a vibrant atmosphere and nice working place.

I am very thankful to Gabriele and Michaela for their best effort in making my working in the lab as efficient as possible and for making me laugh with the daily jokes.

Special thanks to Alejandro, Andrea, Carmela and Ting for their comments on writing my dissertation and proofreading this thesis.

I am very grateful to DAAD foundation for the positive reply to my application for scholarship and giving me the chance of enjoying German technology.

At the end I would like to express many thanks to all my family members specially my wonderful parents and my little sister living with them, Marziyeh, for keeping supporting me from abroad. 



\section{Contents}

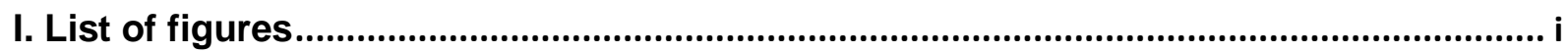

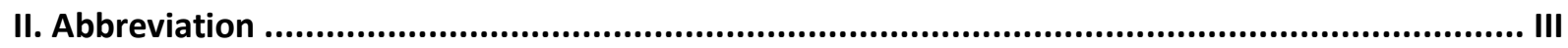

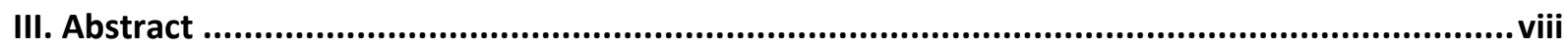

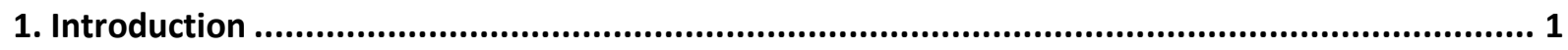

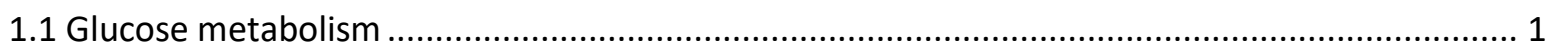

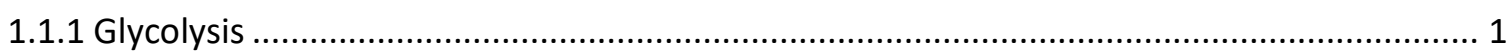

1.1.2 Tricarboxylic acid cycle and oxidative phosphorylation .................................................. 2

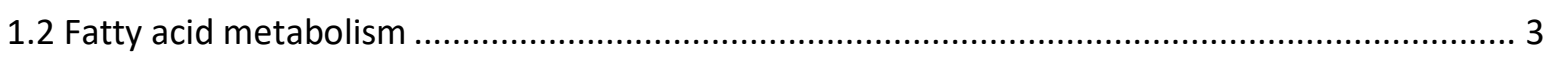

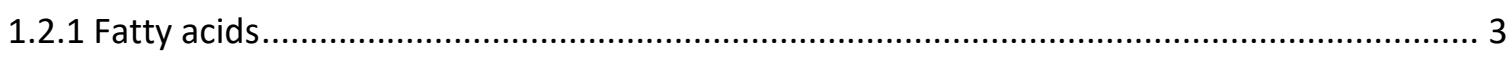

1.2.2 Extracellular and intracellular sources of fatty acids ....................................................... 3

1.2.3 $\beta$-Oxidation of fatty acids in mitochondria and peroxisomes ............................................ 5

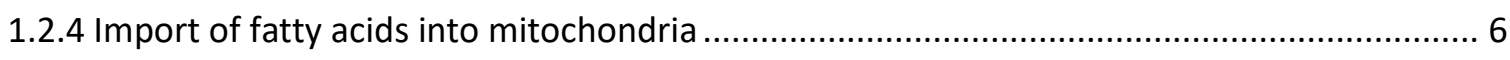

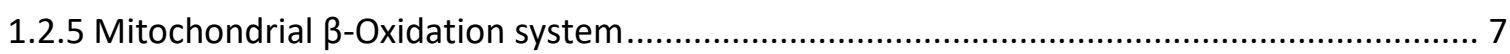

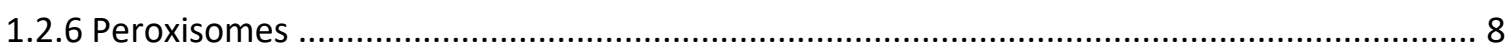

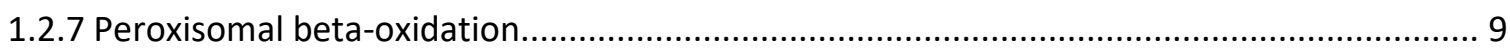

1.2.8 Interaction between peroxisomes and mitochondria .................................................... 10

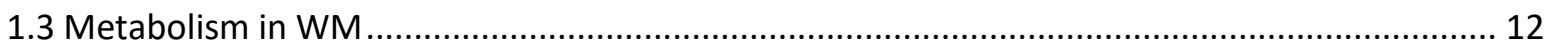

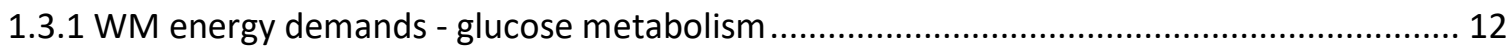

1.3.2 Effect of myelination on signal propagation and energy utilization in WM ........................ 14

1.3.3 WM energy demands- fatty acid metabolism ............................................................. 17

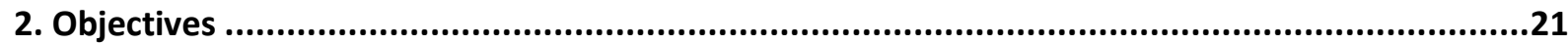

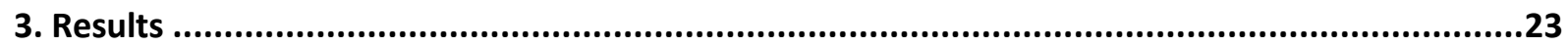

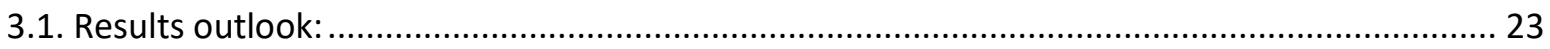

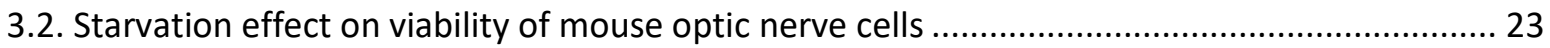

3.2.1. Evaluation of the mouse optic nerve as a model system ................................................. 23

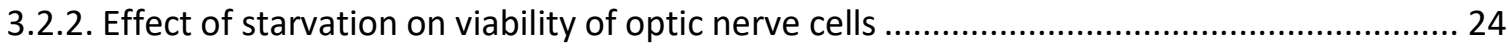

3.2.3. Effect of starvation on viability of different cell types in the optic nerve........................... 25

3.2.4. Why do astrocytes die in the absence of glucose? ............................................................. 27

3.3. Myelin-derived fatty acuds as a source of energy under starvation conditions ........................ 27

3.3.1. Effect of beta oxidation inhibitors on survival of optic nerve cells under starvation-

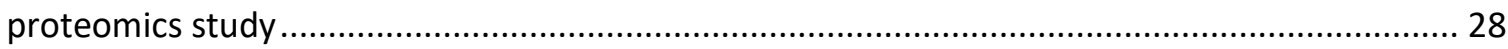

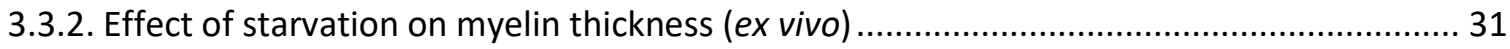

3.4. Role of fatty acid metabolism in supporting nerve function under starvation condition........... 33

3.4.1. Finding the threshold condition for optic nerve function ................................................ 34 
3.4.2. Beta-hydroxybutyrate supports nerve function under starvation condition.

3.4.3. Effect of blocking mitochondrial beta-oxidation on nerve function under starvation conditions

3.4.4. Effect of blocking peroxisomal beta-oxidation on nerve function under starvation condition (Thioridazine and Mfp2 KO mice)

3.5. Mechanism of myelin degradation (autophagy perturbation: Tfeb KO mice, Lys05) and transport of fatty acids (Octn3 KO mice).

3.6. Effect of glucose uptake perturbation (in vivo) in mature oligodendrocytes on myelin thickness

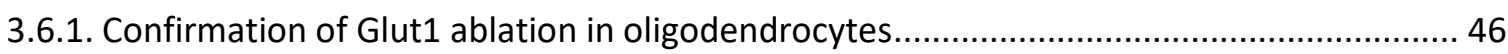

3.6.2. EM studies and g-ratio quantification in optic nerve of Glut1 ciKO mice .......................... 46

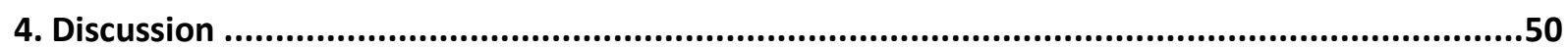

4.1. Susceptibility of different optic nerve cell types to starvation condition ................................. 50

4.2. Fatty acids are metabolized by optic nerve cells under starvation condition.............................. 51

4.3. Beta-oxidation in both mitochondria and peroxisomes is involved in supporting nerve function when glucose availability is restricted.

4.4. Beta-oxidation in myelin peroxisomes play an important role in supporting optic nerve function under starvation.

4.5. Investigation on mechanism of myelin degradation and export of peroxisomal beta-oxidation products

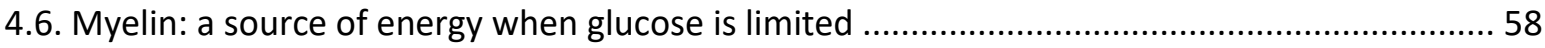

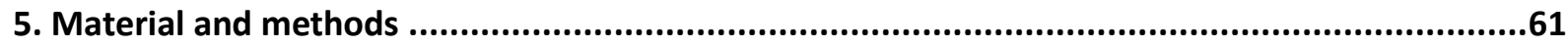

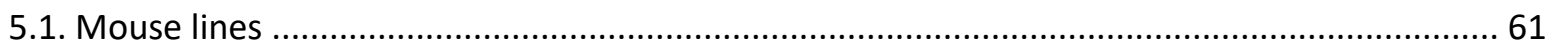

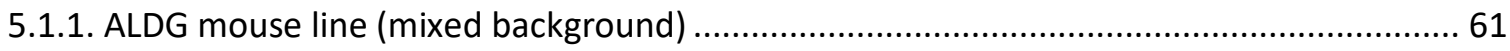

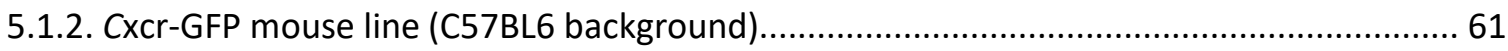

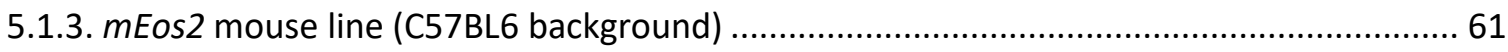

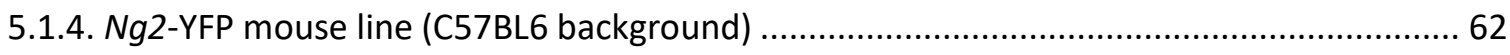

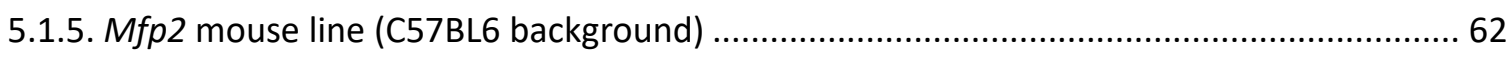

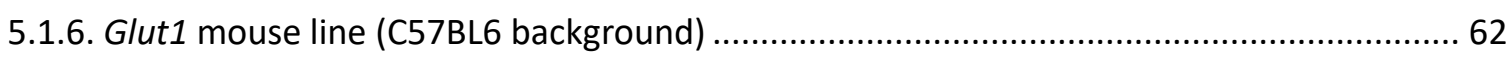

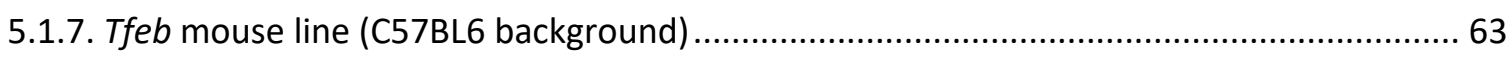

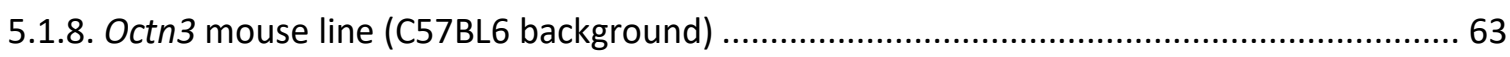

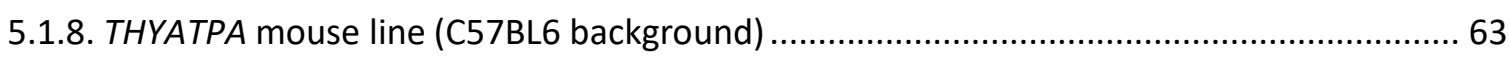

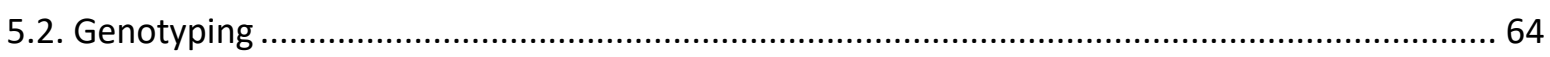

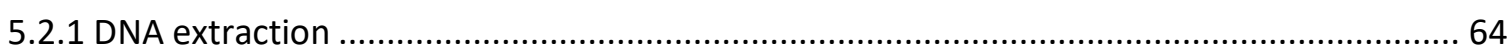

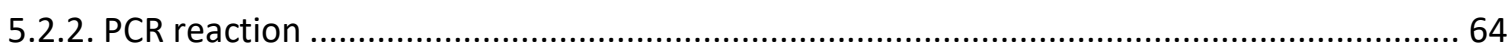

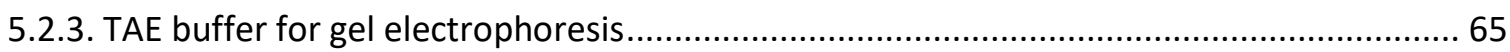

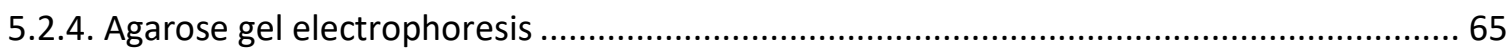

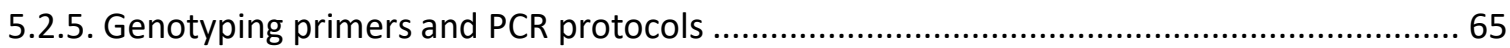




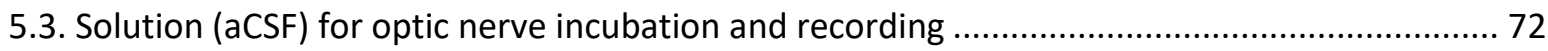

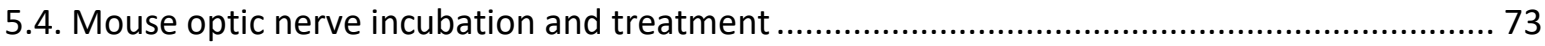

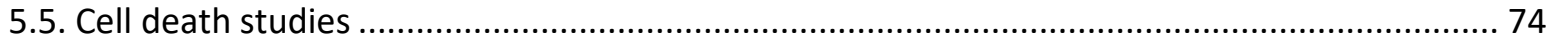

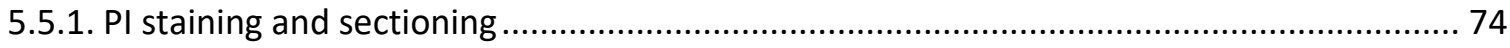

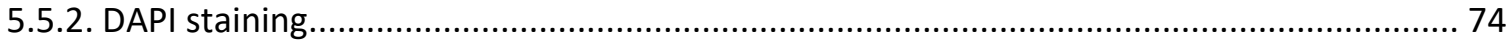

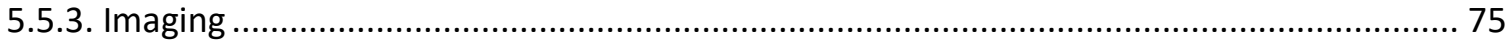

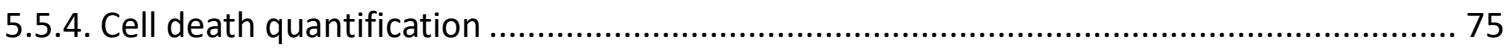

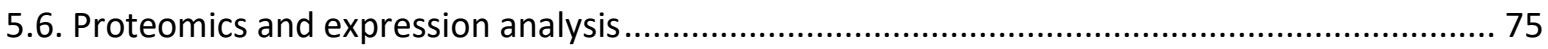

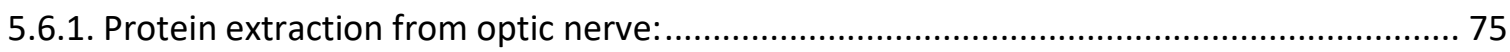

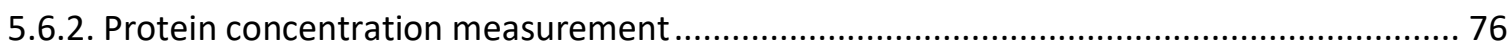

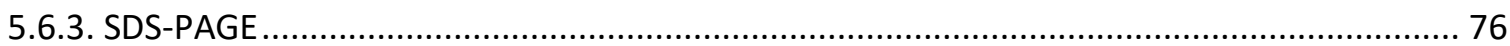

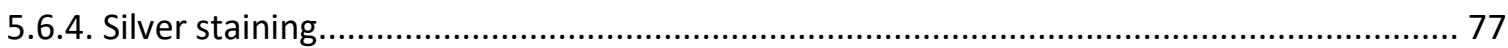

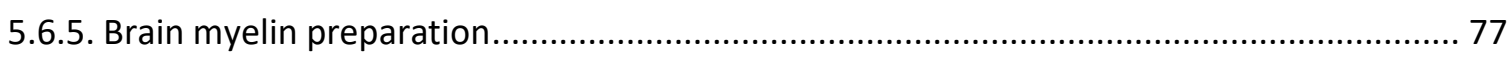

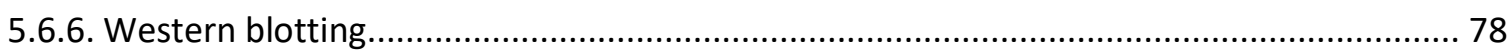

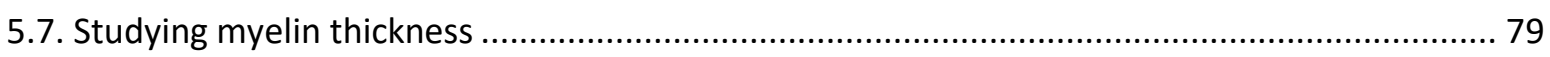

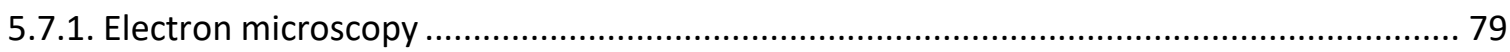

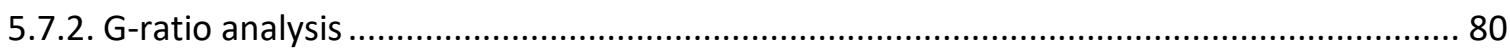

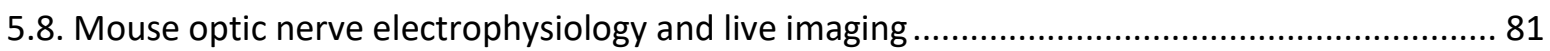

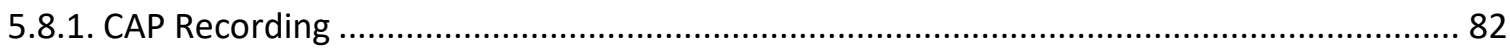

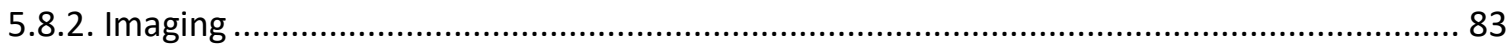

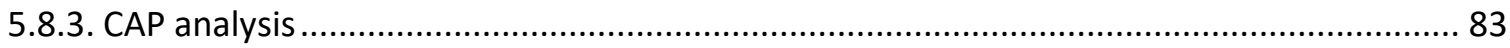

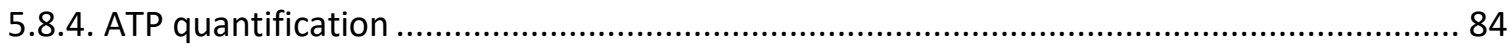

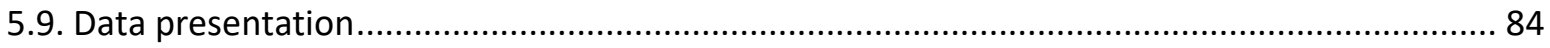

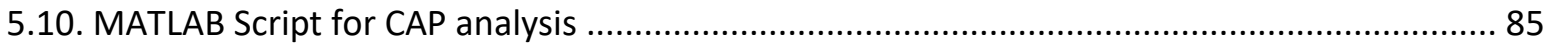

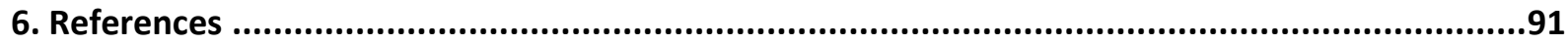

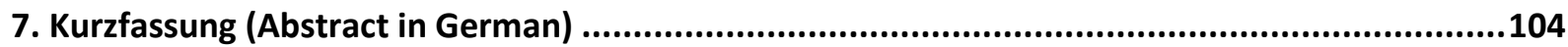




\section{List of figures}

Figure 1. Evaluation of the optic nerve preparation for incubation purpose.

Figure 2. Effects of 16 and $24 \mathrm{~h}$ starvation on viability of optic nerve cells

Figure 3. Effects of 24h starvation on frequency and survival of different cell types in mouse optic nerve.

Figure 4. Presence of $1 \mathrm{mM}$ glucose or $1.5 \mathrm{mM}$ beta-hydroxybutyrate is enough for supporting the survival of optic nerve cells.

Figure 5. Hypoxia effect on survival of optic nerve cells incubated in aCSF $+10 \mathrm{mM}$ glucose (Glc) / or $10 \mathrm{mM}$ sucrose (Stv) for $16 \mathrm{~h}$

Figure 6. Effect of mitochondrial beta-oxidation inhibition on survival of optic nerve cells incubated in aCSF+ $10 \mathrm{mM}$ glucose (Glc) / or $10 \mathrm{mM}$ sucrose (Stv) for $16 \mathrm{~h}$.

Figure 7. Effect of peroxisomal beta-oxidation inhibition on survival of optic nerve cells incubated in aCSF $+10 \mathrm{mM}$ glucose (Glc)/ or $10 \mathrm{mM}$ sucrose (Stv) for $16 \mathrm{~h}$.

Figure 8. Effect of $16 \mathrm{~h}$ starvation on protein expression profile of optic nerve cells (incubated in aCSF+ $10 \mathrm{mM}$ glucose (Glc)/ or $1 \mathrm{mM}$ glucose (Stv)).

Figure 9. Effect of $16 \mathrm{~h}$ starvation (aCSF $+10 \mathrm{mM}$ sucrose (Stv)) on thickness of myelin in acutely isolated optic nerve.

Figure 10. Optic nerve function at different concentrations of glucose obtained by ex vivo electrophysiology using suction electrodes to find the lowest glucose concentration needed nerve function. 35

Figure 11. Optic nerve function at different frequencies $(\mathrm{Hz})$ obtained by ex vivo electrophysiology using suction electrodes.

Figure 12. Support of optic nerve function by beta-hydroxybutyrate (HB) alone and in combination with glucose

Figure 13. Effect of mitochondrial beta-oxidation inhibitor $(25 \mu \mathrm{M} 4-\mathrm{Br})$ on optic nerve function under starvation obtained by ex vivo electrophysiology using suction electrodes. 38 
Figure 14. Effect of long chain FA beta-oxidation inhibition (5 $\mu \mathrm{M}$ Etomoxir) on optic nerve function under starvation obtained by ex vivo electrophysiology using suction electrodes 40

Figure 15. Effect of peroxisomal beta-oxidation inhibition using ( $5 \mu \mathrm{M}$ Thioridazine) on optic nerve function under starvation obtained by ex vivo electrophysiology using suction electrodes.

Figure 16. Effect of perturbing peroxisomal beta-oxidation in oligodendrocytes ( $\left.\mathrm{Mfp} 2^{\mathrm{fl} / f l} * \mathrm{Cnp}-\mathrm{Cr} \mathrm{e}^{+/}\right)$ on optic nerve function under starvation obtained by ex vivo electrophysiology using suction electrodes.

Figure 17. Effect of perturbing autophagy (( $\left.\mathrm{Tfeb}^{\mathrm{fl} / f / *} \mathrm{Cnp}-\mathrm{Cr} \mathrm{e}^{+-}\right)$and pharmacologically (Lys05)) and carnitine transporter in the membrane of oligodendrocyte peroxisomes on optic nerve function under starvation

Figure 18. Immunoblotting results for myelin fraction obtained from G/ut1 ciKO mice brain verifying ablation of GLUT1 transporter in oligodendrocytes.

Figure 19. G-ratio quantifications for optic nerves of G/ut1 ciKO mice. .47

Figure 20. Interaction between myelin peroxisomes and mitochondria in degradation of myelin FA under starvation.

Figure 21. Prepared mouse optic nerve attached to the eyeball for incubation experiments. showing an example of acutely isolated mouse optic nerve (wild-type) for incubation experiments

Figure 22. Showing a scheme of combined electrophysiology and confocal imaging for monitoring neuronal function and ATP level in optic nerve from mouse line expressing ATP sensor in their axons. 


\section{Abbreviation}

\begin{tabular}{|c|c|}
\hline$A B C D$ & ATP-binding cassette transporter (subfamily D) \\
\hline ACaa1a & 3-ketoacyl-CoA thiolase A, peroxisomal \\
\hline ACaa1b & 3-ketoacyl-CoA thiolase $B$, peroxisomal \\
\hline ACAD & Acyl-CoA dehydrogenase \\
\hline ACAT & Carnitine acyl carnitine translocase \\
\hline ACOX & Acyl-CoA oxidase \\
\hline aCSF & Artificial creberospinal fluid \\
\hline ALDH1L1 & Aldehyde dehydrogenase 1 family, member L1 \\
\hline ALS & Amiotrophic lateral sclerosis \\
\hline AP & Action potential \\
\hline ATG3 & Autophagy related protein 3 (Ubiquitin-Like-Conjugating Enzyme ATG3) \\
\hline ATP & Adenosin triphosphate \\
\hline bp & Base pair \\
\hline $1,3 \mathrm{BPG}$ & 1,3 Bisphosphoglycerate \\
\hline $4-\mathrm{Br}$ & 4-Bromocrotonic acid \\
\hline${ }^{\circ} \mathrm{C}$ & Degrees celsius \\
\hline CAP & Compound action potential \\
\hline CA2 & Carbonic anhydrase \\
\hline KO & Conditional knockout \\
\hline ciKO & Conditional inducible knockout \\
\hline CNP & 2',3'-Cyclic nucleotide 3'-phosphodiesterase \\
\hline CNS & Centeral nervous system \\
\hline $\mathrm{CPT}$ & Carnitine palmitoyl transferase \\
\hline CRAT & Carnitine acetyl transferase \\
\hline CROT & Carnitineoctanoyl transferase \\
\hline CTR & Control \\
\hline DAPI & 4',6-diamidino-2-phenylindole \\
\hline dNTP & Deoxynucleotide triphosphate \\
\hline$\varnothing$ & Diameter \\
\hline EM & Electron microscopy \\
\hline ETC & Electron transport chain \\
\hline ETOX & Etomoxir \\
\hline FA & Fatty acid \\
\hline
\end{tabular}




\begin{tabular}{|c|c|}
\hline FABP & Fatty acid binding protein \\
\hline FABPpm & Fatty acid binding protein (plasma memberane) \\
\hline $\mathrm{FAD}+, \mathrm{FADH} 2$ & Flavin adenine dinucleotide $(\mathrm{H}$, protonated) \\
\hline FAT & Fatty acid translocase \\
\hline FFA & Free fatty acid \\
\hline FRET & Fluorescence resonance energy transfer \\
\hline GA3P & Glyceraldehyde 3- Phosphate \\
\hline GFAP & Glial fibrillary acidic protein \\
\hline Glc & Glucose \\
\hline GM & Gray matter \\
\hline $\mathrm{h}$ & Hour \\
\hline $\mathrm{HB}$ & Beta- hydroxybutyrate \\
\hline $\mathrm{Hz}$ & Hertz \\
\hline LAMP1 & Lysosomal-associated membrane protein 1 \\
\hline LAMP2 & Lysosomal-associated membrane protein 2 \\
\hline LIMP2 & Lysosomal integral membrane protein 2 \\
\hline LCAD & Long chain acyl-CoA dehydrogenase \\
\hline LCFA & Long chain fatty acid \\
\hline LCHAD & Long chain-hydroxyacyl-CoA dehydrogenase \\
\hline LCKAT & Long chain triketoacyl-CoA thiolase (part of MTP) \\
\hline LD & Lipid droplet \\
\hline LDH & Lactate dehydrogenase \\
\hline Lys05 & Autophagy inhibitor \\
\hline MCAD & Medium chain acyl-CoA dehydrogenase \\
\hline MCKAT & Medium chain triketoacyl-CoA thiolase \\
\hline MCFA & Medium chain fatty acid \\
\hline $\mathrm{MCT}$ & Monocarboxylate transporter \\
\hline MFP (1 and 2) & Peroxisomal multifunctional protein ( 1 and 2 ) \\
\hline $\mathrm{mg} / \mathrm{kg}$ & Miligram/kilogram \\
\hline $\mathrm{ml}$ & mililiter \\
\hline$\mu l$ & Microliter \\
\hline$\mu \mathrm{M}$ & Micromolar \\
\hline$\mu \mathrm{m}$ & Micrometer \\
\hline MON & Mouse optic nerve \\
\hline MTP & Mitochondrial trifunctional protein \\
\hline
\end{tabular}




\begin{tabular}{|c|c|}
\hline $\mathrm{mv}$ & Milivolt \\
\hline $\mathrm{NAD}+, \mathrm{NADH}$ & Nicotinamide adenine dinucleotide $(H$, protonated $)$ \\
\hline NMDA & N-methyl-D-aspartate \\
\hline GBRL2 & Gamma-aminobutyric acid receptor-associated protein-like 2 \\
\hline GFP & Green fluorescent protein \\
\hline GLUT1 & Facilitated glucose transporter, member1 (SLC2A1) \\
\hline $\mathrm{gr}$ & Gram \\
\hline OCTN2 & Organic cation transporter novel 2 \\
\hline OCTN3 & Organic cation transporter novel 3 \\
\hline Oligos & Oligodendrocytes \\
\hline OPCs & Oligodendrocyte precursor cells \\
\hline OX-PHOS & Oxidative phosphorylation \\
\hline PCR & Polymerase chain reaction \\
\hline PEP & phosphoenolpyruvate \\
\hline PFK1 & Phosphofructokinase 1 \\
\hline$P$ & P-value \\
\hline $\mathrm{PI}$ & Propidium iodide \\
\hline PTS1 & Peroxisomal targeting sequence 1 \\
\hline PVDF & Polyvinylidene fluoride \\
\hline RAB7a & Ras-related protein Rab-7a \\
\hline ROS & Reactive Oxygen species \\
\hline RT & Room temprature \\
\hline SCAD & Short chain acyl-CoA dehydrogenase \\
\hline SCFA & Short chain fatty acid \\
\hline SCHAD & Short chain-hydroxyacyl-CoA dehydrogenase \\
\hline SDS-PAGE & sodium dodecyl sulfate-polyacrylamide gel electrophoresis \\
\hline Sec & Second \\
\hline SEM & Standard error mean \\
\hline Stv & Starvation \\
\hline TAE & Tris-acetate-EDTA \\
\hline $\mathrm{TAG}(\mathrm{s})$ & Triacylglycerol(s) \\
\hline TCA & Tricarboxcylic acid \\
\hline TFEB & Transcription factor EB \\
\hline Thio & Thioridazine \\
\hline TUB & Alpha-tubulin \\
\hline
\end{tabular}




$\begin{array}{ll}\text { UV } & \text { Ultraviolet } \\ \text { v/v } & \text { Volume/volume } \\ \text { VLCFA } & \text { Very long chain fatty acid } \\ \text { WM } & \text { White matter } \\ \text { w/v } & \text { Weight/volume } \\ \text { YFP } & \text { Yellow fluorescent protein }\end{array}$

International System of Units (SI) was used throughout this thesis. 


\section{Abstract}

Neuronal functions critically depend on a continuous supply of glucose as prolonged hypoglycemia can lead to severe brain injury. It is surprising that except for astroglial glycogen the brain has no visible energy reserves. Myelin is a lipid-rich membrane that enwraps axons for fast impulse conduction. Myelinating oligodendrocytes also provide metabolic support to axons. We thus hypothesized that under starvation conditions myelin itself might support the white matter energy balance. Using the mouse optic nerve as a model, we found that oligodendrocytes but not astrocytes survive glucose deprivation ex vivo for up to 24 hours. In addition, myelin thickness was observed to decrease under starvation conditions and betaoxidation inhibition was shown to induce massive cell death in the optic nerve. These observations suggest fatty acids (FAs) released from myelin are involved in supporting cell survival under starvation Importantly, using electrophysiology combined with live imaging we showed that FA metabolism supports axonal ATP production and nerve conduction by perturbing beta-oxidation. This axonal support depends on FA beta-oxidation involving peroxisomes in the myelin compartment. To study reduced glucose availability in live mice, we deleted GLUT1 (SIc2a1) from mature oligodendrocytes, which led to slowly progressive loss of myelin. We therefore suggest a revised model for the dual function of oligodendrocytes, with myelin constituting an energy buffer that can prevent axonal degeneration when glucose availability is perturbed. These findings may be relevant for myelin abnormalities found in a range of neurodegenerative disease. 


\section{Introduction}

\subsection{Glucose metabolism}

\subsubsection{Glycolysis}

Glycolysis produces ATP, the energy currency of the cells, by breaking down glucose into two molecules of pyruvate. Glycolysis, an oxygen-independent process, takes place in the cytosol of the cell and it is known to be the only way a cell produces ATP without the need for mitochondrial oxidation. Glucose, the main source of energy for most of the cells, is taken up and transported in their cytosol via glucose transporters (GLUT1-5). As soon as glucose enters the cell, one molecule of ATP is readily used by the enzyme hexokinase to phosphorylate glucose on its $\mathrm{C} 6$ and glucose 6-phosphate is produced. When phosphorylated, glucose cannot leave the cell because of the newly acquired negative charge and, because of its different molecular composition, it maintains glucose gradient across the cell. Glucose 6-phosphate can 1) be stored into glycogen 2) go through glycolysis for ATP production or 3) enter the pentose phosphate pathway. Following the glycolytic path, glucose 6-phosphate is subsequently converted to fructose 6-phosphate that is in turn phosphorylated by phosphofructokinase (PFK1, a key enzyme that regulates glycolysis), by consumption of another molecule of ATP: fructose 1,6-bisphosphate is hence formed. Then, enolase converts fructose 1,6-bisphosphate into 2 molecules of glyceraldehyde 3-phosphate. In the following step, glyceraldehyde 3phosphate dehydrogenase produces 1,3-bisphosphoglycerate (1,3-BPG) from glyceraldehyde 3-phosphate. During this conversion, two molecules of $\mathrm{NADH}$ are produced by the coreduction of $\mathrm{NAD}^{+}$. The conversion of 1,3-BPG to 3-phosphoglycerate goes along with the production of two molecules of ATP. Acting phosphoglycerate mutase and enolase result in the formation of phosphoenolpyruvate from 3-phosphoglycerate and, in the last step of glycolysis, two more ATP molecules are produced, upon conversion of phosphoenolpyruvate to pyruvate. In glycolysis, per each molecule of glucose, two ATP molecules are consumed and four molecules of ATP, two molecules of NADH and two molecules of pyruvate are produced (Jeremy M Berg, John L Tymoczko, 2002) (Fig. I). 


\subsubsection{Tricarboxylic acid cycle and oxidative phosphorylation}

In the absence of oxygen, the glycolytic product, pyruvate, can be converted into lactate or ethanol depending on the organism. However, under aerobic conditions, pyruvate, can be transported into mitochondria for further oxidation and ATP production: the process is known as oxidative phosphorylation (OX-PHOS). Pyruvate transport into mitochondria is operated by the pyruvate carrier, an antiporter that transfers pyruvate from the cytoplasm into the mitochondria matrix in exchange for $\mathrm{OH}^{-}$. In the matrix of mitochondria, the pyruvate dehydrogenase complex converts pyruvate into one molecule of $\mathrm{CO}_{2}$, one of acetyl-CoA and one molecule of $\mathrm{NADH}$ through reduction of $\mathrm{NAD}^{+}$. The produced acetyl-CoA, in turn, enters the tricarboxylic acid (TCA) cycle upon condensation with oxaloacetate and formation of citric acid, a reaction that is catalyzed by citrate synthase. The cycle continues by isomerization of citrate to isocitrate that, following two decarboxylation steps, is converted into alphaketoglutarate and succinyl-CoA respectively. In total, two molecules of $\mathrm{CO}_{2}$ and two molecules of $\mathrm{NADH}$ are generated. Then succinate is detached from -CoA, a reaction that produces one molecule of GTP (that can be converted into ATP). In the next step, succinate is converted into fumarate by succinate dehydrogenase and one molecule of $\mathrm{FADH}_{2}$ is produced. Fumarate undergoes a hydration reaction and L-malate is formed. Finally, malate is oxidized to oxaloacetate and a third molecule of NADH is formed. The regenerated oxaloacetate is now available to accept another molecule of acetyl-CoA for the initiation of a new cycle (Jeremy M Berg, John L Tymoczko, 2002) (Fig. I).

The electron transport chain (ETC) is coupled with the TCA cycle through the regeneration of $\mathrm{NAD}^{+}$from $\mathrm{NADH}$ that generates a proton gradient across the inner mitochondrial membrane, the gradient is used by the ATP-synthase to power ATP production. In the inner membrane of the mitochondria, five protein complexes take part in the ETC, of which the first four are involved in the generation of the proton gradient across the membrane. In fact, these complexes accept the electrons from $\mathrm{NADH}$ and $\mathrm{FADH}_{2}$, generated by glycolysis and TCA cycle, and transfer them to the ultimate acceptor of electrons, oxygen, to produce $\mathrm{H}_{2} \mathrm{O}$. This process is accompanied by the pumping of protons from the matrix of mitochondria into the intermembrane space. The electrochemical gradient that is generated by pumping the 
protons, provides the complex- $\mathrm{V}$ with the required energy for ATP synthesis. It is estimated that each molecule of $\mathrm{NADH}$ and $\mathrm{FADH}_{2}$ produces 2.5 and 1.5 molecules of ATP respectively. Therefore, it is estimated from one molecule of acetyl-CoA, 10 molecules of ATP are produced under aerobic conditions (Jeremy M Berg, John L Tymoczko, 2002).

\subsection{Fatty acid metabolism}

\subsubsection{Fatty acids}

FAs play an important role in energy homeostasis in the body during a metabolically challenging condition (Houten et al., 2016). These compounds are essential monomeric components of all different classes of lipids (including triacylglycerols (TAGs), phospholipids, sterol lipids, and sphingolipids). FAs are composed of a carbon chain that terminates with a carboxylic acid functional group (-COOH). They are very diverse in their carbon chain length, and the saturation status on their chain makes them even more diverse (Tracey et al., 2018). Based on the number of carbon atoms in their backbone FAs are classified into four groups although the definition for each group might differ among researchers: 1) short-chain FAs (SCFAs) composed by less than seven carbon atoms; 2) medium-chain FAs (MCFAs) with 7-12 carbon atoms in their structure (Schönfeld and Wojtczak, 2016); 3) long-chain FAs (LCFAs) with 12-20 carbons and 4) FA with more than 20 carbons in their backbone, considered as verylong-chain FAs (VLCFAs) (Kihara, 2012).

\subsubsection{Extracellular and intracellular sources of fatty acids}

It is postulated that the transport of FAs into the cells happens via two mechanisms: 1 ) passive diffusion (referred to as flip-flop model): a non-ATP consuming process whose efficiency depends on the size and lipophilicity of the FAs (e.g. the diffusion of SCFAs and MCFAs is easier than LCFAs) and 2) protein-mediated transport of FAs with high specificity for LCFAs and VLCFAs. There are known proteins involved in the latter process, including FA translocase (FAT/CD36) and plasma membrane FA-binding protein, or FABPpm (Houten et al., 2016), intracellular FA-binding proteins (FABP1-9), FA-transport proteins (FATP1-6) and 
caveolin1 (Mitchell et al., 2011). FAT/CD36 was discovered in 1993 as a protein involved in the transport of LCFAs in rat adipocytes (Abumrad et al., 1993). In 2004, Phol and colleagues reported disruption of lipid rafts by applying beta-cyclodextrin or overexpression of a dominant-negative mutant of caveolin-3, impairs uptake of VLCFAs into 3T3-L1 adipocytes (Pohl et al., 2004). In their following studies, the authors could show that FAT/CD36 specifically localized in lipid rafts and there was no synergistic effect between disruption of lipid rafts and FAT/CD36 inhibition on FA uptake. Based on these observations, a model describes how FAT/CD36, from intracellular nonlipid raft domains, can be recycled to the lipid raft microdomains in the plasma membrane. The process seemed to be involved in caveolin-1 function according to evidence shown in mouse embryonic fibroblasts, obtained later in 2006 by Ring (Pohl et al., 2005; Ring et al., 2006). FATP1-6 are bifunctional proteins that, besides their role in FA uptake, show acyl-CoA synthetase activity. FABP proteins are also classified into membrane-associated FABPs and cytosolic ones. The first group is not well understood and seems to be present on the extracellular surface of the plasma membrane as the treating of hepatocytes with trypsin shows to decrease the uptake of LCFAs (Stremmel et al., 1986). The cytosolic group of FABP proteins has been investigated more deeply: this family of proteins appears to be highly conserved among different species, from Drosophila melanogaster and Caenorhabditis elegans to mice and humans (Makowski and Hotamisligil, 2004). The first reported member of FABP proteins is FABP-1 (Liver- FABP), with a very high level of expression in tissues like liver, intestine, and kidney (2-5\% of cytosolic proteins) (Atshaves et al., 2010). FABPs reversibly bind to FAs and are highly expressed in cells that are active in uptaking and metabolizing FAs. Several roles for FABP have been reported, including: 1) increasing FA uptake by cells, 2) protecting cells from toxic effects of LCFAs (like detergent effect and inhibitory effect on enzymes), 3) enhancing intracellular transport of FAs and their distribution among different organelles (including mitochondria and peroxisomes for metabolism), 4) transport of LCFAs to the nucleus for interaction with nuclear receptors involved in metabolism, e.g. PPAR $\alpha$ (Makowski and Hotamisligil, 2004; Atshaves et al., 2010).

Although several proteins and cellular machinery involved in the uptake of FAs have been discovered, the exact mechanism of action of FAT/CD36 and other components of FA uptake are still under investigation. 
Besides the uptake of FAs from the extracellular space, other sources of FAs are present inside the cell: lipid droplets. These are intracellular organelles containing neutral FAs, sterol esters carrying a polar lipid monolayer on the surface, and proteins involved in the metabolism of FAs (Nishimoto and Tamori, 2017). These organelles are formed in the endoplasmic reticulum, where neutral lipids are synthesized. When needed, the FAs stored in lipid droplets and intracellular membranes are released as endogenous sources of FAs that can be mobilized for ATP production (Welte and Gould, 2017). The release of FAs from lipid droplets is performed either by lipid droplet associated lipases or by lipophagy, the process of autophagy of lipid droplets (Zechner et al., 2012). Autophagy is a process during which cytoplasmic materials are digested in lysosomes. This phenomenon is induced under metabolic stress such as starvation that results in increase in digestion of nonessential cellular materials to acquire energy metabolites for ATP production. There are three different types of autophagy: 1) chaperonemediated autophagy in which proteins are directly imported into lysosomes for degradation, 2) microautophagy in which cytoplasmic materials are engulfed by lysosomal membrane and are digested in the lysosome and 3) macroautophagy a process during which a double membrane is formed around the targets and autophagosome is formed that later upon fusion with lysosome the targeted materials are digested (Glick et al., 2010). Under feeding state, the mammalian target of rapamycin1 (mTORC1) is active and phosphorylates transcription factor EB (TFEB), a master regulator of autophagy. When TFEB is phosphorylated, its translocation into nucleus is inhibited. Under starvation conditions, mTORC1 is detached from the lysosomal membrane and is inactive, therefore, TFEB is dephosphorylated and is translocated into the nucleus, where it induces transcription of several genes involved in autophagy and lysosomal biogenesis (Sardiello et al., 2009; Settembre et al., 2011).

\subsection{3 $\beta$-Oxidation of fatty acids in mitochondria and peroxisomes}

Degradation of FAs in mitochondria is essential for the energy homeostasis of the cells, particularly during metabolic stress conditions, such as fasting and exercise (Chegary et al., 2009). Beta-oxidation starts with the activation of FAs, an esterification reaction in which coenzyme A (-COA) is added to free FA (FFA) and acyl-CoA is formed. Following activation, FAs 
can be transported into either mitochondria or peroxisomes for beta-oxidation (Houten et al., 2016; Tracey et al., 2018).

\subsubsection{Import of fatty acids into mitochondria}

Depending on their chain length, the mechanism of transport of FAs into mitochondria is different. Short and medium-chain FAs enter mitochondria by diffusion and are activated in the matrix of mitochondria. However, the mitochondrial membrane is impermeable to LCFAs (and acyl-CoA) and their transport into mitochondria is mediated by the carnitine shuttle (Wanders et al., 2010). The components of FA import into mitochondria are 1 ) carnitine ( $\beta$ hydroxy- $\gamma$-trimethylammonium butyrate) that is taken up into the cells by high-affinity organic cation transporter novel 2 (OCTN2), and 2) three proteins including carnitine palmitoyltransferase 1(CPT-1), carnitine acyl-carnitine translocase (CACT) and carnitine palmitoyltransferase 2 (CPT-2).

Carnitine palmitoyl-transferase 1 (CPT-1) is an integral membrane protein, located in the outer membrane of mitochondria and it is the only enzyme in the mitochondrial FA-oxidationsystem that exists in tissue-specific isoforms. This enzyme converts long chain acyl-CoA into long chain acyl-carnitine. Three isoforms for this enzyme have been reported, CPT1a and CPT1b which are called liver-type and muscle-type respectively, and CPT1c, another isoform reported to be neuron-specific. However, CPT1c enzymatic activity and its role in lipid metabolism are not clear yet (Wolfgang, 2016). In the next step of import, carnitine acyl-carnitine-translocase (CACT), an antiporter located in the inner mitochondria membrane, transfers one acyl-carnitine molecule into the matrix of mitochondria, in exchange with one molecule of carnitine. This antiporter is not solely specific to long-chain acyl-CoA and has been reported to be involved in the transport of short-chain acyl-carnitines, such as acetyl-carnitine and propionyl-carnitine produced in peroxisomes (Wanders and Waterham, 2006). CPT2 is a protein loosely associated with the inner face of the inner mitochondrial membrane, and it reconverts acyl-carnitine into acyl-CoA, a substrate for beta-oxidation enzymes (Swigoňová et al., 2009) (fig. I). 


\subsubsection{Mitochondrial $\beta$-Oxidation system}

$\beta$-Oxidation is a cyclic process that degrades FAs into acetyl-CoA units. The betaoxidation process can be divided into four steps: 1) dehydrogenation, 2) hydration, 3) dehydrogenation and 4) thiolytic cleavage.

Dehydrogenation of acyl-CoA molecules during the first step of beta-oxidation is performed by acyl-CoA dehydrogenase (ACAD). Three ACAD enzymes have been identified: based on their specificity for their substrates, are called short-chain acyl-CoA dehydrogenase (SCAD), medium-chain acyl-CoA dehydrogenase (MCAD) and long-chain acyl-CoA dehydrogenase (LCAD). All these enzymes use flavin adenine dinucleotide (FAD) as a coenzyme and upon their activity, FADH2 and trans-2-enoyl-CoA are formed (Swigoňová et al., 2009). Recently ACAD9, a new acyl-CoA dehydrogenase, has been reported. ACAD9 plays an important role in the assembly of complex I in mitochondria, along with its dehydrogenase activity on long-chain acyl-CoA (Zhang et al., 2002; He et al., 2007; Schiff et al., 2014).

In the second reaction of beta-oxidation, which is catalyzed by enoyl-CoA hydratases, one molecule of $\mathrm{H} 2 \mathrm{O}$ is added to the existing double bond in 2-enoyl-CoAand 3-hydroxyenoylCoA is formed. There are two proteins catalyzing this reaction: 1) long-chain enoyl-CoAs hydratase, as part of the mitochondrial trifunctional protein (MTP) which acts on long straightchain enoyl-CoAs, with the highest activity on C16:1 substrate and 2) crotonase that acts on short-chain enoyl-CoAs. enoyl-coAs with 4 to 16 carbon in their backbone, are substrates for crotonase. However, the activity of crotonase decreases with the increase in chain length of enoyl-CoAs. Since long chain enoyl-CoAs hydratase does not act on crotonyl-CoA it has been suggested that crotonase is needed at least for handling crotonyl-CoA.

The third step of beta-oxidation is the conversion of L-3-hydroxyenoyl-CoA to 3ketoacyl-CoA. This reaction is catalyzed by two 3-hydroxyacyl-CoA dehydrogenases: 1) longchain 3-hydroxyacyl-CoA dehydrogenase (LCHAD), that is part of MTP with its highest activity on C16 substrates and no activity on C4 substrates. 2) short-chain 3-hydroxyacyl-CoA dehydrogenase (SCHAD) which acts on substrates with 4 to 16 carbon in their backbone.

The last step of beta-oxidation is the cleavage of 3-ketoacyl-CoA and the formation of acetyl-CoA and shortened acyl-CoA. Three Ketoacyl-CoA thiolases have been identified in mitochondria, including 1) long chain 3-ketoacyl-CoA thiolase (LCKAT, part of MTP) that acts 
on substrates with 6 to 16 carbon atoms in their backbone, 2) medium chain 3-Ketoacyl-CoA thiolase (MCKAT), 3) beta-ketothiolase that uses 2-methylacetoacetyl-CoA as unique substrate and plays an indispensable role in isoleucine degradation (Wanders et al., 2010).

The shortened acyl-CoA produced at the end of each cycle of beta-oxidation could undergo a new cycle(s) of beta-oxidation till it is completely breakdowns into acetyl-CoA.

The end product of beta-oxidation, acetyl-CoA, and propionyl-CoA, could have different fates. acetyl-CoA can enter the TCA cycle for complete degradation into $\mathrm{CO} 2$ and production of ATP, NADH, and FADH2 as explained before in the glucose metabolism section. All FADH2 and $\mathrm{NADH}$, produced during beta-oxidation and complete degradation of acetyl-CoA in the TCA cycle, can transfer their electrons into the electron transport chain, for the generation of a proton gradient across the inner mitochondrial membrane, used for ATP production (Tracey et al., 2018).

The propionyl-CoA that is produced upon degradation of odd-numbered carbon chain molecules, can be carboxylated and converted into succinyl-CoA (intermediate of TCA cycle) via three reaction steps (Mazumder et al., 1961) (Fig. I).

\subsubsection{Peroxisomes}

For the first time, the term "Microbodies" was used in 1954 by Johannes Rhodin, to report the presence of single membrane cytoplasmic bodies observed on micrographs of convoluted tubule cells of the mouse kidney. Two years later, the presence of microbodies in rat liver tissue was reported by Rouiller and colleagues. (Rouiller and Bernhard, 1956). Later on, de Duve's group isolated microbodies from the rat liver, and by studying their biochemical properties, they found the presence of several enzymes involved in the production and degradation of hydrogen peroxide. These microbodies were termed "peroxisomes".(Gabaldón, 2010; De Duve, 1996). Soon after, a specific cytochemical staining for the detection of peroxisomes with light and electron microscopy was developed with the introduction of the alkaline 3, 3'diaminobenzidine (DAB) reaction for catalase (Novikoff and Goldfischer, 1969).). Since then, the presence of these organelles was reported in other species and, like mitochondria, peroxisomes were recognized as indispensable, ubiquitous eukaryotic organelles (Schrader 
and Fahimi, 2008a). Further studies showed substantial differences in peroxisomes between different organisms and even within the same organism, peroxisome's properties can differ between organs (Titorenko, 2001). In mammals, peroxisomes play an important role in cellular lipid metabolism including 1) FA beta-oxidation, 2) ether phospholipid (plasmalogen) biosynthesis, 3) FA alpha-oxidation 4) biosynthesis of cholesterol, 5) bile acids, 6) prostaglandins (Titorenko, 2001). as well as 7) cellular redox balance (Schrader and Fahimi, 2008a). In terms of their size, peroxisomes also show differences that range from $0.1-1.0 \mu \mathrm{m}$ (Schrader and Fahimi, 2008b).

\subsubsection{Peroxisomal beta-oxidation}

The degradation of more complex FAs takes place in peroxisomes. Since very long-chain acyl-CoA(s) are not substrates of the carnitine shuttle in the membrane of mitochondria, this group of FAs cannot enter these organelles and their degradation is limited to peroxisomes (Longo et al., 2016). In mammals, three ATP- binding cassette (ABC) transporters (subfamily D) in the membrane of peroxisomes are responsible for the import of FAs into peroxisomes. All three transporters use acyl-CoA as substrate and, during the transport, remove CoA and release free FAs into the lumen of the peroxisome. Acyl-CoA synthases interacting with transporters regenerate acyl-CoA (Longo et al., 2016; Theodoulou et al., 2016). Acyl-CoA undergo betaoxidation inside peroxisomes: this process is basically similar to beta-oxidation in mitochondria and consists of four reactions, including dehydrogenation, hydration, dehydrogenation, and thiolytic cleavage. However, the enzymes that catalyze the reactions are different. The first step is catalyzed by acyl-CoA oxidase (ACOX) instead of acyl-CoA dehydrogenase in mitochondria and the removed hydrogens are used for the formation of $\mathrm{H}_{2} \mathrm{O}_{2}$, subsequently converted to $\mathrm{H}_{2} \mathrm{O}$ by catalase. ACOX1 (isoform a and b), ACOX2 and ACOX3 catalyze this step. Steps two and three are catalyzed by multifunctional proteins, MFP1 and MFP2. The activity of MFP1 results in products with L- configuration while MFP2 generates end products with Dconfiguration. The formed trans-2-enoyl-CoA in step one is hydrated by MFP1 and MFP2 and 3-hydroxy-acyl-CoA is produced. This is a substrate for the 3-hydroxy-acyl-CoA dehydrogenase domain of MFP that leads to the generation of 3-ketoacyl-CoA. The formation 
of 3-ketoacyl-CoA during the third step results in the formation of one molecule of NADH. In the last step, Ketoacyl-CoA thiolase removes acetyl-CoA from 3-Ketoacyl-CoA and acetyl-CoA and shortened acyl-CoA are formed (Baes and Van Veldhoven, 2012). The end products of beta-oxidation in peroxisomes are acetyl-CoA, propionyl-CoA and shortened acyl-CoA that could be converted to carnitine esters. Two enzymes are involved in this conversion: carnitineoctanoyl transferase (CROT) and carnitine acetyltransferase (CRAT) that seem to be involved in the export of beta-oxidation products from peroxisomes (Baes and Van Veldhoven, 2012; Westin et al., 2008).The machinery involved in export of FAs from peroxisomes remains largely unknown, however, OCTN3 might be a promising candidate as it is a carnitine transporter localized in the peroxisomal membrane (Lamhonwah et al., 2005). It has also been suggested to be involved in export of end products of beta oxidation (acyl-carnitine) from peroxisomes (Januszewicz et al., 2009).

\subsubsection{Interaction between peroxisomes and mitochondria}

The complete degradation of peroxisomal beta-oxidation products for the generation of ATP depends on their interaction with mitochondria. Although the peroxisomal beta-oxidation is very similar to the mitochondrial one, the purpose of this process in peroxisomes is the shortening of FA chain length (Baes and Van Veldhoven, 2012; Longo et al., 2016; Tracey et al., 2018). For example, rat liver peroxisomes do not use butyryl-CoA as substrate and their activity is less with octanoyl-CoA than with lauroyl-CoA or palmitoyl-CoA (Lazarow, 1978). In addition, degradation of VLCFAs is restricted to peroxisomes and only after shortening these can be a substrate for mitochondrial beta-oxidation (Violante et al., 2013). Moreover, since peroxisomes do not carry the enzymatic machinery for the TCA cycle and the ETC, the complete degradation of FA to $\mathrm{CO}_{2}$ and ATP never takes place inside this organelle. Therefore, transfer of end products of peroxisomal beta-oxidation including acetyl-CoA, propionyl-CoA, and shortened FAs into mitochondria for ATP production is necessary (Longo et al., 2016; Tracey et al., 2018),(fig. I). 


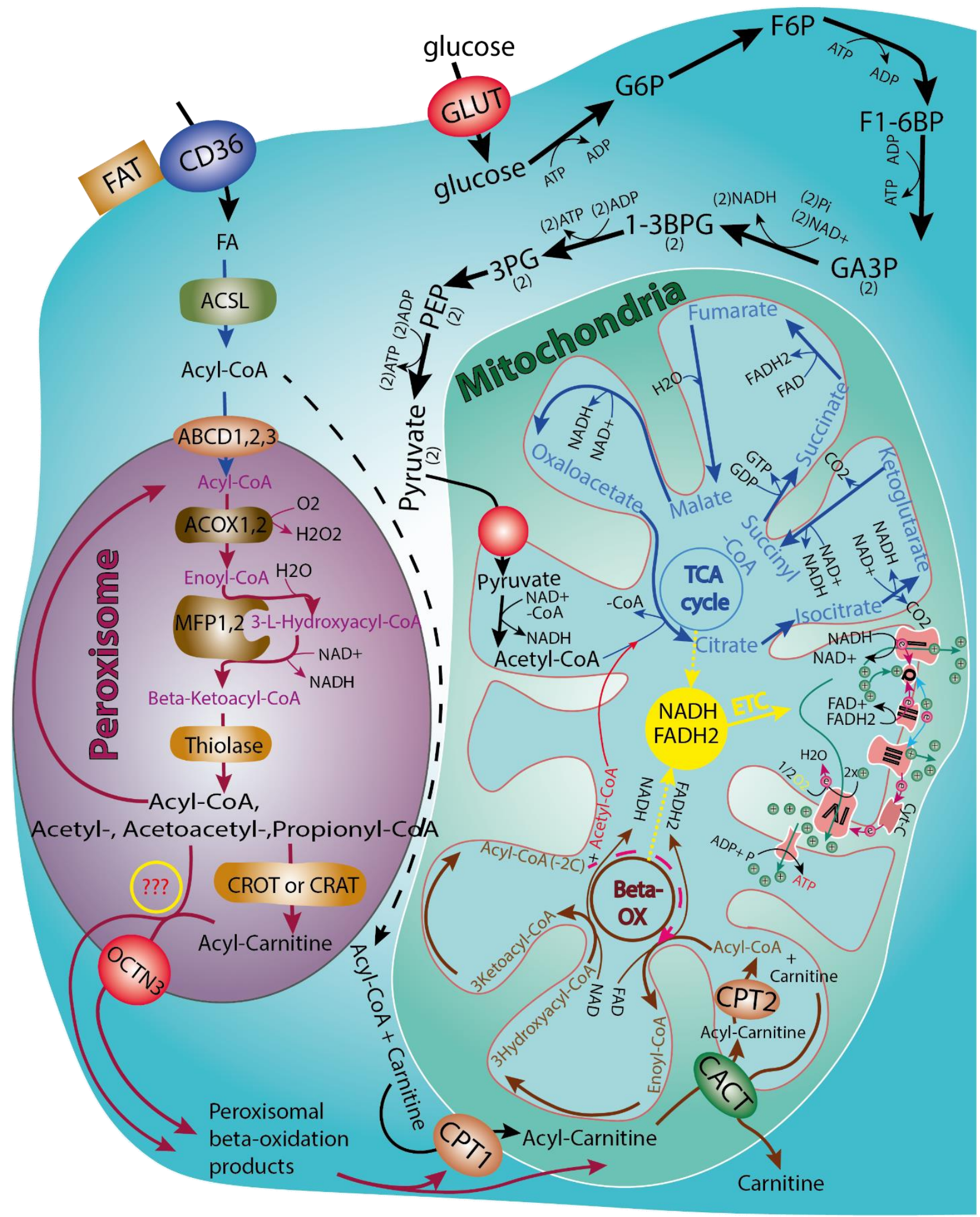

figure I. Glucose metabolism and interaction between peroxisomal and mitochondrial beta-oxidation. Glucose enter the cells via GLUT transporters and upon glycolysis is converted into pyruvate (upper right corner). Inside the mitochondria, pyruvate is converted to acetyl-CoA that enters the TCA cycle (blue arrows) for further degradation and ATP production. FAs, as another source of energy for the cells, can be released from endogenous sources (like 
lipid droplets and intracellular membranes) or they can be up taken by cells by FAT/CD36 proteins present in the plasma membrane. After activation in the cytoplasm (conversion to acyl-CoA), these precursors enter mitochondria or peroxisomes for beta oxidation. Entry of SCFAs and MCFAs into mitochondria is performed by diffusion, however, import of LCFAs is dependent on carnitine/acyl-carnitine transport system (since VLCFAs are not substrate for CPT1, degradation of VLCFAs do not occur in mitochondria). When in the matrix of mitochondria, FA can undergo beta-oxidation (brown arrows, lower right corner) that results in the production of FADH2, NADH, and the formation of acetyl-CoA and shorter chain acyl-CoAs (-2C). The produced acetyl-CoA during beta oxidation can enter the TCA cycle for complete degradation. Degradation of FAstake place in peroxisomes (in purple) as well and degradation of VLCFAs is restricted to peroxisomes. Since peroxisomes are devoid of TCA cycle and ETC, after beta-oxidation in peroxisome, the chain shortened FAs, acetyl-CoA and other end products should enter mitochondria for ATP production (unknown mechanisms: proteins like CROT, CRAT and OCTN3 might involve in this process). Therefore, it is generally accepted that the interaction between these two organelles is necessary for ATP production. The produced FADH2 and NADH in TCA cycle or beta-oxidation are used by the ETC in the inner mitochondrial membrane to establish a proton gradient that is necessary for ATP generation during OXPHOS.

\subsection{Metabolism in WM}

\subsubsection{WM energy demands - glucose metabolism}

The structure and the organization of white matter (WM) are different than those of gray matter (GM), therefore the energy demands and regulation of metabolism are different too. In WM tracts, the dendrite of neurons that form synapses (axon-axonal synapses) for signal processing are not densely present and only recently it has been reported that axonoligodendrocyte precursor cell (OPC) synapses are present during development and may also exist after myelination is complete (Kukley et al., 2007; Ziskin et al., 2007). However, it is not known whether the amount of energy that is required by an axon-OPC synapse in WM is comparable with axon-axon synapse of gray (Harris and Attwell, 2012).

The absence of dendrites and therefore the lack of the most energy-consuming part of a neuron reduces the energy demand of WM significantly (Sokoloff and Clarke, 1989). Despite the lower energy demand of WM, even under physiological conditions, processes like regeneration of resting potential along the axons, transport of organelles and proteins to the synaptic terminal as well as the maintenance of housekeeping functions, pose a metabolic challenge for axons and glial cells of WM (Harris and Attwell, 2012; Baltan, 2014). In addition, two WM structural features make energy metabolism even more complex: 1) the vascular network in WM is less dense than gray matter, reducing blood supply in this region (Moody et al., 1990); 2) the presence of myelin, a multilayer structure of phospholipids that is formed by consecutive wrapping of oligodendrocyte's membrane around the axons, which on one side 
increases the resistance (and lower the capacitance) of the axonal membrane but at the same time, it restricts the axons to have access to extracellular metabolites, with the exception of the node of Ranvier (Nave, 2010).

In 1994 Pellerin \& Magistretti described the details of a model through which astrocytes support neurons, astrocyte-neuron lactate shuttle hypothesis (ANLSH). Based on this model, after release of glutamate from presynaptic neurons, the nearby astrocyte processes start removing glutamate via sodium co-transporters that results in an increase in intracellular $\mathrm{Na}+$ concentration in astrocytes and activation of $\mathrm{Na} / \mathrm{K}$ ATPase pumps. The sudden increase in the ATP demand of astrocytes lead to increase in uptake of glucose from vasculature that after conversion into lactate are shuttled to neurons (Pellerin and Magistretti, 1994). The most parts of the machinery and required structures for the astrocyte-neuron lactate shuttle transfer system are present in WM and increasing evidence supports the hypothesis of astrocytic metabolic support of axonal function (Lee et al., 2012; Hirrlinger and Nave, 2014a; Fünfschilling et al., 2012; Saab et al., 2016; Trevisiol et al., 2017). It has been reported that astrocytes with their end-feet in proximity to vasculature, are present at the nodal region in the central nervous system and these perinodal astrocytes can even form junctions with the axonal membrane trough glycoproteins involved in cell-cell interaction, like J1, N-CAM, cytotactin and extracellular matrix components produced by glial cells (Black and Waxman, 1988). The expression pattern of monocarboxylate transports, MCTs, has also shown that MCT1, expressed in lactate producing tissues is expressed by astrocytes and MCT2 that seems to be more involved in the uptake of lactate, is present in the axonal membrane (POOLE et al., 1996; Bröer et al., 1997). The Lactate dehydrogenase isozymes, an enzyme that converts lactate to pyruvate and vice-versa, LDH5 and LDH1 show also a specific expression pattern: LDH5, that favors more the conversion of pyruvate to lactate, is expressed in astrocytes and LDH1, that rather converts lactate to pyruvate (that feeds the TCA cycle) is abundant in the axonal compartment (Bittar et al., 1996). These studies have been supported by functional observations obtained from electrophysiology ex-vivo measurements in the optic nerve (used as a model for a WM tract (Stys et al., 1991), where the conduction of all firing axons is presented as compound action potential (CAP). In the optic nerve, the majority of axons are myelinated, and astrocytes are the only cells that contain glycogen as the storage form of glucose. When glucose is removed from the solution (aCSF, glucose deprivation) the nerve can still respond to the stimulation and maintain its 
conduction for as long as $20 \mathrm{~min}$, by degrading the stored glycogen in astrocytes. Astrocytes under glucose deprivation can, in fact, produce and release lactate that is uptaken by the axon via MCT2 for ATP production (Brown et al., 2004). Furthermore, it has been observed that lactate, as the end product of glycolysis, is capable of maintaining the nerve function as good as glucose, indicating that lactate can be used by axonal mitochondria for ATP production (Brown et al., 2003).

\subsubsection{Effect of myelination on signal propagation and energy utilization in WM}

In the central nervous system, oligodendrocytes form the myelin sheath around the axon. The term 'myelin' was coined for the first time by Rudolf Virchow in 1854 under the assumption that myelin was made by neuronal secretions. Later on, in 1921, by the advance in histological techniques, Pio del Rio-Hortega re-discovered oligodendrocytes (after Robertson in 1899) and called them oligodendroglia. In 1924 Penfield, jointly with del Rio-Hortega, hypothesized that myelin could be made by oligodendrocytes. Finally, in 1954 Geren could show that Schwann cells form myelin in the peripheral nervous system. Following on this observation, in 1962, Bunges could prove that myelin in the CNS is formed by oligodendrocytes. Each oligodendrocyte in the CNS can make contact with 20 to 60 points on the axons where they form myelin segments stretching for $\sim 20$ to $200 \mu \mathrm{m}$ longitudinally. These stretches of myelin are separated by nodes of Ranvier (Simons and Nave, 2016).

The best-known function of myelin is the increase in the speed of action potential (AP) propagation along the axon. The electrical properties of axons are determined by the presence of a negative electric potential across the plasma membrane due to a separation of charges, which is in turn created by the different concentrations of ions $\left(\mathrm{K}^{+}, \mathrm{Na}^{+}, \mathrm{Cl}^{-}\right.$and $\left.\mathrm{Ca}^{2+}\right)$ across the cell membrane. The membrane potential of all cells is reported to be typically between -30 to $-70 \mathrm{mV}$. This potential does not change with time in most mammalian cell types, with the exception for neurons and muscle cells that are electrically active. Under resting conditions, there is a potential across the axonal membrane of around $-70 \mathrm{mV}$. This negative potential is the consequence of an electrochemical gradient that is produced by the $\mathrm{Na}^{+} / \mathrm{K}^{+} \mathrm{ATPase}$ pumps that distribute $\mathrm{K}^{+}$and $\mathrm{Na}^{+}$ions differently across the membrane so that the $\mathrm{K}^{+}$concentration is higher inside the cells and the $\mathrm{Na}^{+}$concentration is higher in the extracellular space. At the 
value of resting membrane potential, the voltage-gated ion channels are closed, but when this value changes towards more positive values (approaching the threshold), the depolarization can trigger an AP that involves the activity of $\mathrm{Na}^{+}$and $\mathrm{K}^{+}$voltage-gated channels. The AP has three phases including depolarization, hyperpolarization and return to the resting potential. In the first phase, the membrane potential in a region of the neuron (e.g. dendrite or axon hillock) depolarizes slightly (due to activation of postsynaptic ionotropic receptors or in response to integration of EPSP). By reaching the threshold of stimulation, the voltage-dependent $\mathrm{Na}^{+}$ channels open for a short period of time resulting in an influx of $\mathrm{Na}^{+}$ions and depolarization associated with the rise of an AP. Because the voltage-gated $\mathrm{K}^{+}$channels open at more positive values of $R_{m}$ and with sub-millisecond delay after the initial depolarization, this kind of voltagegated $\mathrm{K}^{+}$channels are also called delayed $\mathrm{K}^{+}$channels and are responsible for the hyperpolarization phase, or second phase of the AP. Eventually, all the voltage-gated channels are closed and only non-voltage-gated $\mathrm{K}^{+}$channels that generate the negative potential of the membrane open (rectifying) and the potential of the membrane reaches the resting state after a short hyperpolarization phase. At the peak of the AP, the membrane depolarization is sufficient to passively spread downstream of the segment of membrane involved in the AP producing a propagation wave of the AP. The upstream propagation is prevented by the refractory period of the membrane (off-state of the ion channels). Although the activity of the $\mathrm{Na}^{+} / \mathrm{K}^{+}$ATPase does not directly play a role in the AP generation, and the nerves can normally fire thousands of time in the absence of ATP (since the ion flow across the membrane during each discharge causes only a minute change in concentration of $\mathrm{Na}^{+}$and $\mathrm{K}^{+}$ions), the continuous generation of AP requires the activity of the $\mathrm{Na}^{+} / \mathrm{K}^{+}$ATPase to restore the gradient of ions across the membrane (Lodish H, Berk A, Zipursky SL, 2000). This process has a cost in ATP molecules and is by far the most energy-consuming feature in the axon (Harris and Attwell, 2012). The alternation of myelinated vs. naked axon segments results in the propagation of AP from one node to the next is referred to as salutatory propagation, as opposed to the wave propagation and consequently, increases the speed of signal propagation along the axons (Hirrlinger and Nave, 2014a).

Myelination affects not only the velocity of signal propagation along the axon, but also affects energy metabolism in WM. In myelinated axons myelin sheaths cover most parts of the 
axons except for the nodes of Ranvier, therefore a large percentage of $\mathrm{Na}^{+}$channels are not involved in the influx of $\mathrm{Na}^{+}$during AP propagation. Therefore, based on our current understanding and available models, the consequence of minimizing the $\mathrm{Na}^{+}$influx is saving energy (Waxman, 1997; Hartline and Colman, 2007; Harris and Attwell, 2012). However, the conclusion on a putative energy-saving feature conferred by myelin of WM tracts is still under debate, given that the amount of energy needed for maintaining the myelin structure itself is not clear yet.

Besides the clear advantage of myelination in speeding AP propagation, covering the axons with myelin sheath makes also an effective barrier on the surface of the axon and limits the accessibility of myelinated segments to the extracellular space and its metabolites (Hirrlinger and Nave, 2014b). In order to overcome this limitation introduced by compacted multilayer structure with low turnover, it is hypothesized that myelin has developed to support the ensheathed axon by directly providing metabolites (Nave, 2010). Therefore, it seems that myelin is not a merely passive structure but increasing evidences suggests that myelin rather supports axonal function by providing metabolites to the axons and plays an important role in long term integrity of axons in WM. Oligodendrocytes that strongly express MCT1 release lactate into the peri-axonal space (space between the axon and the oligodendrocyte's adaxonal membrane) and support axonal function. Impairment in this support mechanism can affect the axonal function and integrity (Fünfschilling et al., 2012) as it has been reported in cases of MCT1 downregulation, both in patients with amyotrophic lateral sclerosis (ALS) and in the murine model of the disease (MCT1 heterozygous mice) that resembles ALS symptoms and phenotype (Lee et al., 2012).This metabolic support by the oligodendrocyte needs to be finely tuned and adjusted on the energy demands of the axon. This is achieved by a constant communication between the axon and the oligodendrocyte that ensures that the axon receives enough substrates under constant activity (Saab et al., 2016). It has been shown that neurons form synapses with oligodendrocytes (referred to as Axo-Myelinic synapse) by releasing neurotransmitters along their axons and into the peri-axonal space (Douglas Fields, 2015). The axo-myelinic synapse activity correlates with neuronal activity. In 2016 Saab et al., using conditional knock-out mice lacking NMDA receptor in their oligodendrocytes, showed the coupling between release of NMDA from axons into the peri-axonal space and increase in 
glucose uptake by oligodendrocytes through translocation into the cell membrane of GLUT1, the main glucose transporter in oligodendrocytes (Saab et al., 2016).

\subsubsection{WM energy demands- fatty acid metabolism}

The brain is able to use FAs and its derivatives as a glucose-alternative fuel supply. Studies that used ${ }^{13} \mathrm{C}$ labeled octanoate, estimated that around $20 \%$ of the energy in rats' brain is coming from FAs under resting conditions (Ebert et al., 2003). Under specific conditions, when glucose is not enough to support brain metabolism, the role of FA catabolism becomes even more important and utilization of FAs and ketone bodies (as lipid derivatives) increases (Owen et al., 1967; Cahill, 2006; Klosinski et al., 2015). The fact that the brain can use FAs and its derivatives as fuel is well established (Ebert et al., 2003; Vignais et al., 1958).However, which cell types are involved in FA oxidation and under which condition FAs are used remains unclear. Studies on neuron, astrocyte and oligodendrocyte cultures from developing rat brain (1-2 days old) have shown that all three cell types are able to oxidize ketone bodies, while astrocytes were found to be the only cell type capable of oxidizing FAs to $\mathrm{CO}_{2}$ (Edmond et al., 1987; Auestad et al., 1991). These observations suggest that mitochondria in astrocytes are able to oxidase FAs during the suckling period. However, it remains unclear whether these observations can be extrapolated to mature brain cells.

Peroxisomal beta-oxidation in myelin plays an important role in the health of the brain. The importance of peroxisomes in the brain has been reflected in neurodegenerative peroxisomal disorders. In several years, using genetic tools and generating mouse conditional knock-out, peroxisomes have been inactivated in a cell-specific manner. One of the targeted proteins for studying the role of peroxisomes is PEX5. PEX5 is part of the protein import machinery of peroxisomes that binds to the PTS1 signal sequence of peroxisomal proteins and targets them to the peroxisome. When PEX5 is knocked-out, the formed peroxisomes are lacking proteins in their lumen and thus are inactive. Inactivating peroxisomes in forebrain projection neurons (Pex $5^{f / f f} *$ Nex-Cre mice) does not cause any phenotype in mice probably because of the rare abundance of this organelle in neurons. When peroxisomes are inactivated in astrocytes (Gfap-Pex5 $5^{-/-}$mice) the accumulation of neutral lipids is observed in astrocytes 
and ependymal cells. However, this does not affect neurological function as the mice do not show any phenotype (Bottelbergs et al., 2010). Interestingly, the consequence of peroxisome

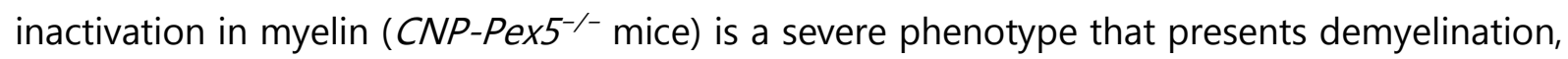
axonal loss (without neuronal death) and neuroinflammation (Kassmann et al., 2007; Baes and Van Veldhoven, 2012b). Ultrastructural studies of CNP-Pex5 ${ }^{-/-}$mice revealed an apparently normal myelin but presence of axonal swelling even before the onset of demyelination, implying that peroxisomes do not play an important role in myelination but their function is important for long term integrity of the axons that are far away from the soma of neurons (Kassmann et al., 2007). In addition, subcellular localization studies of peroxisomes have shown that the majority of peroxisomes are at the soma. However, peroxisomes can be present at the innermost and outermost layers of myelin and are accumulated in the paranodal region (Kassmann, 2014). These two observations along with 1) axonal swelling being a sign of energy deficit in axons (Ferreirinha et al., 2004) and 2) the presence of peroxisomes at the axo-glia interaction site (i.e. paranodes), has driven the authors to postulate an interaction between myelin-associated peroxisomes and moving mitochondria along the axons (Kassmann, 2014). Since in PEX5 mutants the cells contain inactive peroxisomes (Ghost peroxisomes), all the functions related to peroxisomal enzymes have been perturbed. Following studies that perturbed beta-oxidation in peroxisomes (e.g. by inactivating MFP2 in neural cells) showed that beta-oxidation plays an important role in peroxisomal disorders (Verheijden et al., 2013). Although these observations support the importance of peroxisomal beta-oxidation in myelin, there is no direct evidence showing the actual purpose of their function but speculations about their possible involvement in the turnover of membrane-associated FAs or, direct involvement in brain energy metabolism (Kassmann, 2014).

There is evidence suggesting that endogenous brain lipids could serve as a source of energy. In this respect, myelin represents a highly FA-riched structure, formed by the layering of the plasma membrane of oligodendrocytes (in the CNS) around the axons. Myelin similarly to the membrane of other cells, is composed of proteins and lipids. However, myelin has uniquely high lipid content: around $70 \%$ of the dry-weight of myelin is composed of lipids (Chrast et al., 2011; Ozgen et al., 2016). There are some observations suggesting that myelin is degraded when glucose is not sufficiently abundant to meet the energy demand of the brain. A decline 
in glucose metabolism has been reported in the brain of aged, schizophrenic and early stages of Alzheimer patients (de Leon et al., 1983; Mosconi, 2005; Yao et al., 2009; Chrast et al., 2011; Yang et al., 2017). This decline in glucose metabolism is followed by a shift to the utilization of FAs and ketone bodies as alternative fuels for the brain (Yao et al., 2010; Ding et al., 2013). Furthermore, this shift in metabolism is accompanied by changes in WM and myelin breakdown (Zhang et al., 2007; Lebel et al., 2012). Although these observations suggest that myelin-associated FAs are a source of energy under pathological conditions (Yao et al., 2011; Klosinski et al., 2015), their role in non-pathological conditions is understudied and poorly understood. 


\section{Objectives}

Although it has been shown that FAs can be metabolized by the brain, the importance of FA catabolism has been neglected to some extent as glucose has been regarded as the major source of energy in the brain. In this project we aimed for answering following basic questions related to the role of myelin and beta-oxidation of FAs in supporting energy demand of neurons, a subject with potential implication in brain diseases:

1) Glycogen, the storage form of glucose, is present in astrocytes to support brain function in time of need. In WM, oligodendrocytes wrap around the axons and form a multilayer structure enriched in FA called myelin. The best-known function of myelin is increasing the rate of signal propagation along the axon; however, it remains unclear whether myelin needs all its layers to exert is function or if it could still function normally by losing some of it layers as a source of energy. It therefore raises the question whether myelin is a storage form of FA that serves as a buffer of energy for the brain.

2) Despite reported studies showing FAs are metabolized by the brain, it has not been shown if FAs could support nerve function. To address this question using ex-vivo electrophysiology of the optic nerve, we could show if beta-oxidation of FAs is involved in supporting nerve function.

3) Even though, the importance of peroxisome in brain function has been clearly reflected in several diseases with impaired peroxisomal functions, the role of peroxisomes in supporting neuronal function has not been addressed. 


\section{Results}

\subsection{Results outlook:}

The results obtained from this project are explained in four sections that are closely related. In section one, the data regarding the evaluation of the mouse optic nerve (MON) as our model system and the effect of starvation on the viability of its different cell types will be reported. Section two includes the data suggesting that myelin-derived FAs are involved in supporting the survival of MON cells under starvation condition. In section three, we focus on the data supporting the role of FAs in nerve function. The acquired results from ex vivo electrophysiology combined with live imaging of $\mathrm{MON}$ and the effect of beta-oxidation inhibitors on the nerve function are reported in this section. The last section describes the obtained data from an in vivo mouse model for oligodendrocyte starvation, the GLUT1 ciKO mouse line, that shows how myelin is progressively degraded when glucose uptake is perturbed specifically in mature oligodendrocytes.

\subsection{Starvation effect on viability of mouse optic nerve cells}

\subsubsection{Evaluation of the mouse optic nerve as a model system}

The mouse optic nerve (MON) is a suitable model for studying metabolism in WM. In the optic nerve, all axons are appropriately aligned and myelinated by oligodendrocytes. Therefore, it is easy to prepare longitudinal and cross-sections for histological studies and it is possible to use them for studying the function of the nerve by doing ex vivo electrophysiology.

To evaluate the proper preparation of the nerve for histological studies, the optic nerves from wild type (WT) mice were incubated in aCSF containing $10 \mathrm{mM}$ glucose $(\mathrm{aCSF}+10 \mathrm{mM}$ glucose) for $16 \mathrm{~h}$ and $24 \mathrm{~h}$ and were stained with presidium iodide (PI) during incubation. PI is an impermeable dye that does not penetrate the cells with an intact plasma membrane and it only stains the nucleus of dead cells by intercalation into the DNA structure making it useful for detecting dead cells (Crowley et al., 2016). The obtained images from a longitudinal section of the incubated nerves revealed that the majority of cells are alive in the optic nerve and only some dead cells are usually observable at the nerve periphery and at the cutting sites of the 
nerve (fig. $1 A$ and B). Thank to Dr. Aiman Saab, Jennifer Günther and Annette Fahrenholz for teaching me the histology techniques.

A

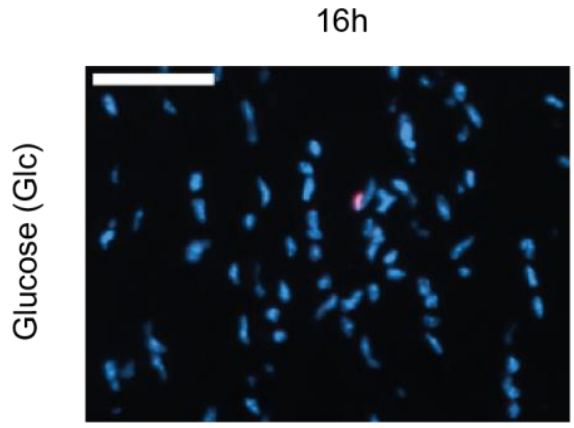

$24 \mathrm{~h}$

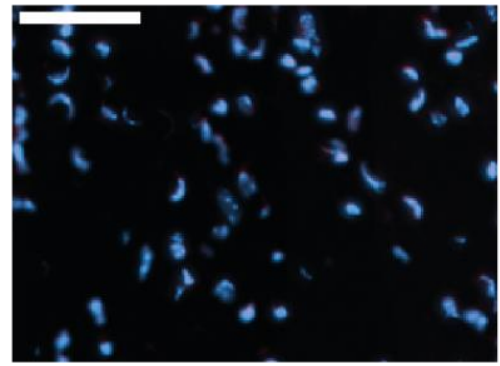

B

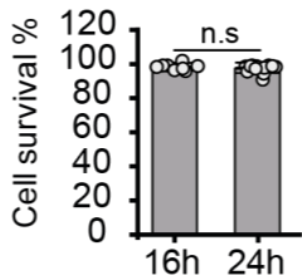

Figure 1. Evaluation of the optic nerve preparation for incubation purpose.

(A) The images from longitudinal optic nerve sections (8-micron thick) of wild type mice after $16 \mathrm{~h}$ (left) and $24 \mathrm{~h}$ (right) incubation in aCSF $+10 \mathrm{mM}$ glucose. The blue dots showing the nucleus of the cells were stained with DAPI and the red signal is PI that has been used for detecting the dead cells (Scale bar: $50 \mu \mathrm{m}$, frame Size: $200 * 150 \mu \mathrm{m}$ ). (B) Showing the quantified cells death in the whole longitudinal section from the optic nerve expressed in percent (ratio of PI / DAPI) after 16h and 24h. (Wild type mice, 8-9 weeks old, male or female, statistics: t-test, error bars: mean+/-SEM, $n \geq 8)$.

\subsubsection{Effect of starvation on viability of optic nerve cells}

In order to evaluate the susceptibility of the optic nerve to starvation condition, MON from WT mice were incubated for different time points, $16 \mathrm{~h}$ and $24 \mathrm{~h}$, in artificial cerebrospinal fluid (aCSF) containing $10 \mathrm{mM}$ sucrose (aCSF+10 mM sucrose) instead of glucose. For the sake of simplicity, hereafter Glucose condition (Glc) refers to aCSF+10 mM glucose and starvation condition (Stv) refers to aCSF+10 mM sucrose for all the incubation experiments, unless otherwise stated. Our data shows that $16 \mathrm{~h}$-starvation does not affect the viability of optic nerve cells. However, $24 \mathrm{~h}$ starvation induces death of $18.3 \%$ of optic nerve cells as reflected in the number of PI positive cells (Fig. 2A and B). These observations suggest that most of the cells in the optic nerve can tolerate starvation condition and raises the question of which cell types are dying. 
A

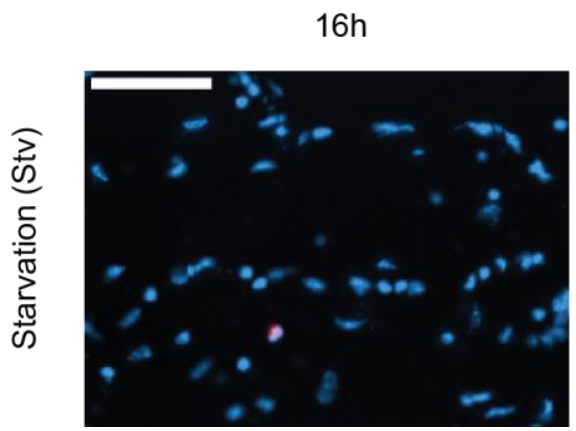

$24 \mathrm{~h}$

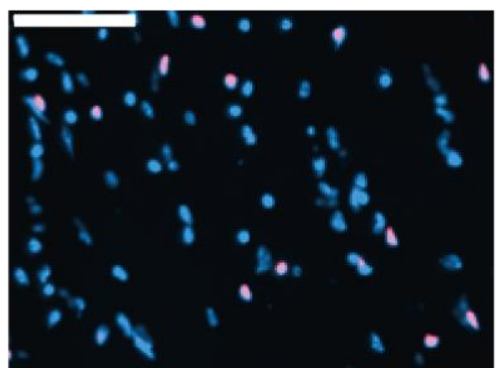

B

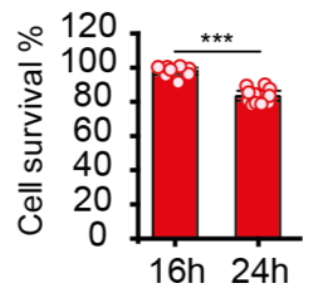

Figure 2. Effects of 16 and $24 \mathrm{~h}$ starvation on viability of optic nerve cells.

(A) The images from longitudinal optic nerve sections ( 8 microm thick) of wild type mice after $16 \mathrm{~h}$ (left) and $24 \mathrm{~h}$ (right) incubation in aCSF $+10 \mathrm{mM}$ sucrose (Stv). The blue dots showing the nucleus of the cells were stained with DAPI and the red signal is PI that has been used for detecting the dead cells during starvation (Scale bar: $50 \mu \mathrm{m}$, frame Size: $200 * 150 \mu \mathrm{m}$ ). (B) Is showing the quantified cells death in the whole longitudinal section from the optic nerve expressed in percent (ratio of PI / DAPI) after 16h and 24h starvation. (Wild type mice, 8-9 weeks old, male or female, statistics: t-test, error bars: mean+/-SEM, $n \geq 8$ ).

\subsubsection{Effect of starvation on viability of different cell types in the optic nerve}

In order to define which cell types are dying during starvation, the optic nerves from mouse lines expressing GFP or YFP in a cell-type specific manner were used. Since cell death is induced in the $24 \mathrm{~h}$-starved nerve (Fig. $2 \mathrm{~A}, \mathrm{~B}$ ), the nerves were incubated in aCSF $+10 \mathrm{mM}$ glucose/ or sucrose for $24 \mathrm{~h}$. After staining the nerves with PI, they were fixed and subjected to microscopy. The obtained data revealed that the frequency of oligodendrocytes, oligodendrocytes precursor cells (OPCs), microglia and astrocytes in incubated optic nerve for $24 \mathrm{~h}$ under normal condition are around $53 \%, 5 \%, 6 \%$ and $23.5 \%$, respectively and starvation has no significant effect on the population of the first three cell types. However, the number of quantified astrocytes has decreased to $10.61 \%$ under starvation conditions, accounting for death of $55 \%$ of astrocytes (Fig. $3 \mathrm{~A}$ and B). Adding to this $55 \%$ of dead astrocytes the percentage of astrocytes that are still showing a weak green signal and are PI-positive result in final calculation of survival of only around $30 \%$ of the astrocyte population after $24 \mathrm{~h}$ starvation (Fig. 3C). 
A
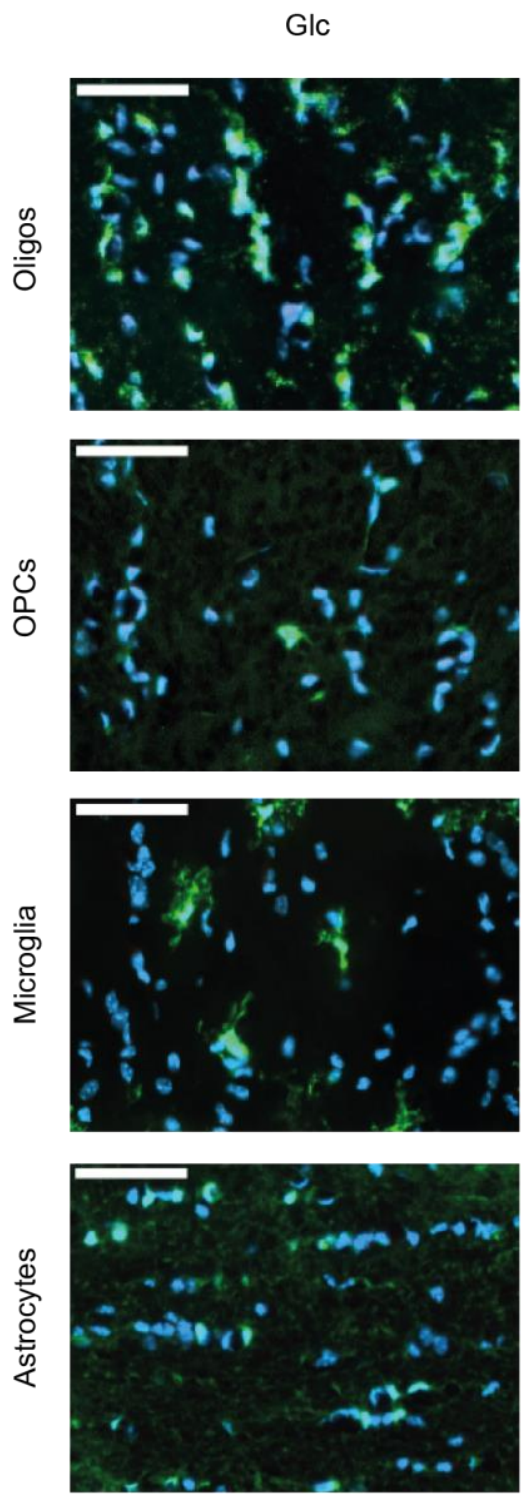

Stv
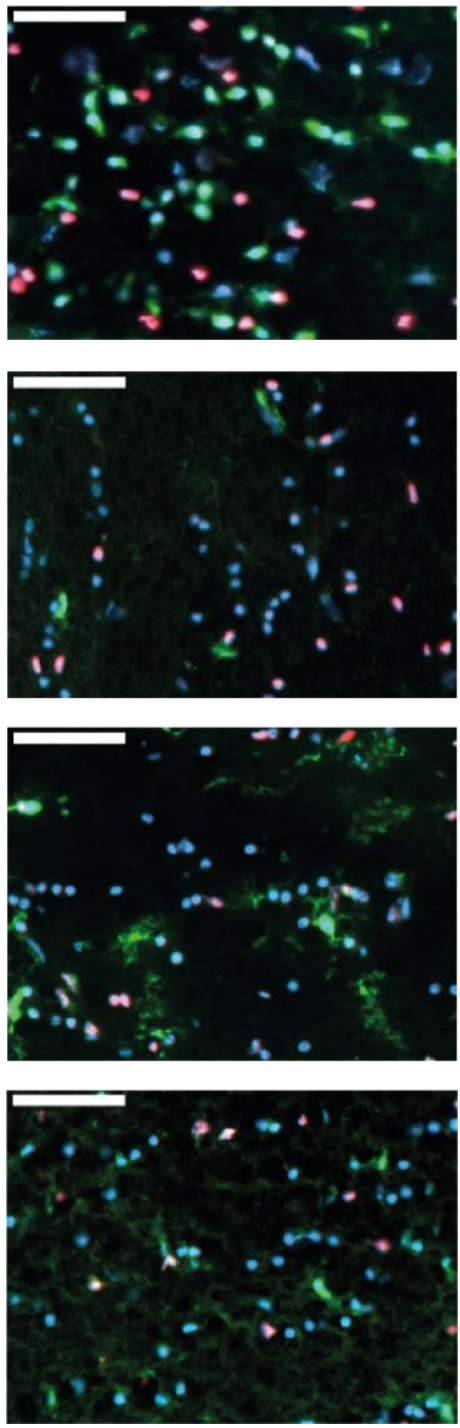

B

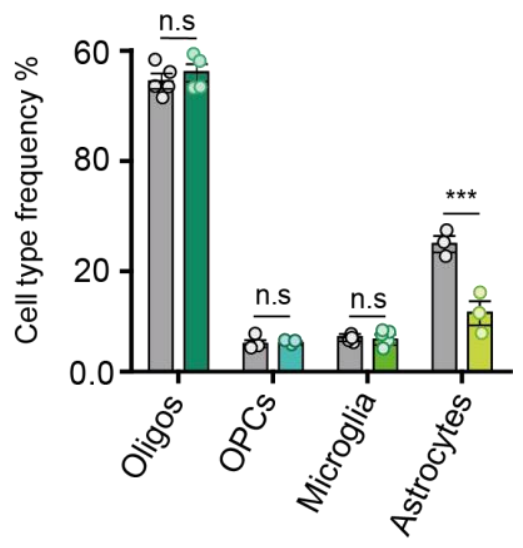

C

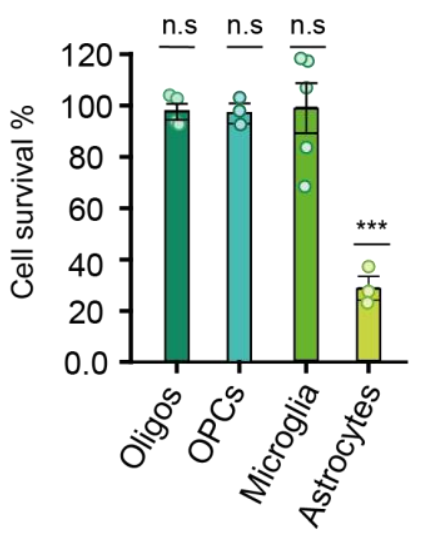

Figure 3. Effects of 24h starvation on frequency and survival of different cell types in mouse optic nerve.

(A) The images from longitudinal optic nerve sections ( 8 microm thick) of mice labled for specific cell types after $24 \mathrm{~h}$ incubation under normal (aCSF $+10 \mathrm{mM}$ glucose (Glc), left column) and starvation condition (aCSF+10 mM sucrose (Stv), right column). The blue dots showing the nucleus of the cells were stained with DAPI and the red signal is PI that has been used for detecting the dead cells during starvation. The upper row is the images from Cnp-mEos-SKL mouse line that have labeled peroxisomes specifically in their oligodendrocytes and the green signal is representing oligodendrocytes. The second row from top is related to images from mouse line which expressing YFP under control of $\mathrm{Ng} 2$ promoter $(\mathrm{Ng} 2-\mathrm{Yfp})$ and the green color is showing oligodendrocyte precursor cells (OPCs). The third row is the images from mouse line with labeled microglia and the green signal is coming from GFP protein is expressed in Microglia under control of Cxcr promoter (Cxcr-Gfp). The last row of the images is related to Aldh1L1-Gfp (ALDG) mouse line with labeled astrocytes (green signal) (Scale bar: $50 \mu m$, frame Size: 200*150 $\mathrm{mm}$ ). (B) Is showing the quantification of different cell type population (the ratio of GFP or YFP (each cell type) / the total number of cells (DAPI) in whole optic nerve section) after $24 \mathrm{~h}$ incubation under normal (GIc) or starvation condition (Stv). (C) Showing the survival of different cell types in the whole longitudinal section from the optic nerve expressed in percent. The survival rate for each cell type was calculated by dividing the number of 
live cells for each cell type after $24 \mathrm{~h}$ starvation with the frequency of each cell type after $24 \mathrm{~h}$ incubation under normal conditions. (8-9 weeks old, male or female, statistics: t-test, error bars: mean+/-SEM, n: $\geq 3$ ).

\subsubsection{Why do astrocytes die in the absence of glucose?}

In order to see why astrocytes are dying, alternative conditions including low concentration of glucose or Beta-hydroxybutyrate were applied. Two possibilities for astrocyte death were assumed: the lack of glucose that could be important for pentose phosphate pathway or the lack of available metabolites. Our data revealed both metabolites can support the survival of the nerve cells under starvation conditions.

A

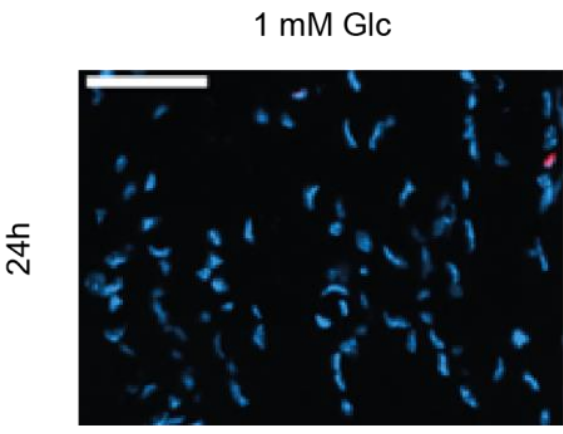

$1.5 \mathrm{mM} \mathrm{HB}$

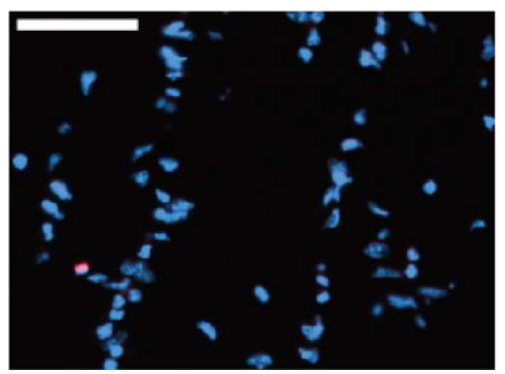

B

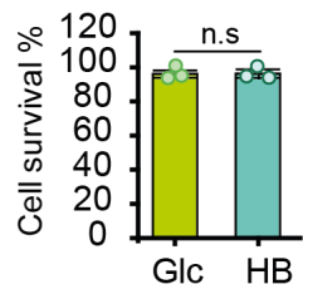

Figure 4. Presence of $1 \mathrm{mM}$ glucose or $1.5 \mathrm{mM}$ beta-hydroxybutyrate is enough for supporting the survival of optic nerve cells.

(A) The images from longitudinal optic nerve sections (8-micron thick) of wild type mice after $24 \mathrm{~h}$ incubation in the presence of aCSF $+1 \mathrm{mM}$ glucose(left) or $+1.5 \mathrm{mM}$ beta-hydroxybutyrate (right). The blue dots showing the nucleus of the cells were stained with DAPI and the red signal is PI that has been used for detecting cells death (Scale bar: $50 \mu \mathrm{m}$, frame Size: 200*150 $\mu \mathrm{m}$ ). (B) Is showing the quantified cells death in the whole longitudinal section from the optic nerve expressed in percent (ratio of PI / DAPI) after 24h incubation. (Wild type mice, 8-9 weeks old, male or female, statistics: t-test, error bars: mean+/-SEM, n:3).

\subsection{Myelin-derived fatty acuds as a source of energy under starvation conditions}

In the second section, evidence suggesting that FA metabolism and myelin degradation are involved in supporting the survival of optic nerve cells under starvation conditions will be presented. The results obtained from perturbing FA oxidation under starvation conditions by removing oxygen from the incubation solution or by applying beta-oxidation inhibitors: 


\subsubsection{Effect of beta oxidation inhibitors on survival of optic nerve cells under starvation- proteomics study}

Perturbing beta-oxidation in starved nerves followed by protein expression studies suggests FAs support cell survival under starvation condition. Our previous data showed optic nerve cells survive for a long time (16h) in the absence of glucose (Fig.3), suggesting alternative sources of energy, such as FAs, to support cell survival. Under starvation conditions, there are two possible sources of glucose for the nerve: stored glycogen in astrocytes (Wender et al., 2000) and products of gluconeogenesis. Since FA degradation depends on the presence of oxygen while glucose can produce ATP through glycolysis, to exclude the possible role of glucose in this long-term support, the nerves were incubated under hypoxia condition. Our data revealed that when glucose is available, majority of cells survive (around $85 \%$ ) under hypoxic condition, whereas hypoxia induces massive cell death in incubated nerves under starvation conditions (Fig. 5A and B). These pieces of evidence support the possible role of FA degradation in ATP production.

A

GIC

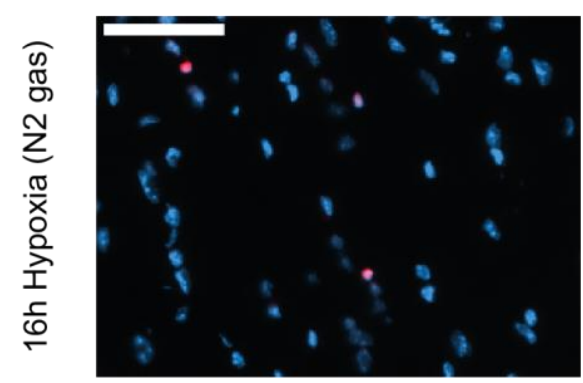

Stv

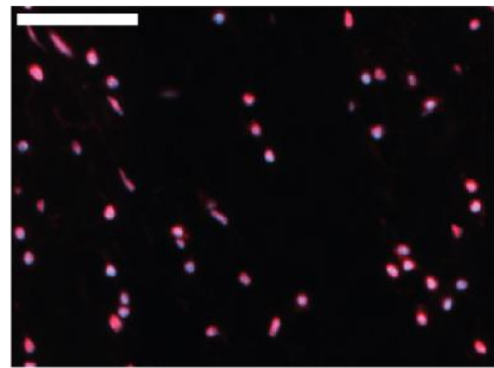

B

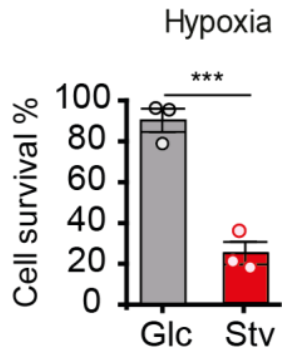

Figure 5. Hypoxia effect on survival of optic nerve cells incubated in aCSF+ $10 \mathrm{mM}$ glucose (Glc) / or $10 \mathrm{mM}$ sucrose (Stv) for $16 \mathrm{~h}$.

(A) The images from longitudinal optic nerve sections (8-micron thick) of wild type mice after $16 \mathrm{~h}$ incubation under hypoxic condition (by gassing the solution with 95\% N2 + 5\% CO2) in aCSF + $10 \mathrm{mM}$ glucose (left) or + $10 \mathrm{mM}$ sucrose (right). The blue dots showing the nucleus of the cells were stained with DAPI and the red signal is PI that has been used for detecting cells death (Scale bar: $50 \mu \mathrm{m}$, frame Size: 200*150 $\mu \mathrm{m}$ ). (B) Representing the quantified cells survival in the whole longitudinal section from the optic nerve expressed in percent (ratio of PI / DAPI) after 16h incubation. (Wild type mice, 8-9 weeks old, male or female, statistics: t-test, error bars: mean+/SEM, $\mathrm{n}: 3)$. 
Studies on isolated rat brain mitochondria had suggested 3-keto-acyl-CoA thiolase is the rate limiting enzyme for brain FA beta-oxidation (Yang et al., 1987) and 4-Bromocrotonic acid (4-Br) has been reported to be a specific inhibitor for this enzyme (Olowe and Schulz, 1982). To investigate whether FA degradation is involved in supporting the survival of nerve cells under starvation conditions, the nerves were incubated in aCSF+10 mM glucose or sucrose in the presence of $25 \mu \mathrm{M}, 4-\mathrm{Br}$ for $16 \mathrm{~h}$ and were stained with PI. Our results revealed that inhibiting mitochondrial beta-oxidation under starvation conditions induces massive cell death while the presence of glucose renders the inhibitor ineffective (Fig 6A and B). These observations clearly show that the inhibitor is not toxic to the cells and that FAs and their derivatives are catabolized in glial cells for ATP production under starvation conditions.

A

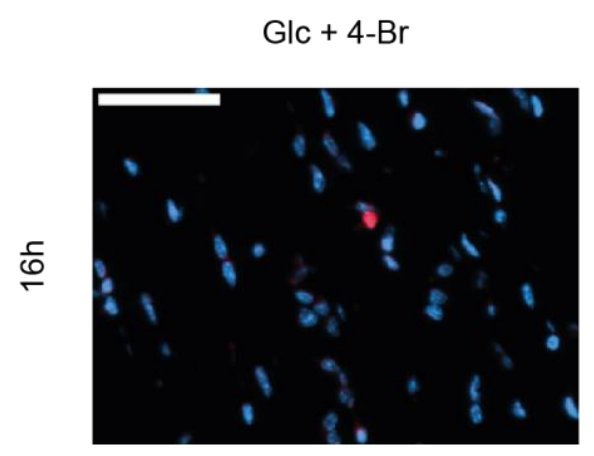

Stv $+4-B r$

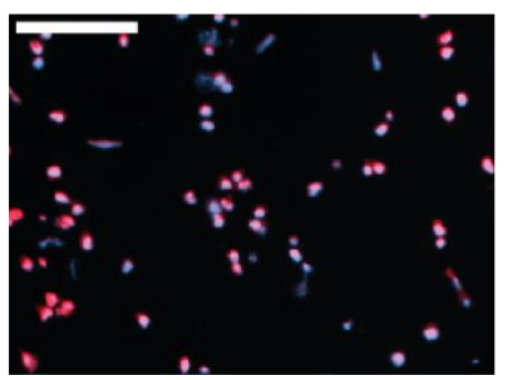

B

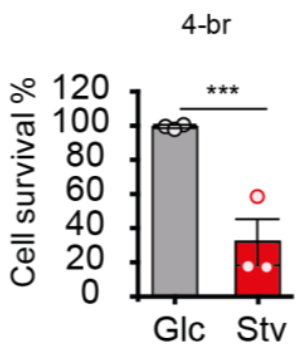

Figure 6. Effect of mitochondrial beta-oxidation inhibition on survival of optic nerve cells incubated in aCSF+10 mM glucose (GIc) / or $10 \mathrm{mM}$ sucrose (Stv) for $16 \mathrm{~h}$.

(A) The images from longitudinal optic nerve sections (8-micron thick) of wild type mice after 16h incubation in aCSF $+10 \mathrm{mM}$ glucose(left) or $+10 \mathrm{mM}$ sucrose (right) in the presence of $25 \mu \mathrm{M}$ 4-bromocrotonic acid (4-Br), specific inhibitor for mitochondrial beta-oxidation and ketolysis. The blue dots showing the nucleus of the cells were stained with DAPI and the red signal is PI that has been used for detecting cell death (Scale bar: $50 \mu \mathrm{m}$, frame Size: $200 * 150 \mu \mathrm{m}$ ). (B) Representing the quantified cell survival in the whole longitudinal section from the optic nerve expressed in percent (ratio of PI / DAPI) after 16h incubation. (Wild type mice, 8-9 weeks old, male or female, statistics: t-test, error bars: mean+/-SEM, n:3).

It is generally believed there is an interaction between peroxisomes and mitochondria, and that peroxisomes provide metabolites to mitochondria for degradation and ATP production. To see if peroxisomal beta-oxidation inhibition affects survival of optic nerve cells, the nerves were incubated in the presence of $5 \mu \mathrm{M}$ Thioridazine (Thio). Although the 
mechanism of action for thioridazine is unknown, it has been accepted as a specific inhibitor for peroxisomal beta-oxidation (Van den Branden and Roels, 1985) and there has not been any reports on its effects on mitochondrial beta-oxidation until now. The obtained data from incubated nerves in the presence of $5 \mu \mathrm{M}$ thioridazine did not affect viability of cells either under normal or under starvation conditions (fig. 7A and B). These observations suggest that inhibiting beta-oxidation in peroxisomes does not result in a drop in ATP production in mitochondria below the minimum level needed for cell survival.

A

Glc + Thio

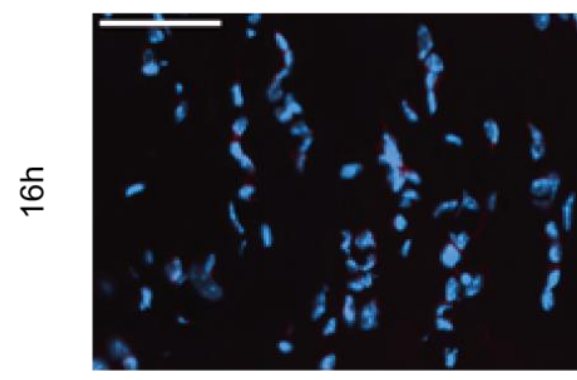

Stv + Thio

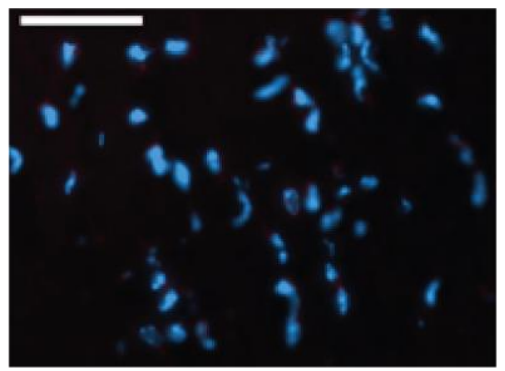

B

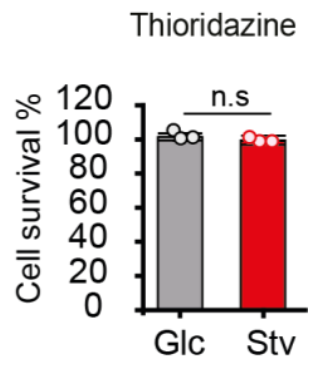

Figure 7. Effect of peroxisomal beta-oxidation inhibition on survival of optic nerve cells incubated in aCSF $+10 \mathrm{mM}$ glucose (Glc)/ or $10 \mathrm{mM}$ sucrose (Stv) for $16 \mathrm{~h}$.

(A) The images from longitudinal optic nerve sections (8-micron thick) of wild type mice after $16 \mathrm{~h}$ incubation in aCSF $+10 \mathrm{mM}$ glucose (left) or $+10 \mathrm{mM}$ sucrose (right) in the presence of $5 \mu \mathrm{M}$ Thioridazine (Thio), specific inhibitor for peroxisomal beta-oxidation. The blue dots showing the nucleus of the cells were stained with DAPI and the red signal is PI that has been used for detecting cell death (Scale bar: $50 \mu \mathrm{m}$, frame Size: 200*150 $\mu \mathrm{m}$ ). (B) Representing the quantified cell survival in the whole longitudinal section from the optic nerve expressed in percent (ratio of PI / DAPI) after 16h incubation. (Wild type mice, 8-9 weeks old, male or female, statistics: t-test, error bars: mean+/-SEM, n:3).

In order to see if there is a change in expression of proteins involved in metabolism, proteomic analysis was performed. Thanks to the MPI-EM proteomics facility, Dr. Olaf Jahn and Doerte Hesse for analyzing the samples. Autophagy is induced by glucose deprivation, therefore we expected to see regulation of gene involved in this process. Since it has been reported that ATP is needed for autophagy (Plomp et al., 1987), a low level of glucose was applied as a starvation condition in this experiment. In the previous section, the nerve incubated for $24 \mathrm{~h}$ in the presence of $1 \mathrm{mM}$ glucose did not did not show cell death (Fig.4). 
Therefore, the optic nerves from 2-month-old wild type mice were incubated for $24 \mathrm{~h}$ in the presence of $10 \mathrm{mM}$ glucose as the normal condition and $1 \mathrm{mM}$ of glucose as the starvation condition. The analyzed data revealed a change in expression of several genes involved in glucose uptake and utilization, autophagy, lipid metabolism, and FA transport. In general, these results imply that 1) glucose uptake has increased and glucose utilization has decreased, 2) autophagy has been induced, 3) and FA transport and catabolism have been upregulated (Fig.8).

A
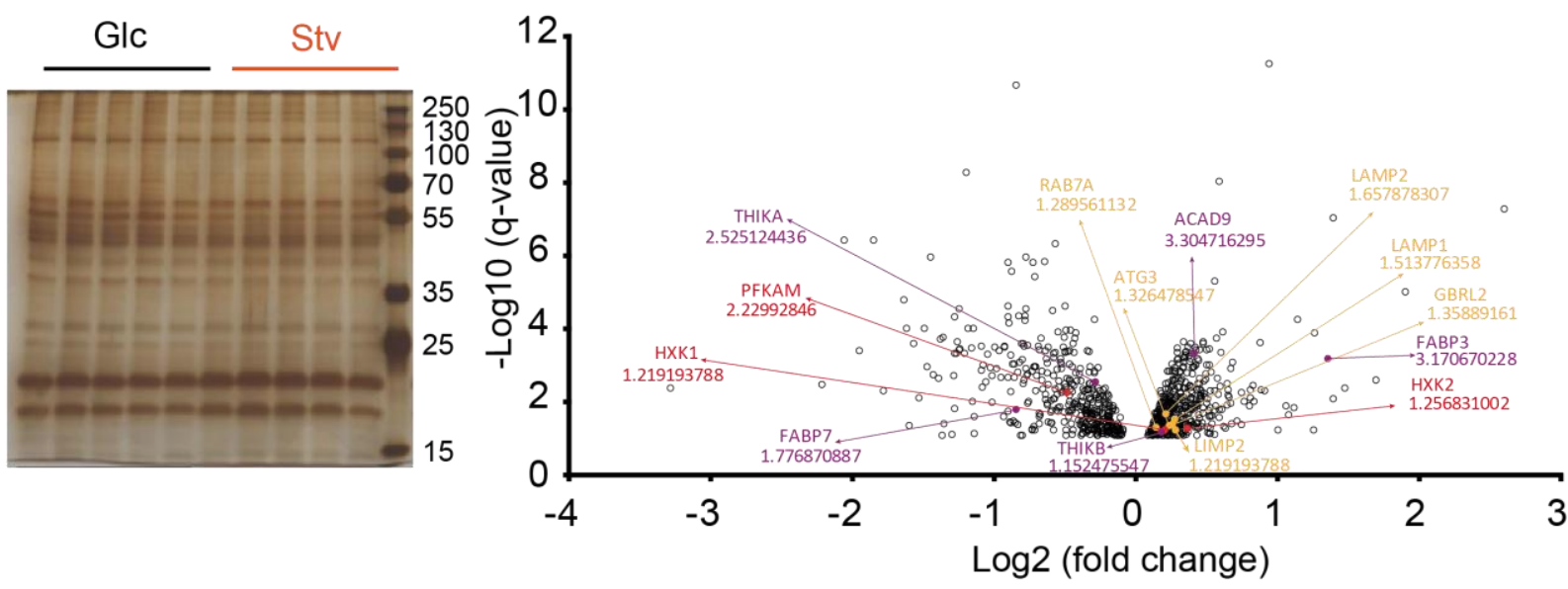

Figure 8. Effect of 16h starvation on protein expression profile of optic nerve cells (incubated in aCSF+ $10 \mathrm{mM}$ glucose (Glc)/ or $1 \mathrm{mM}$ glucose (Stv)).

(A) Silver stained gel of optic nerve lysate after $24 \mathrm{~h}$ incubation in aCSF+ $10 \mathrm{mM}$ glucose (Glc) / or $1 \mathrm{mM}$ glucose (Stv). (B) Showing the changes in protein expression of optic nerve cells after 16h incubation in aCSF $+1 \mathrm{mM}$ glucose (Starvation) in comparison with aCSF $+10 \mathrm{mM}$ glucose. The purple circles are showing proteins involved in beta-oxidation of FAs and their intracellular trafficking. The red circles are showing proteins involved in glucose utilization and the orange ones are proteins involved in autophagy. (Wild type mice, 8-9 weeks old, male or female, statistics: t-test, error bars: mean+/-SEM, n:5 biological replicate and 2 technical replicates).

\subsubsection{Effect of starvation on myelin thickness (ex vivo)}

It is known that ketone bodies produced in the liver as derivatives of FAs support brain metabolism during starvation. However, it has not yet been shown in ex vivo experiments and thus raised the question where FAs has been stored. Considering the fact that oligodendrocytes have a long survival capacity starvation conditions (Fig. 3) and that myelin is rich in FA, we hypothesized that endogenous sources of myelin FAs play a role in survival of the cells in 
mouse optic nerve under starvation condition. To verify our hypothesis, the optic nerves from wild type mice at two months of age were incubated in aCSF+ $10 \mathrm{mM}$ glucose /or sucrose for 16h and subjected to electron microscopy. Thanks to Dr. Weibke Möbius, Torben Ruhwedel and Boguslawa Sadowski in the electron microscopy (EM) facility in MPI-EM for imaging the samples. The provided images by the EM facility were used for g-ratio quantification as a readout for myelin thickness. Although the average g-ratio of starved nerves was not significantly different (data not shown), the number of axons with a thinner myelin (g-ratio between 0.8-1) has significantly increased in the absence of glucose (Fig. 9A-C). This observation verifies that myelin is degraded under starvation conditions.

A

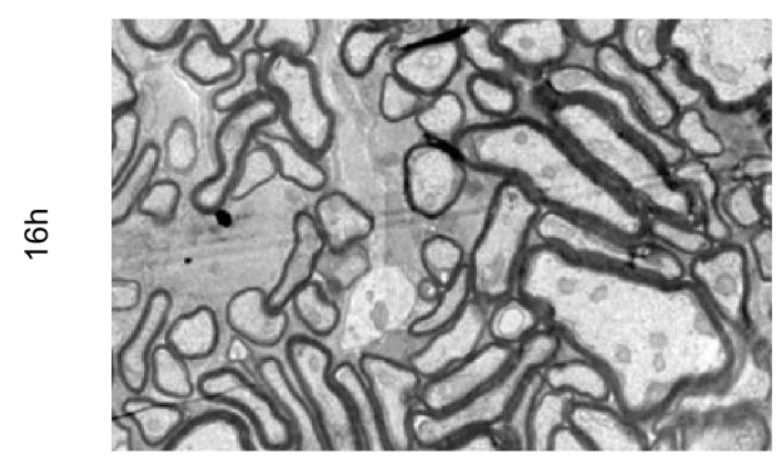

B

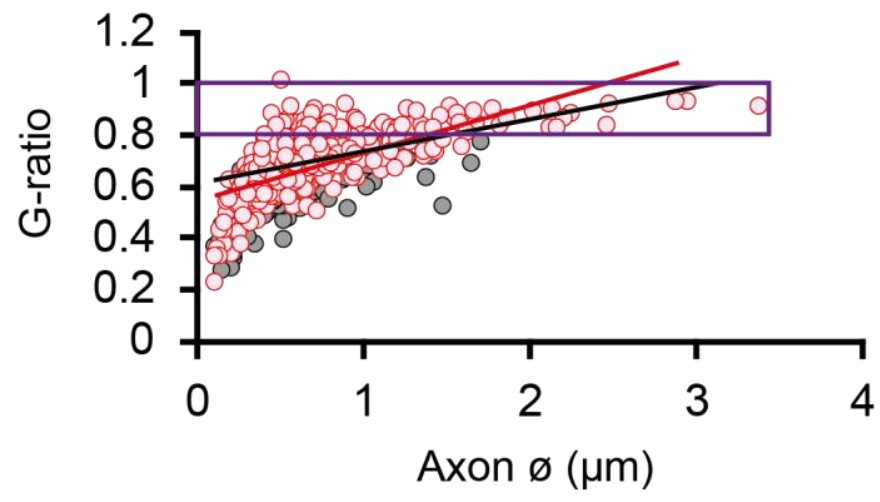

Stv

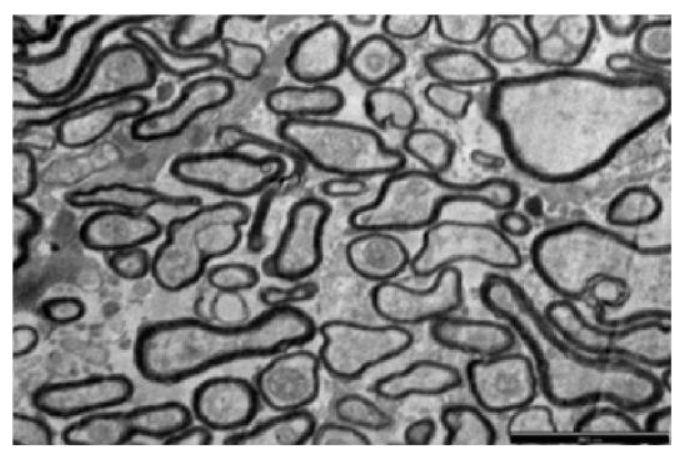

C

Figure 9. Effect of $16 \mathrm{~h}$ starvation (aCSF $+10 \mathrm{mM}$ sucrose (Stv)) on thickness of myelin in acutely isolated optic nerve.

(A) Micrographs obtained from cross sections of mouse optic nerve after $16 \mathrm{~h}$ incubation under normal (aCSF +10 mM glucose, upper image) and starvation condition (aCSF + 10 mM sucrose, lower image) (scale bar: $2000 \mathrm{~nm}$ ). (B) showing the calculated g-ratio for obtained images by dividing the diameter of outer layer of myelin to the diameter of axon+myelin. (C) Representing the difference in the number of axons with g-ratio above 0.8 between 
the nerves after $16 \mathrm{~h}$ incubation under normal (aCSF+10 mM glucose) and starvation condition (aCSF+ $10 \mathrm{mM}$ sucrose). (Wild type mice, 8-9 weeks old, male or female, statistics: t-test, error bars: mean+/-SEM, n:3).

\subsection{Role of fatty acid metabolism in supporting nerve function under starvation condition}

In this section of the results, ex vivo electrophysiology of optic nerve and monitoring of ATP levels in axons were performed to investigate the effect of FA metabolism under starvation conditions on mouse optic nerve function. In the previous two sections of the results, the role of FA metabolism on the survival of optic nerve cells was studied. As we observed in the previous section, nerve cells can stay alive for long in the absence of glucose (Fig. 2). However, the energy that is needed for the nerve to exert its function is much higher than the level needed for cells to survive. Indeed, it has been reported that the nerve function declines shortly ( $15 \mathrm{~min})$ after switching to aglycemic condition (Brown et al., 2003; Trevisiol et al., 2017). Therefore, it is necessary to find the starvation condition for recording the nerve function. The term starvation (or threshold) condition in electrophysiology experiments in this project is referred to a combination of glucose concentration and frequency of stimulation in which the optic nerve is still functioning while it is under metabolic stress.

Briefly, the optic nerve of the mouse is clamped with suction electrodes and the nerve is electrically stimulated from one end and the signal is recorded using recording electrodes at the other end. The recorded signal has three peaks related to different groups of axons with different signal propagation speeds. Since the signal results from the function of all the axons in the nerve, it is called compound action potential (CAP) and the area under the signal is referred to CAP area. By recording the nerve function during an experiment and plotting the calculated CAP area for every single trace over time it is possible to monitor the changes in nerve function during an experiment. in the next step, the calculated area could be normalized to the baseline condition, the optimal condition for nerve function. Using the optic nerve from mice that genetically encode a FRET sensor for ATP in their neurons (Trevisiol et al., 2017), we were able to monitor both function of the nerves and ATP level in axons (confocal microscopy) whenever needed. 


\subsubsection{Finding the threshold condition for optic nerve function}

A three-step protocol was designed such that the first two steps were common among all recording protocols unless otherwise stated. It has been reported the glycogen content of the optic nerve after $2 \mathrm{~h}$ incubation is equilibrated to extracellular glucose concentration (Brown et al., 2003), in order to make sure the glycogen content of the nerve is equally adjusted to the available glucose, the baseline was recorded always in aCSF $+10 \mathrm{mM}$ glucose for $2 \mathrm{~h}$ as the first step of protocol. The protocol was followed by $5 \mathrm{~min}$ aglycaemia, to partially remove the stored glycogen. Since glycogen depletion results in an insult to rat optic nerve (Wender et al., 2000), the recording was continued in aCSF $+10 \mathrm{mM}$ glucose (third step of the protocol) to see if nerve function declines because of such damage. The recorded data in the presence of $10 \mathrm{mM}$ glucose revealed 5-min glycogen depletion does not affect the nerve function adversely. It should also be noted that since the signal was stable in the presence of $10 \mathrm{mM}$ glucose, this can exclude technical influence on data obtained (Fig. 10A).

To find the threshold condition, the third step of the protocol was replaced by different concentrations of glucose (instead of $10 \mathrm{mM}$ glucose) including 2-, 2.7-, 3-, 3.3- and 3.7-mM glucose. Our data revealed the nerve function for concentrations of glucose above $2.7 \mathrm{mM}$ glucose during the $\sim 6.5 \mathrm{~h}$ recording is not significantly different from optimal condition $(10 \mathrm{mM}$ glucose). In addition, $2 \mathrm{mM}$ glucose was not sufficient for the nerve function and the CAP was declining much faster in comparison to higher glucose concentrations (Fig. 10A and B). Therefore, concentrations between $2.7 \mathrm{mM}$ and $3.3 \mathrm{mM}$ could be used as threshold concentration for glucose.

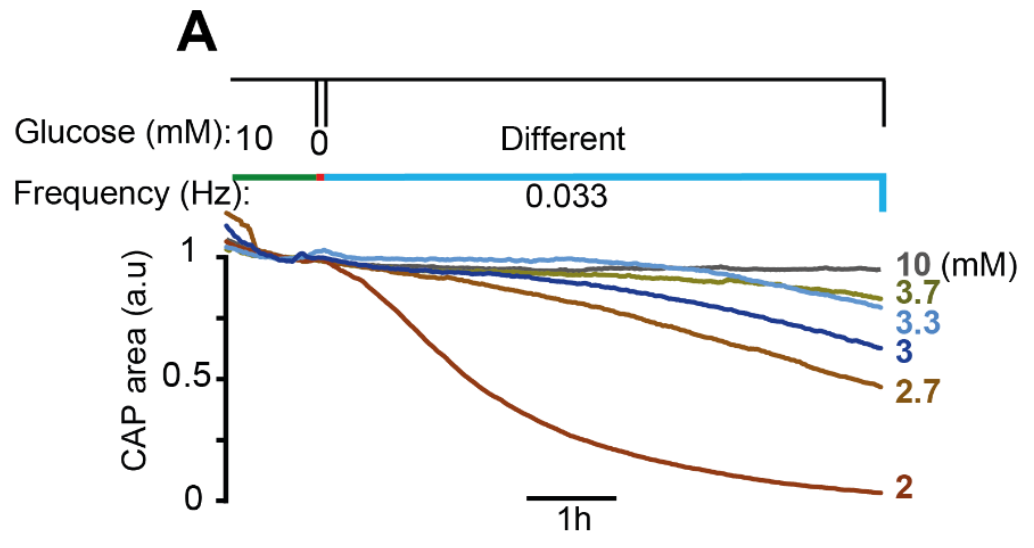


Figure 10. Optic nerve function at different concentrations of glucose obtained by ex vivo electrophysiology using suction electrodes to find the lowest glucose concentration needed nerve function.

(A) The compound action potential area (CAP area) has been calculated for every single trace during the experiment and plotted over time and for each condition average of calculated CAP area has been represented. Function of the nerve has been recorded at different concentrations of glucose including 10, 3.7, 3.3, 3, 2.7 and 2 $\mathrm{mM}$ that the related CAP area has been depicted in curves with gray, green, light blue, dark blue, light brown and dark brown colors respectively. (B) Showing the calculated area under the obtained curves in (A) for each glucose concentration (selected time window: $2 \mathrm{~h}: 5 \mathrm{~min}$ till end). (Wild type mice, 8-9 weeks old, male or female, statistics: statistical significance in comparison with $10 \mathrm{mM}$ glucose, t-test, error bars: mean+/-SEM, $\mathrm{n} \geq 3$ )

To find the threshold condition for stimulation frequency, the nerve function was recorded in aCSF+3.3 mM glucose and different frequencies were applied for nerve stimulation. The recorded nerve function stimulated with frequencies between $0.033-1 \mathrm{~Hz}$ showed when frequencies higher than $0.066 \mathrm{~Hz}$ is applied for stimulation, the CAP decline significantly faster in comparison to $0.033 \mathrm{~Hz}$ (Fig. $11 \mathrm{~A}$ and B).

Using the data obtained from mouse optic nerve recordings at different glucose concentrations and different frequencies (Fig. 10 and Fig. 11), a protocol was designed to investigate the role of FA metabolism in nerve function under starvation conditions by applying specific inhibitors. This protocol also includes three steps, the first two steps were $2 \mathrm{~h}$ baseline recording $(\mathrm{aCSF}+10$ $\mathrm{mM}$ glucose, $0.033 \mathrm{~Hz}$ ), and $5 \mathrm{~min}$ aglyceamia (aCSF+ $0 \mathrm{mM}$ glucose, $0.033 \mathrm{~Hz}$ ) respectively. The third step was recording in low concentrations of glucose (2.7 $\mathrm{mM}+/$ - inhibitor) and a RAMP protocol composed of $0.2 \mathrm{~Hz}$ (for $2.5 \mathrm{~h}), 1 \mathrm{~Hz}(54 \mathrm{~min}), 3 \mathrm{~Hz}$ (30 min) and $7 \mathrm{~Hz}$ (30 min) for stimulating the nerves.

\section{A}

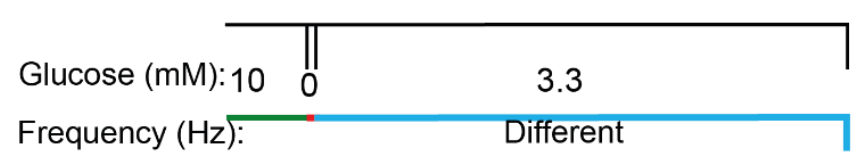

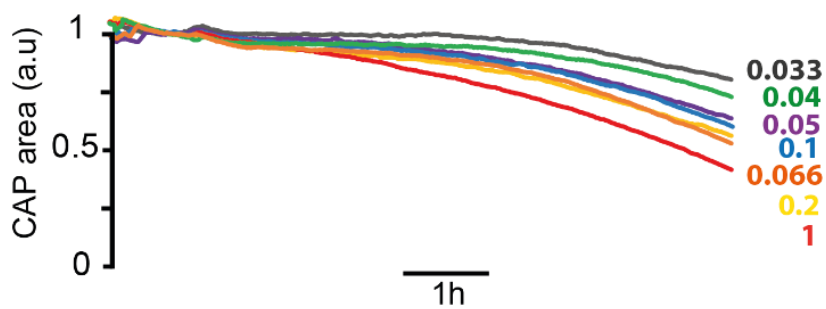

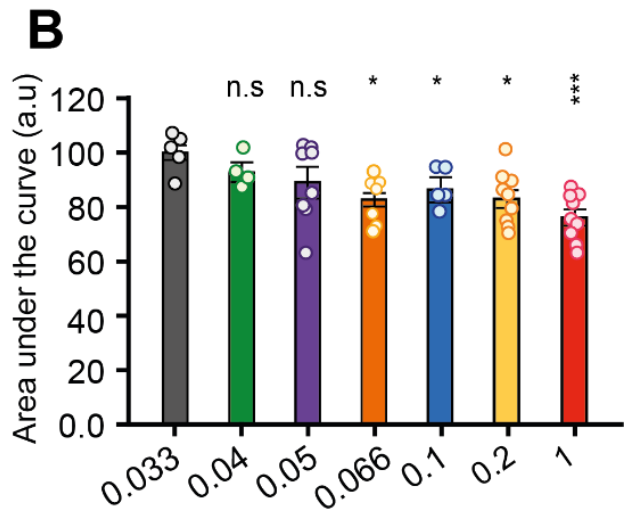

Frequency $(\mathrm{Hz})$

Figure 11. Optic nerve function at different frequencies $(\mathrm{Hz})$ obtained by ex vivo electrophysiology using suction electrodes. 
(A) The compound action potential area (CAP area) has been calculated for every single trace during the experiment and plotted over time and for each condition average of calculated CAP area has been represented. Function of the nerve has been recorded in aCSF+ 3.3 mM glucose at different frequencies including 0.033-, 0.04, 0.05-, 0.066-, 0.1, 0.2 and $1 \mathrm{~Hz}$ that the related CAP area has been depicted in curves gray, green, purple, orange, blue, yellow and red colors respectively. (B) Showing the calculated area under the obtained curves in (A) for each frequency (selected time window: 2h:5min till end,). (Wild type mice, 8-9 weeks old, male or female, statistics: ttest, statistical significance in comparison with $0.033 \mathrm{~Hz}$, error bars: mean+/-SEM, $\mathrm{n} \geq 5$ )

\subsubsection{Beta-hydroxybutyrate supports nerve function under starvation condition}

It is well known that ketone bodies can be metabolized by brain cells during starvation. However, electrophysiological recordings have failed to show that ketone bodies are utilized for supporting nerve function (Brown et al., 2001). To see if ketone bodies are being used by brain, we applied beta-hydroxybutyrate in combination with $3.3 \mathrm{mM}$ glucose. Our data revealed beta-hydroxybutyrate $(10 \mathrm{mM})$ alone is not able to maintain nerve function; however, in combination with glucose ( $3.3 \mathrm{mM}$ glucose $+6.7 \mathrm{mM}$ beta-hydroxybutyrate) it improves the nerve function significantly (Fig. 12A and B).

\section{A}

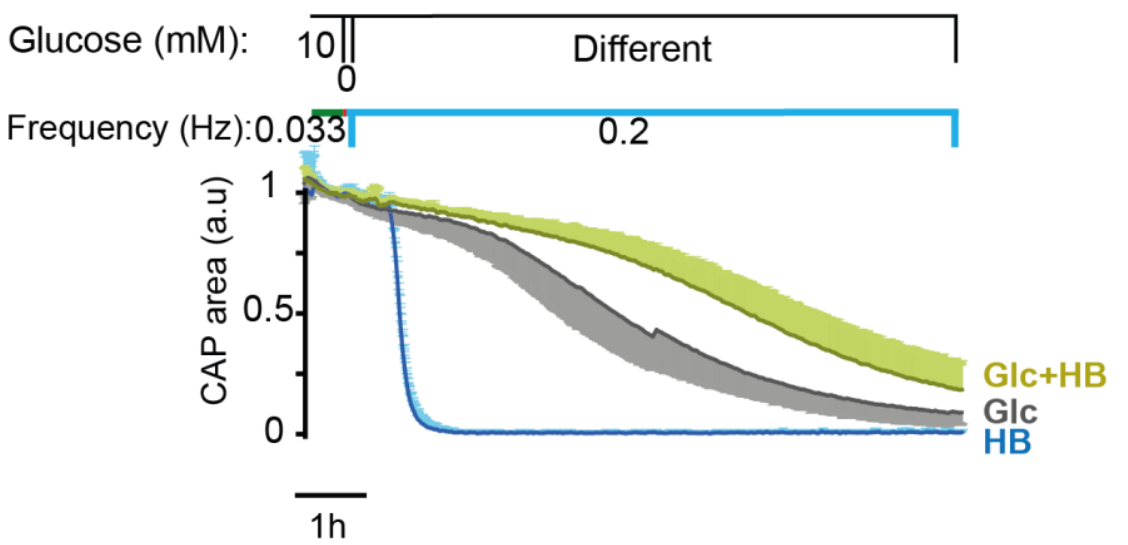

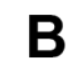

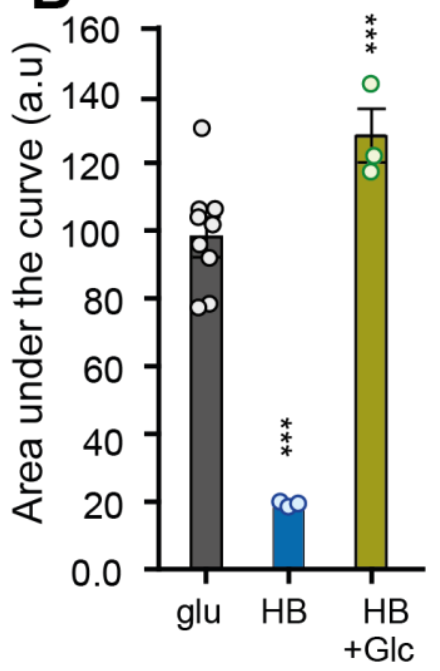

Figure 12. Support of optic nerve function by beta-hydroxybutyrate (HB) alone and in combination with glucose.

(A) Representing the optic nerve function (compound action potential (CAP area)) of wild type mice recorded after baseline (2h, $10 \mathrm{mM}$ glucose, $0.033 \mathrm{~Hz}$ ) and partial glycogen depletion ( $5 \mathrm{~min}, 0 \mathrm{mM}$ glucose, $0.033 \mathrm{~Hz}$ ) at low concentration of glucose (gray curve: $3.3 \mathrm{mM}$, Frequency of stimulation $0.2 \mathrm{~Hz}$ ), in the presence of betahydroxybutyrate (blue curve: $10 \mathrm{mM}$, Frequency of stimulation $0.2 \mathrm{~Hz}$ ) and in the presence of betahydroxybutyrate in combination with glucose (in green: $3.3 \mathrm{mM}$ glucose $+6.7 \mathrm{mM}$ beta-hydroxybutyrate (HB), Frequency of stimulation $0.2 \mathrm{~Hz}$ ). (B) Showing the calculated area under the obtained curves (selected time 
window: 2h:5min till end) in (A) for each condition. (Wild type mice, 8-9 weeks old, male or female, statistics: data were normalized to the average of $3.3 \mathrm{mM}$ glucose), t-test, error bars: mean+/-SEM, $n \geq 3$ ).

\subsubsection{Effect of blocking mitochondrial beta-oxidation on nerve function under starvation conditions}

Recording the optic nerve function under starvation conditions revealed that the CAP area declines faster when mitochondrial beta-oxidation is inhibited by applying $25 \mu \mathrm{M} 4-\mathrm{Br}$ (Fig. $13 \mathrm{~A}$ and B). At the same time by monitoring ATP level in axons, a direct correlation between ATP level and CAP decline was observed (Fig. 13C and D). These data for the first time clearly show that beta-oxidation of endogenous FA sources in the nerve are involved in supporting nerve function when glucose is not sufficient to meet energy demands. In order to normalize the ATP level in the axons, the RAMP protocol was followed by applying Azide in the absence of glucose (to block mitochondrial beta oxidation and deplete ATP in axons). In addition, a decreased recovery was observed in treated nerves with 4-Br in comparison with control after reperfusion with aCSF+10 mM glucose (Fig. 13E and F). To exclude the role of inhibitor toxicity in the observed decline, the nerve function was recorded in aCSF $+10 \mathrm{mM}$ glucose in the presence of $25 \mu \mathrm{M} 4-\mathrm{Br}$. Since the CAP is not affected by the inhibitor when the glucose level is high (10 mM) (Fig. 13G and H), it was concluded that CAP decline under starvation is not due to the toxicity of the inhibitor. 
A

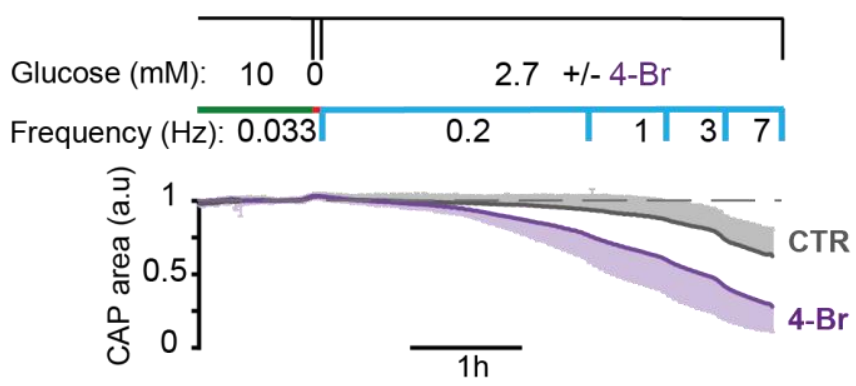

C

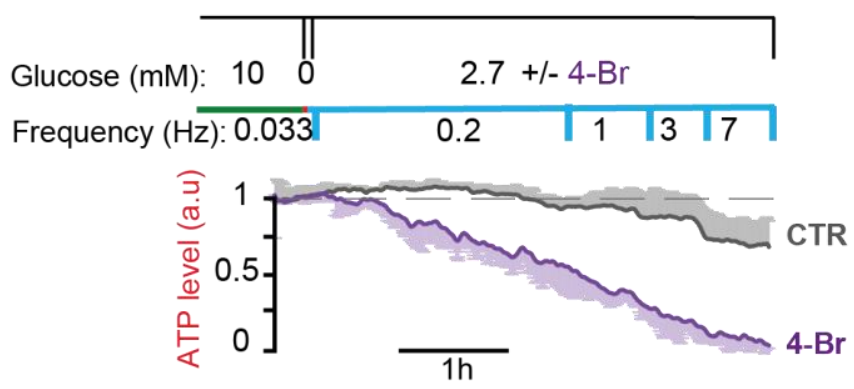

E

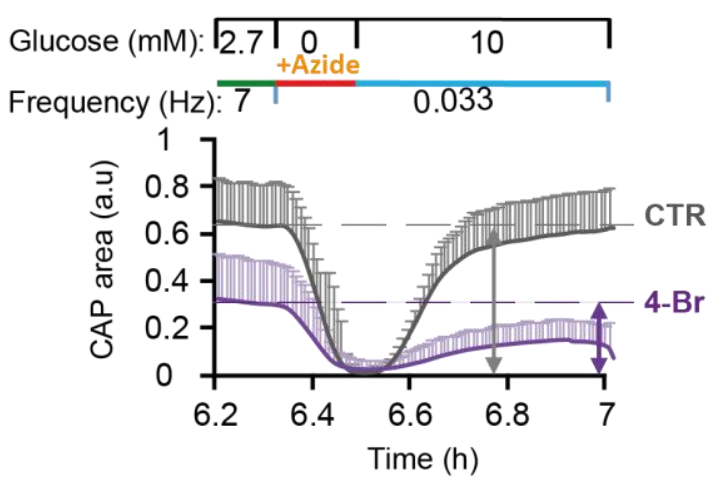

\section{G}

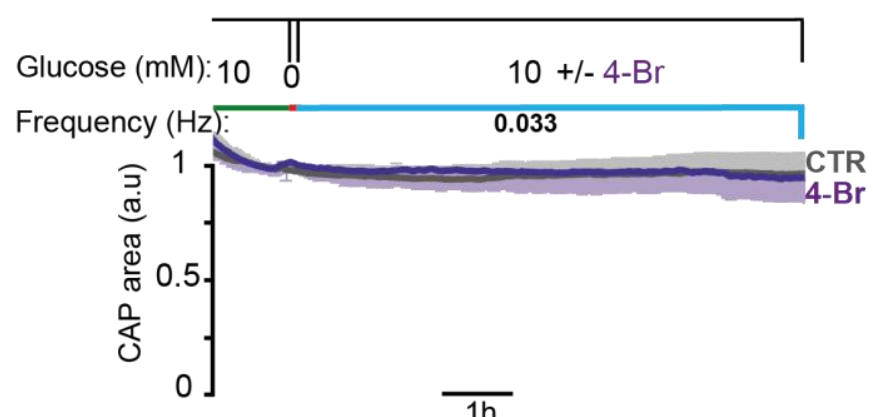

B

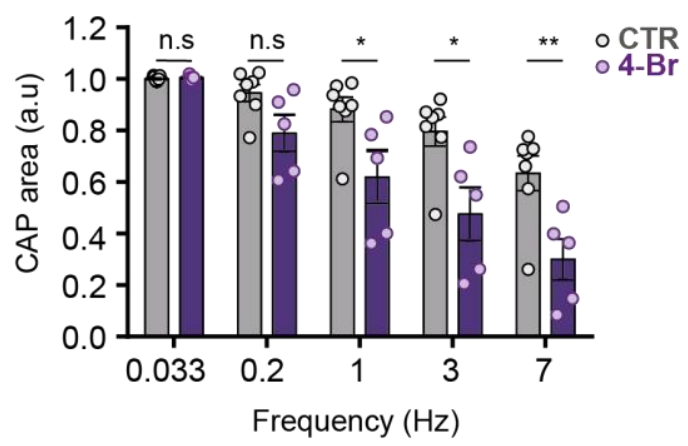

D

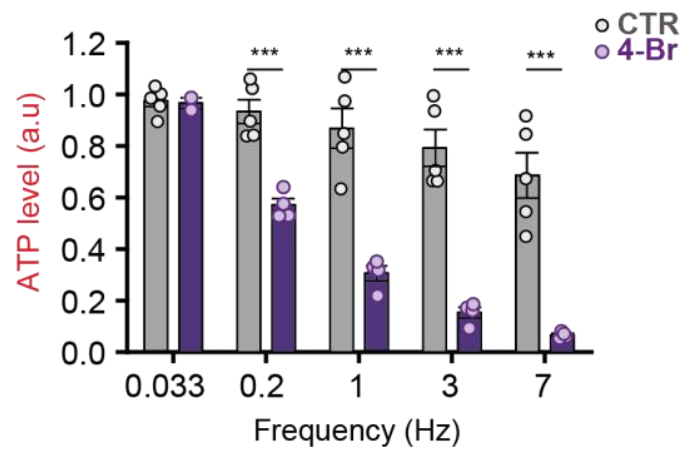

$\mathbf{F}$

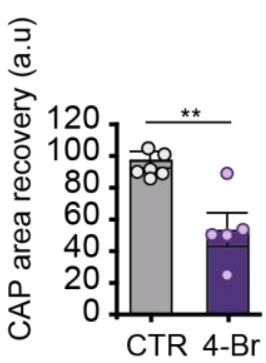

H

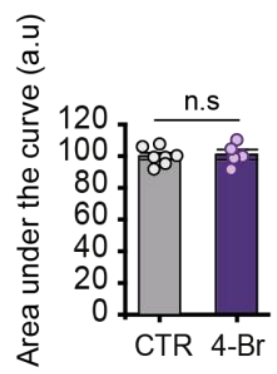

Figure 13. Effect of mitochondrial beta-oxidation inhibitor ( $25 \mu \mathrm{M} 4-\mathrm{Br})$ on optic nerve function under starvation obtained by ex vivo electrophysiology using suction electrodes.

(A) Representing the optic nerve function (compound action potential (CAP area)) of THYATPA mouse line recorded after baseline $(2 \mathrm{~h}, 10 \mathrm{mM}$ glucose, $0.033 \mathrm{~Hz}$ ) and partial glycogen depletion ( $5 \mathrm{~min}, 0 \mathrm{mM}$ glucose, 0.033 
$\mathrm{Hz}$ ) at low concentration of glucose (2.7 mM, RAMP stimulation: 0.2, 1, 3 and $7 \mathrm{~Hz}$ ) in the absence (gray curve) and presence (purple curve) of $25 \mu \mathrm{M}$ mitochondrial beta-oxidation inhibitor, 4-Bromocrotonic acid $(4-\mathrm{Br})(\mathrm{n} \geq 5)$. (B) Showing the average of CAP area for the traces have been recorded during the last 5 min of each step of RAMP protocol (0.033 (baseline), 0.2, 1, 3 and $7 \mathrm{~Hz}$ ). (C) Showing the changes in ATP level in axons of the same optic nerves that their CAP area has been shown in (A). The changes in ATP level was monitored using a genetically encoded FRET sensor in neurons (Protocol: solutions and frequency of stimulation was the same as $(A)$ ) in the presence (purple curve) and absence (gray) of 4-Bromocrotonic acid (4-Br). The curve is showing the FRET signal normalized to CFP and plotted over time ( $n \geq 4)$. (D) Showing the quantifications of the ATP level during the last 5 min of each step of RAMP protocol (FRET/CFP). (E) Representing the effect of $5 \mathrm{mM}$ Azide (aCSF+10 mM sucrose) on nerve function and nerve function recovery after reperfusion with aCSF+ $10 \mathrm{mM}$ glucose $(n \geq 5)$. ( $F)$ Showing the quantification related to nerve recovery in section (E) (CAP area after recovery / mean of CAP area obtained during the last 5 min of RAMP protocol). (G) Showing the calculated CAP area recorded from optic nerve of wild type mice in aCSF $+10 \mathrm{mM}$ glucose in the presence (red curve) and absence (gray curve) of $25 \mu \mathrm{M} 4$-Bromocrotonic acid (4-Br). The applied frequency for stimulating the nerve was $0.033 \mathrm{~Hz}(n \geq 5)$. $(\mathrm{H})$ representing the calculated area under the obtained curves (selected time window: $2 \mathrm{~h}$ : $5 \mathrm{~min}$ till end) in (G). (8-9 weeks old mice, male or female, statistics: t-test, error bars: mean+/-SEM, $n \geq 5$ ).

To see if beta-oxidation of long chain FAs in the mitochondria is involved in supporting nerve function under starvation conditions, nerve function was recorded in the presence of specific inhibitor for degradation of LCFAs, Etomoxir (Etox). Our data revealed that the CAP declines faster under starvation condition when $5 \mu \mathrm{M}$ Etox is applied (Fig.14A and B). Combining electrophysiology with ATP level monitoring in axons, our data revealed a faster decline in the CAP that is accompanied with a faster decline in axonal ATP (Fig. 14C and D). These observations clearly show that degradation of LCFAs in nerve cells is involved in supporting the nerve function under starvation. In order to normalize the ATP level in the axons, the RAMP recording was followed by ATP depletion using Azide in the absence of glucose. After nerve reperfusion with aCSF+10 mM glucose, we did not observe significant differences in nerve function recovery of Etox-treated samples compared to the control (Fig. 13E and F). To rule out the observed decline is caused by toxicity of the inhibitor, the nerve function was recorded under optimal condition (aCSF+10 mM glucose) in the presence of inhibitor, Etox. The CAP area did not show any decline in the presence of inhibitor and $10 \mathrm{mM}$ glucose (Fig. $14 \mathrm{G}$ and $\mathrm{H}$ ), speaking of the fact the inhibitor is not toxic to nerve cells. 
A

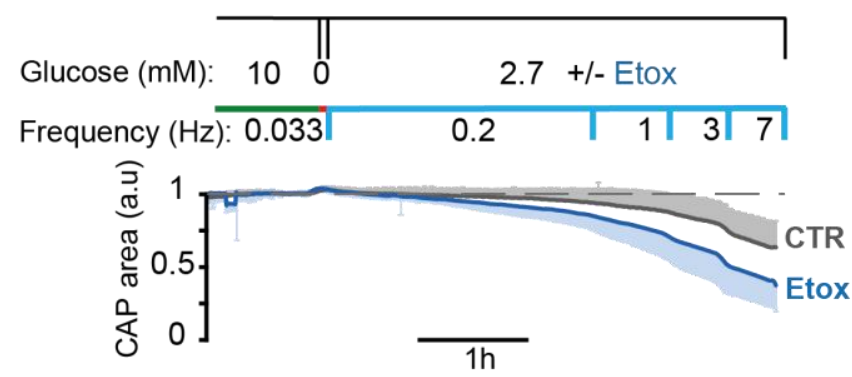

C

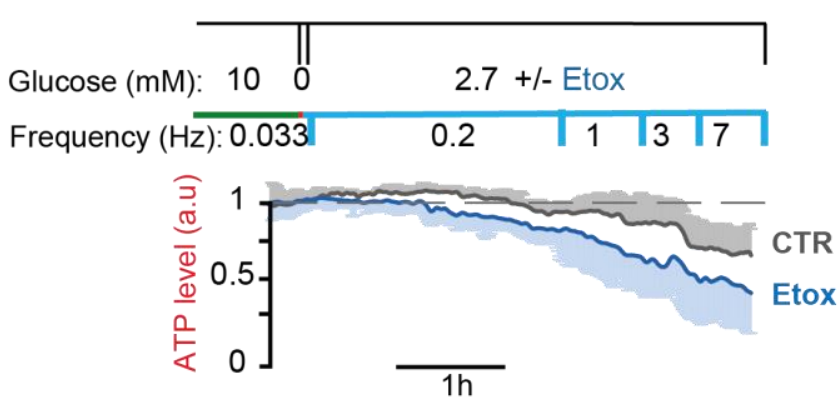

E

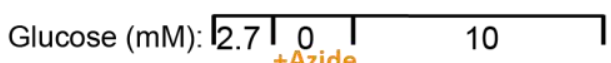

Frequency $(\mathrm{Hz}): 7 T^{\text {+Azide }}$

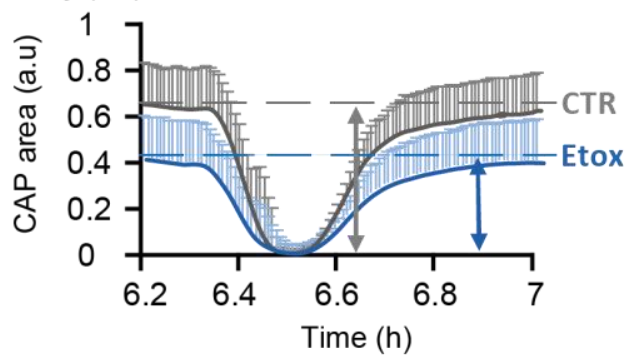

G

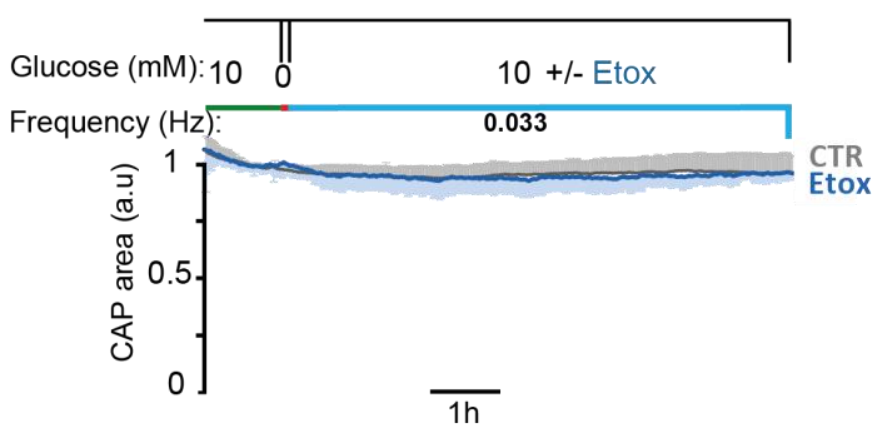

B

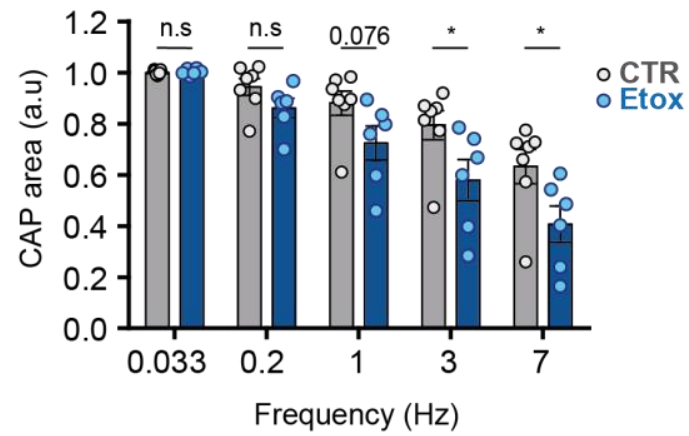

D

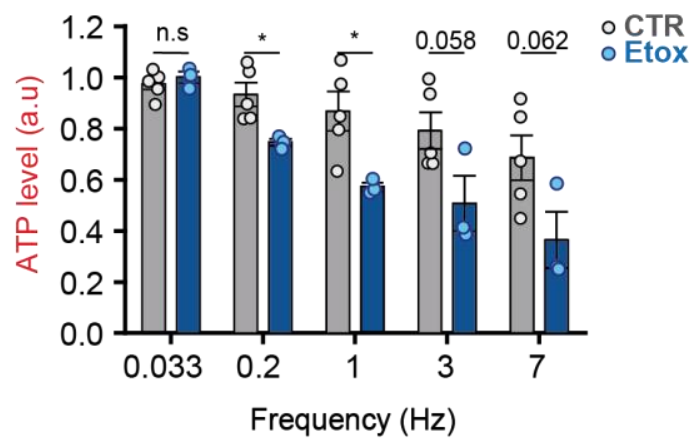

$\mathbf{F}$

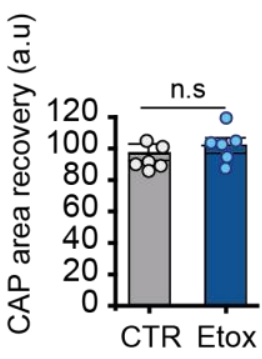

H

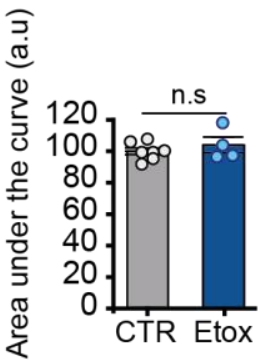

Figure 14. Effect of long chain FA beta-oxidation inhibition ( $5 \mu \mathrm{M}$ Etomoxir) on optic nerve function under starvation obtained by ex vivo electrophysiology using suction electrodes.

(A) Representing the optic nerve function (compound action potential (CAP area)) of THYATPA mouse line recorded after baseline $(2 \mathrm{~h}, 10 \mathrm{mM}$ glucose, $0.033 \mathrm{~Hz}$ ) and partial glycogen depletion ( $5 \mathrm{~min}, 0 \mathrm{mM}$ glucose, $0.033 \mathrm{~Hz}$ ) at 
low concentration of glucose (2.7 mM, RAMP stimulation: $0.2,1,3$ and $7 \mathrm{~Hz}$ ) in the absence (gray curve) and presence (blue curve) of $5 \mu \mathrm{M}$ of long chain FA beta-oxidation inhibitor, Etomoxir (Etox)( $n \geq 6)$. (B) Showing the average of CAP area for the traces have been recorded during the last $5 \mathrm{~min}$ of each step of RAMP protocol (0.033 (baseline), 0.2, 1, 3 and $7 \mathrm{~Hz}$ ). (C) Representing the changes in ATP level in axons of the same optic nerves that their CAP area has been shown in (A). The changes in ATP level was monitored using a genetically encoded FRET sensor in neurons (Protocol: solutions and frequency of stimulation was the same as $(A)$ ) in the presence (blue curve) and absence (gray) of Etomoxir (Etox). The curve is showing the FRET signal normalized to CFP and plotted over time $(n \geq 3)$. (D) Showing the quantified ATP level during the last 5 min of each step of RAMP protocol (FRET/CFP). (E) Representing the effect of $5 \mathrm{mM}$ Azide (aCSF+10 mM sucrose) on nerve function and nerve function recovery after reperfusion with aCSF+ $10 \mathrm{mM}$ glucose (n:6). (F) Showing the quantifications related to nerve recovery after Azide treatment (CAP area after recovery was normalized to the mean of CAP area obtained during the last 5 min of RAMP protocol). (G) Showing the calculated CAP area recorded from optic nerve of wild type mice in aCSF $+10 \mathrm{mM}$ glucose in the presence (red curve) and absence (gray curve) of $5 \mu \mathrm{M}$ Etomoxir (Etox). The applied frequency for stimulating the nerve was $0.033 \mathrm{~Hz}(\mathrm{n} \geq 4)$. $(\mathrm{H})$ representing the calculated area under the obtained curves (selected time window: $2 \mathrm{~h}$ : $5 \mathrm{~min}$ till end) in (G). (8-9 weeks old mice, male or female, statistics: t-test, error bars: mean+/-SEM).

\subsubsection{Effect of blocking peroxisomal beta-oxidation on nerve function under starvation condition (Thioridazine and Mfp2 KO mice)}

Peroxisomal beta-oxidation provides some metabolites to mitochondria for degradation. To see if peroxisomes are involved in supporting nerve function under starvation condition, the CAP (8-9 weeks old wild type mice) was recorded under starvation condition in the presence of $5 \mu \mathrm{M}$ Thioridazine (Thio), the specific inhibitor for peroxisomal beta-oxidation. Peroxisomal beta-oxidation inhibition resulted in a faster decrease in nerve function (Fig. 15A and B) compared to the control and a similar decline was observed in axonal ATP of the nerve (Fig. $15 C$ and $\mathrm{D})$. These data for the first time show the importance of peroxisomal beta-oxidation in optic nerve cells for nerve function under starvation. The recovery of the nerves after Azide treatment was not significantly different with respect to the control (Fig. 15E and F). The recorded CAP under optimal condition (10 mM glucose) and in the presence of inhibitor does not show a decline in nerve function (Fig. 15G and H), implying that the inhibitor is not toxic to the nerve cells. 
A

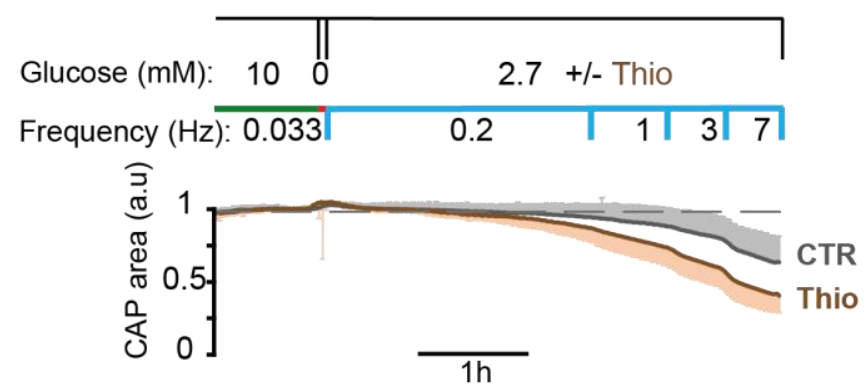

C

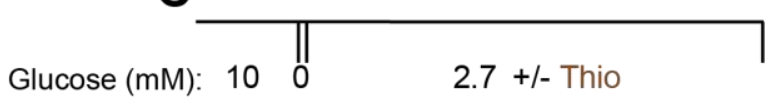

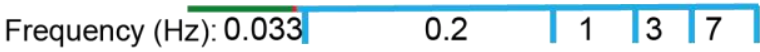

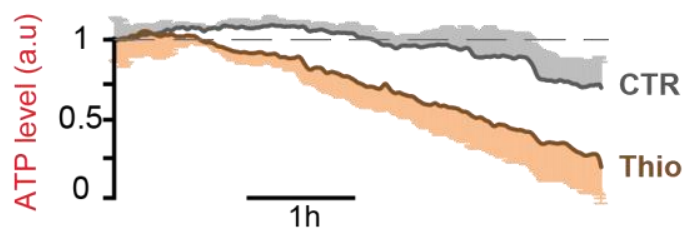

E

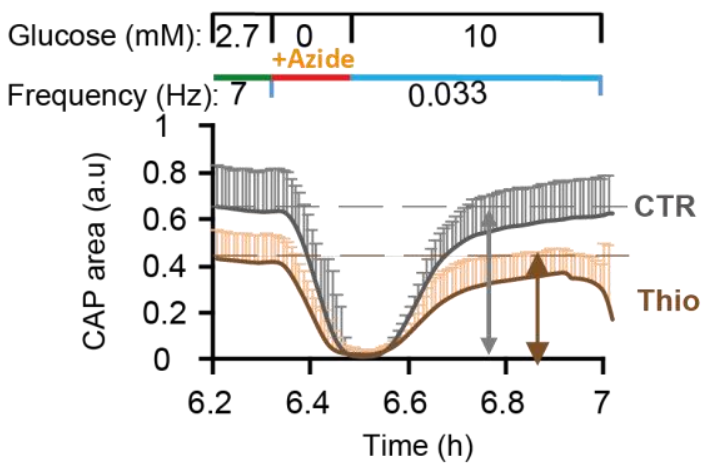

G

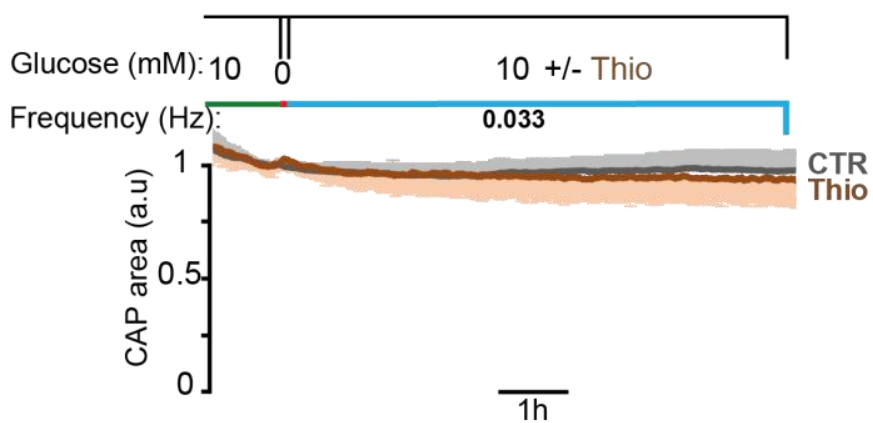

B

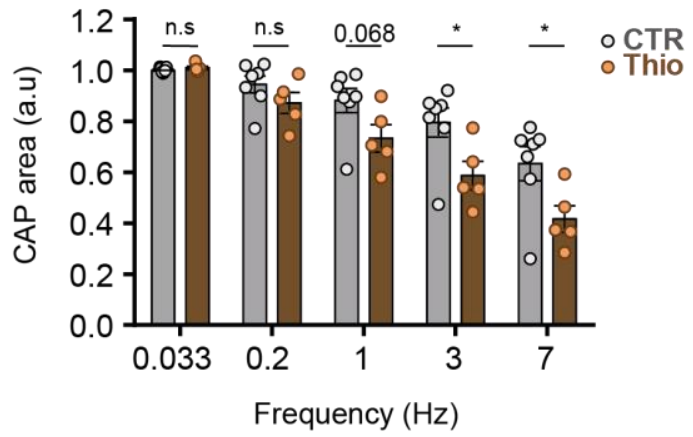

D

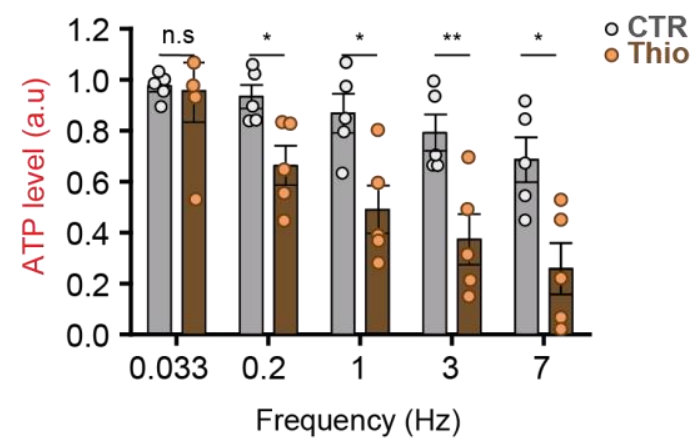

$\mathbf{F}$

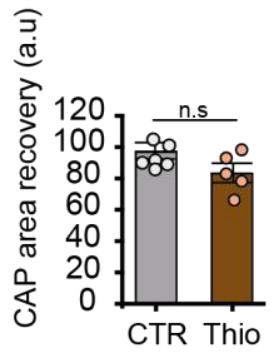

H

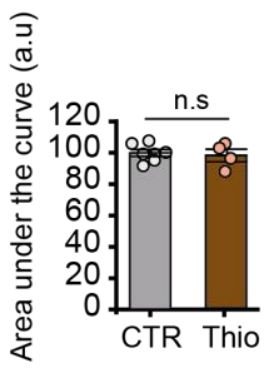

Figure 15. Effect of peroxisomal beta-oxidation inhibition using ( $5 \mu \mathrm{M}$ Thioridazine) on optic nerve function under starvation obtained by ex vivo electrophysiology using suction electrodes.

(A) Representing the optic nerve function (compound action potential (CAP area)) of THYATPA mouse line recorded after baseline $\left(2 \mathrm{~h}, 10 \mathrm{mM}\right.$ glucose, $0.033 \mathrm{~Hz}$ ) and partial glycogen depletion $\left(5 \mathrm{~min}, 0 \mathrm{mM}\right.$ glucose, $30^{-1}$ $\mathrm{Hz}$ ) at low concentration of glucose (2.7 mM, RAMP stimulation: $0.2,1,3$ and $7 \mathrm{~Hz}$ ) in the absence (gray curve) 
and presence (brown curve) of $5 \mu \mathrm{M}$ of long chain FA beta-oxidation inhibitor, Thioridazine $($ Thio) $(n \geq 5)$. (B) Showing the average of CAP area for the traces have been recorded during the last 5 min of each step of RAMP protocol (0.033 (baseline), 0.2, 1, 3 and $7 \mathrm{~Hz}$ ). (C) Representing the changes in ATP level in axons of the same optic nerves that their CAP area has been shown in (A). The changes in ATP level was monitored using a genetically encoded FRET sensor in neurons (Protocol: solutions and frequency of stimulation was the same as (A)) in the presence (brown curve) and absence (gray) of Thioridazine (Thio). The curve is showing the FRET signal normalized to CFP and plotted over time ( $n \geq 5)$. (D) Showing the quantified ATP level during the last 5 min of each frequency of RAMP protocol (FRET/CFP). (E) Representing the effect of $5 \mathrm{mM}$ Azide (aCSF+10 mM sucrose) on nerve function and nerve function recovery after reperfusion with aCSF+ $10 \mathrm{mM}$ glucose $(n \geq 5)$. (F) Quantifications related to nerve recovery after Azide treatment (CAP area after recovery normalized to the mean of CAP area obtained during the last 5 min of RAMP protocol). (G) Showing the calculated CAP area recorded from optic nerve of wild type mice in aCSF $+10 \mathrm{mM}$ glucose in the presence (red curve) and absence (gray curve) of $5 \mu \mathrm{M}$ Thioridazine (Thio). The applied frequency for stimulating the nerve was $0.033 \mathrm{~Hz}(\mathrm{n} \geq 4)$. (H) Representing the calculated area under the obtained curves (selected time window: $2 \mathrm{~h}$ : $5 \mathrm{~min}$ till end) in (G). (8-9 weeks old mice, male or female, statistics: ttest, error bars: mean+/-SEM).

By applying thioridazine, peroxisomal beta-oxidation is inhibited in all cells of the nerve universally. To investigate the role of oligodendrocyte peroxisomes in supporting nerve function under starvation condition, the optic nerve from KO mice $\left(\mathrm{MFP}^{\mathrm{fl} / \mathrm{fl}}\right.$ * $\left.\mathrm{Cnp}-\mathrm{Cre}^{+\wedge}\right)$ with perturbed peroxisomal beta-oxidation were subjected to ex vivo electrophysiology. Our data revealed, the nerve function decreases faster in MFP2 KO mice compared to the control under starvation conditions (Fig. 16A and B). These data for the first time show the importance of peroxisomal beta-oxidation products in supporting nerve function.

A

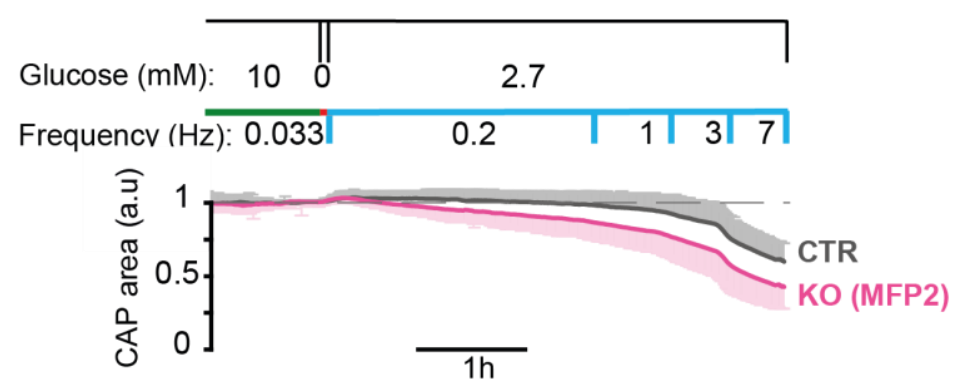

B

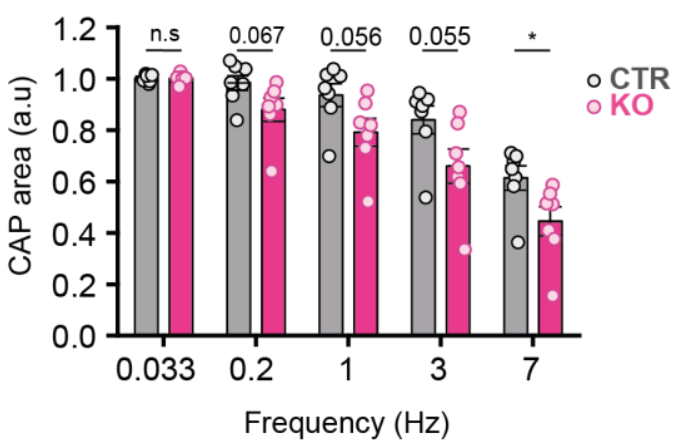

Figure 16. Effect of perturbing peroxisomal beta-oxidation in oligodendrocytes $\left(M f p 2^{f / f f} * \mathrm{Cnp}-\mathrm{Cr} e^{+/-}\right)$on optic nerve function under starvation obtained by ex vivo electrophysiology using suction electrodes.

(A) Representing the optic nerve function (compound action potential (CAP area)) of Mfp2 KO mouse line (knockout (KO) and control (CTR) has been represented in pink and gray curves respectively) recorded after baseline $(2 \mathrm{~h}, 10 \mathrm{mM}$ glucose, $0.033 \mathrm{~Hz}$ ) and partial glycogen depletion $(5 \mathrm{~min}, 0 \mathrm{mM}$ glucose, $0.033 \mathrm{~Hz}$ ) at low concentration of glucose $(3.3 \mathrm{mM}$, Stimulation frequency: $0.2 \mathrm{~Hz}$ ). (B) Showing the mean of CAP area recorded the last 5 min of each steps of RAMP protocol. (8-9 weeks old mice, male or female, statistics: t-test, error bars: mean+/-SEM, $n: 7)$. 


\subsection{Mechanism of myelin degradation (autophagy perturbation: Tfeb KO mice, Lys05) and transport of fatty acids (Octn3 KO mice)}

Using pharmacology and transgenic mice we investigated the effect of perturbing autophagy and carnitine transport in peroxisomes (OCTN3) on optic nerve function. Our data revealed that the ablation of TFEB as a master regulator of autophagy in oligodendrocytes does not affect the nerve function under starvation conditions at frequencies below $3 \mathrm{~Hz}$. When a $7 \mathrm{~Hz}$ frequency was applied in the presence of $2.7 \mathrm{mM}$ glucose the CAP slightly declined faster in Tfeb KO mice in comparison with controls, however, it was not statistically significant (Fig17. $A$ and $B)$. To see if other signaling pathways are involved in autophagy regulation in the absence of TFEB, $5 \mu \mathrm{M}$ of autophagy inhibitor was applied during the nerve recording of wildtype mice under starvations. Our data revealed a significant improvement in treated nerves at frequencies lower than $1 \mathrm{~Hz}$, while this significant improvement disappears when 3 and $7 \mathrm{~Hz}$ were applied for stimulation the nerve (Fig. 17A and B). Lys05 in nerve cells by applying Lys05 improved the nerve function when 0.2 and $1 \mathrm{~Hz}$ were applied however at higher frequencies.

Since it has been reported that OCTN3 is involved in peroxisomal FA metabolism the optic nerve from the Octn $3^{f / f t}$ * Cnp-Cre mouse line was subjected to ex-vivo electrophysiology. Our data revealed that the inactivation of OCTN3 in oligodendrocytes does not affect the nerve function in the presence of $3.3 \mathrm{mM}$ glucose (Fig. 17E and F). 


\section{A}

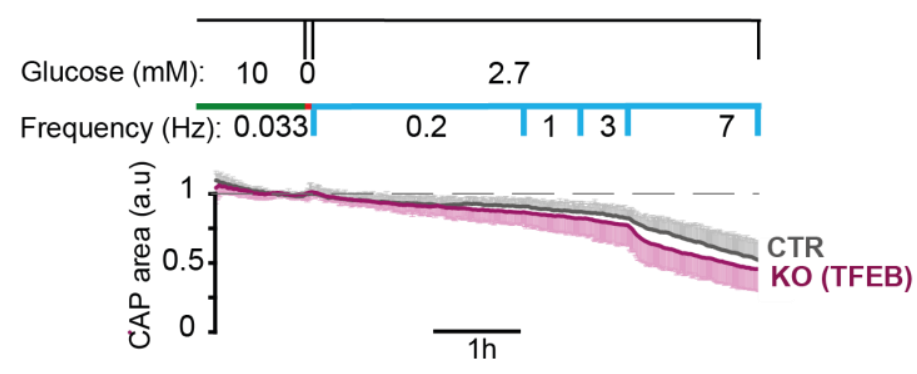

C

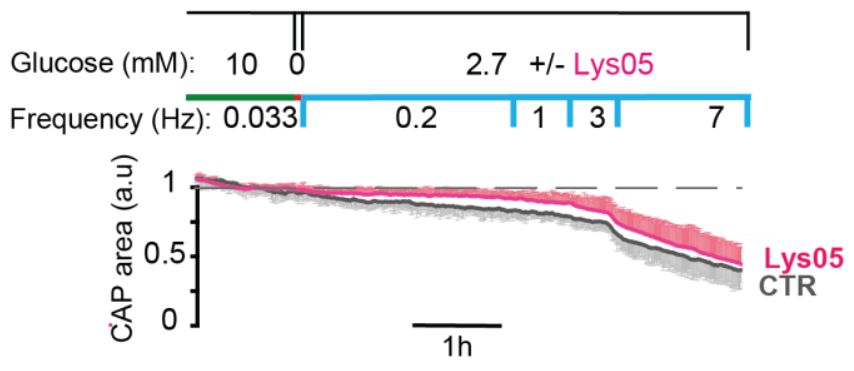

E

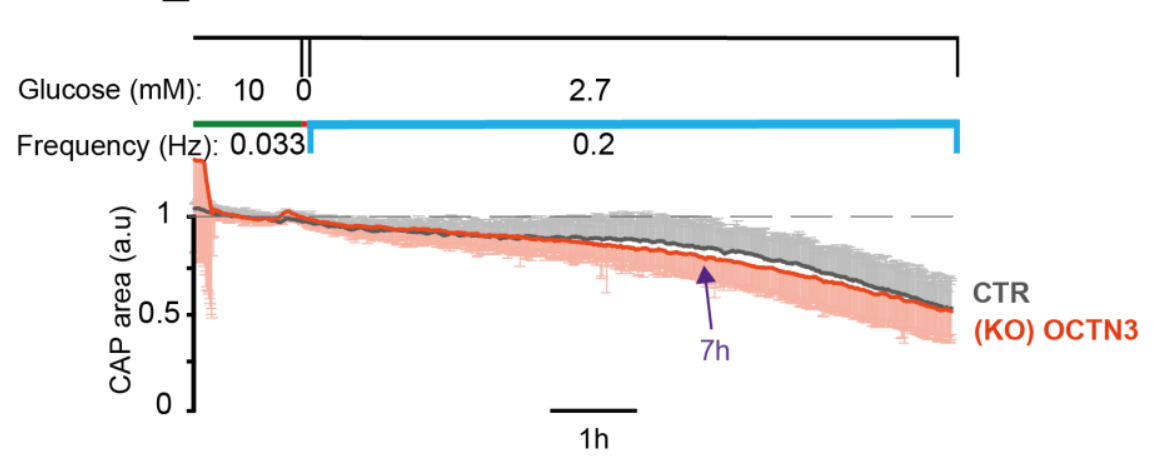

B

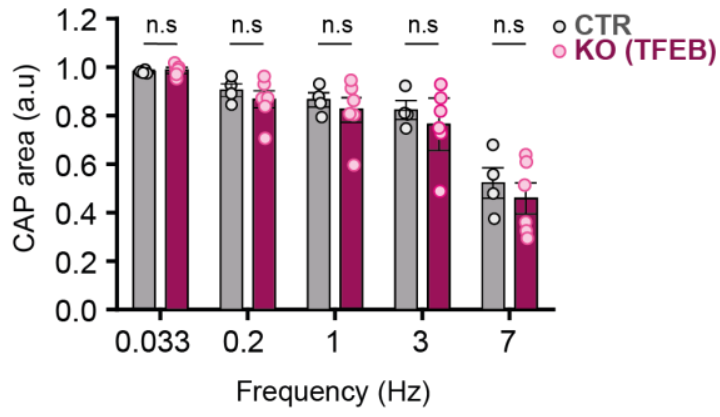

D

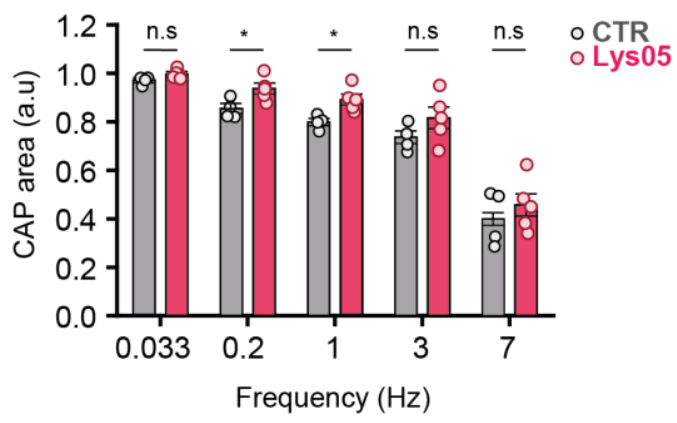

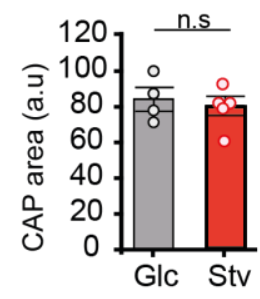

Figure 17. Effect of perturbing autophagy ((Tfeb flff * Cnp-Cre $e^{+/-}$) and pharmacologically (Lys05)) and carnitine transporter in the membrane of oligodendrocyte peroxisomes on optic nerve function under starvation.

(A) Representing the nerve function (CAP area) of optic nerve from Tfeb KO mouse line stimulated with different frequencies under starvation condition. (B) Showing the quantified CAP area (average of last $5 \mathrm{~min}$ of each frequency) presented in (A). (C) Representing the recorded CAP area from optic nerve of wild type mice stimulated with RAMP protocol under starvation condition $(2.7 \mathrm{mM}$ glucose) in the presence of autophagy inhibitor, Lys05 (5 $\mu M)$. (D) Showing the quantified CAP area (average of CAP area recorded in last $5 \mathrm{~min}$ of each frequency) for presented data in (C). (E) Showing the optic nerve function of Octn $3^{*} \mathrm{Cnp}$-Cre mouse line (KO in red and CTR in gray) recorded in the presence of $3.3 \mathrm{mM}$ glucose (stimulation frequency: $0.2 \mathrm{~Hz}$ ). (F) Representing the quantified CAP area at time point 7h (arrow head) presented in E. (t-test analysis was performed for all time point and the differences were not statistically significant). (8-9 weeks old mice, male or female, statistics: t-test, error bars: mean+/-SEM, $n \geq 6$ ). 


\subsection{Effect of glucose uptake perturbation (in vivo) in mature oligodendrocytes on myelin thickness}

\subsubsection{Confirmation of Glut1 ablation in oligodendrocytes}

In order to study the effect of starvation on myelin thickness under in vivo condition, the Glut1 $^{\mathrm{fl} / \mathrm{fl} \text { * Plp1-CreERT2 }}{ }^{+-}$(ciKO) was generated and Tamoxifen injection was performed after myelination was complete at around two months of age. Upon performing Western blot on myelin preparations from brains after around four months post tamoxifen injection, we verified the successful knock out of Glut1 in oligodendrocytes and no significant change in expression of GLUT3 and MCT1 was observed compared to the control (Fig. 18A-D). Thanks to Dr. Aiman Saab and Zoe Loser from Zurich for providing us with the samples and Dr. Kathrin Kusch in our department for doing myelin preparation and Western blot.

A

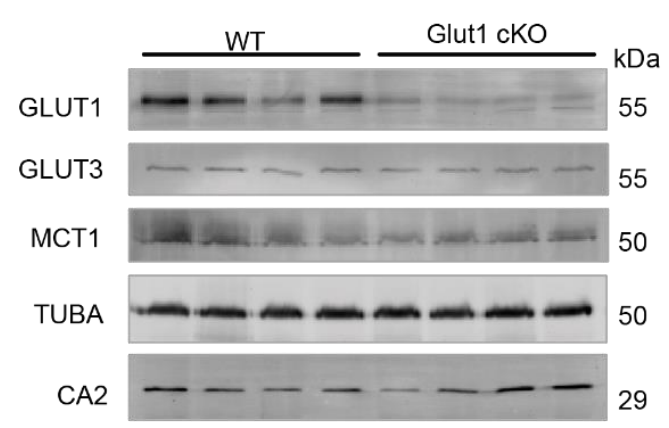

B

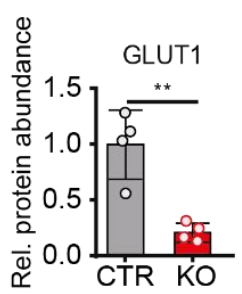

C

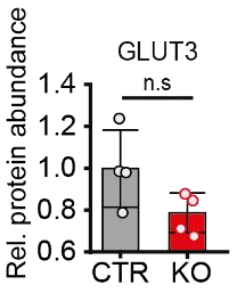

D

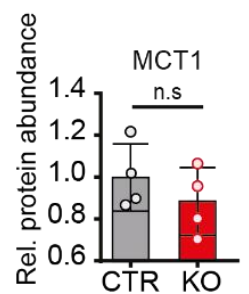

Figure 18. Immunoblotting results for myelin fraction obtained from Glut1 ciKO mice brain verifying ablation of GLUT1 transporter in oligodendrocytes.

(A) The immunoblots for GLUT1, GLUT3, MCT1, $\alpha$-tubulin (TUBA) and carbonic anhydrase2 (CA2) (TUBA and CA2 as loading controls). (B-D) quantification of GLUT1, GLUT3 and MCT1 abundance calculated by normalizing signal intensities (obtained from images recorded by the near-infrared fluorescence imager) to their corresponding total protein quantified by fast green staining and the obtained normalized data (fluorescent signal intensity/ fast green intensity) were normalized again to the mean of values calculated for control mice. (Animals: Glut1 CiKO mice four months post Tamoxifen injection, male or female, statistics: $t$-test, error bars: mean+/-SEM, n: 4).

\subsubsection{EM studies and g-ratio quantification in optic nerve of Glut1 ciKO mice}

After verifying the knocking out of the Glut1 gene in oligodendrocytes, the fixed optic nerves were subjected to electron microscopy. Thanks to our EM facility (Dr. Weibke Möbius, Torben Ruhwedel, Boguslawa Sadowski) for imaging the samples. The g-ratio quantification on 
provided images revealed the g-Ratio has increase in ciKO mice (Fig. 19A - C), implying that myelin has degraded when glucose uptake is perturbed in mature oligodendrocytes. In addition, our data revealed the inner tongue size is not significantly different in ciKO in comparison to control animals (Fig. 19D and E).

A

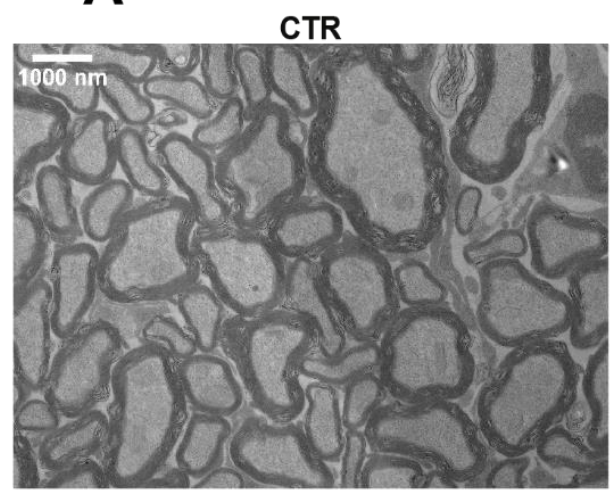

B

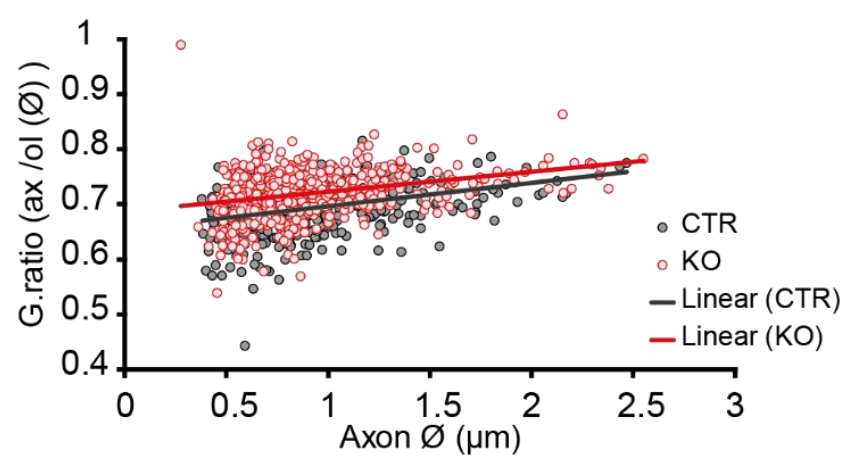

D

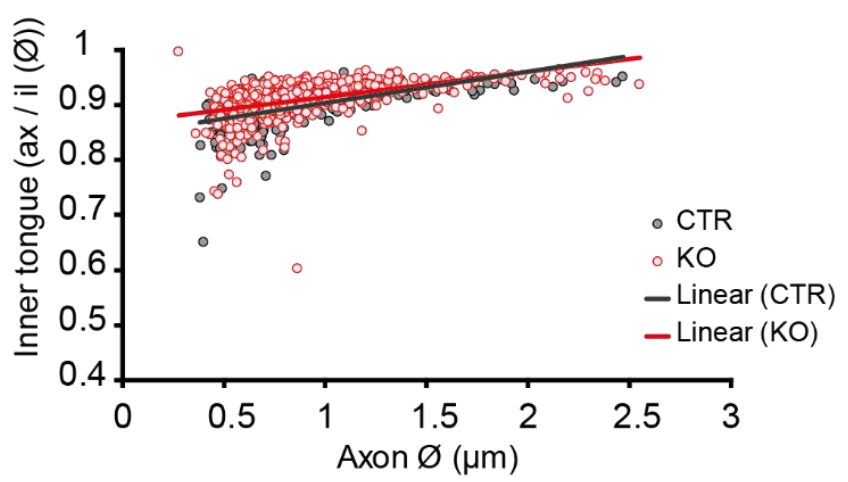

ко

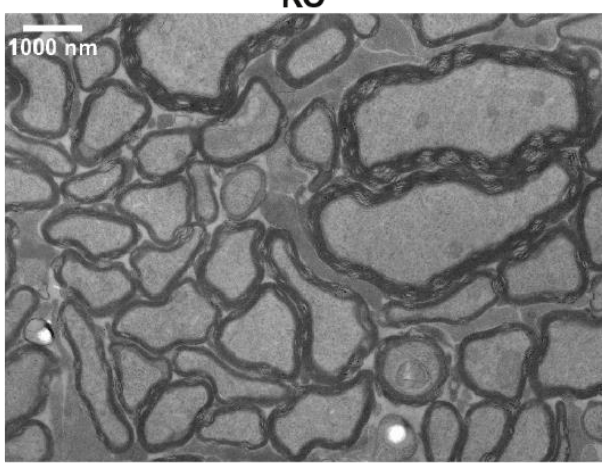

C

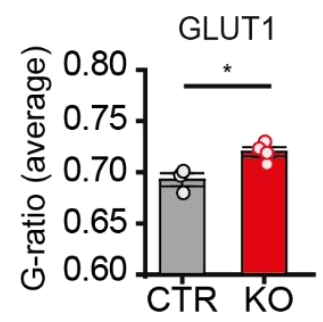

$\mathbf{E}$

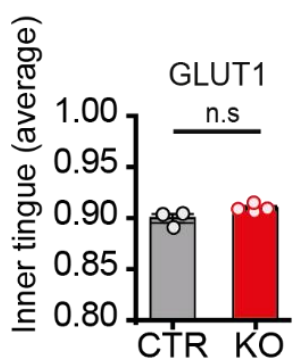

Figure 19. G-ratio quantifications for optic nerves of Glut1 ciKo mice.

(A) Electron micrographs from cross sections of the optic nerve of Glut1 ciKO mice (left and right images are corresponding to control (CTR) and knockout (KO) mice respectively). (B) calculated g-ratio (Axon (ax) $\varnothing /$ outer layer (ol) of myelin $\varnothing$ ) and their distribution in CTR and KO mice. (C) Depicting the average of g-ratio calculated for each nerve and presented as one data point in the bar graph. (D) The inner tongue changes in KO (in red) and CTR 
(in gray) calculated by dividing (Axon (ax) $\varnothing /$ inner layer (il) of myelin $\varnothing$ ). (E) Representing the average of inner tongue calculated for each nerve and presented as one data point in the bar graph. (animals: Glut1 mice four months post Tamoxifen injection, male or female, statistics: t-test, error bars: mean+/-SEM, $n \geq 3$ ). 


\section{Discussion}

\subsection{Susceptibility of different optic nerve cell types to starvation condition}

The main source of energy for the brain is glucose. However, the role of FAs in brain energy metabolism has been frequently neglected. Studies in rats have suggested that the betaoxidation of LCFAs takes place in the brain (Vignais et al., 1958) and using labeled octanoate and MRI has been estimated that FAs provide around $20 \%$ of the energy demand of the brain (Ebert et al., 2003). By employing primary cell culture from the developing rat brain it has been shown that neurons, oligodendrocytes, and astrocytes are able to utilize ketone bodies directly and that astrocytes are the only cells capable of degrading FAs (Edmond et al., 1987). It still remains unclear whether other cell types in the brain are able to oxidize FAs.

Incubation of acutely isolated MON from wild-type mice of 8-9 weeks of age, showed that the optic nerve cells can survive starvation for a long time (16h) however, astrocytes were found to be the most vulnerable cells during prolonged glucose deprivation periods (24h). In addition, applying mitochondrial beta-oxidation suggests that FAs are being used by the nerve cells under glucose shortage. Why are astrocytes dying? Among some plausible causes we can identify 1) the lack of metabolites 2) Ros formation 3) other glucose functions (e.g. pentose phosphate path). It has been shown that astrocytes are capable of producing ketone bodies trough ketogenesis (Auestad et al., 1991) and It has been suggested that under pathological condition myelin-derived FAs are transported into astrocytes and are converted to ketone bodies that support neurons (Klosinski et al., 2015). Despite this novel finding, it cannot be concluded whether the myelin-derived FAs are passed to and used by other cells to survive or whether astrocytes are releasing ketone bodies upon FA oxidation to support axons and other nerve cells under starvation condition. However, due to the fact that astrocytes are dying prior to other cells types, and other cells are still alive, it does not seem that astrocytes involve in degradation of myelin-derived FAs and releasing metabolites (such as ketone bodies) to support other cell types in the nerve. Therefore, these observations suggest that other cells in the nerve are able to oxidize FAs in the absence of astrocytes. The discrepancy between our observations with the primary cell culture results (Auestad et al., 1991). could be because of two reasons: 1) the cultured cells were obtained from immature animals and cultured cells are not $100 \%$ comparable with mature cells in-vivo condition, 2) many cell-cell interactions between optic nerve cells are absent in cell culture experiments. 
Furthermore, we could show applying $1 \mathrm{mM}$ glucose or $1.5 \mathrm{mM}$ beta-hydroxybutyrate rescue the death of astrocytes suggesting that lack of glucose that is needed for example for pentose phosphate pathway (PPP) could not be the cause of astrocytes death, rather it is suggesting probably FAs are not transported to astrocytes, since beta-hydroxybutyrate as a lipid derivative could support the survival of the astrocytes. This contradiction with the suggested mechanism by Klosinski et al (Klosinski et al., 2015) might be explained by considering other sources of FA available for astrocyte in their experiment. In our starvation experiments, the incubated nerves (ex vivo) did not have access to external sources of FAs since no FA was supplemented to aCSF and the nerve was not perfused by the blood supply, contrary to in vivo condition. In addition, recently it has been reported that neurons produce peroxidated-FA, following high neuronal activity and ROS production. These peroxidated-FAs are transported to astrocytes via ApoEpositive lipid particles for degradation (Ioannou et al., 2019). Therefore, the observed increase in FA metabolism in astrocytes could also be related to the degradation of the ApoE positive lipid particles.

To unravel the transport of FAs to astrocytes for ketogenesis purposes, further studies need to be considered. Because of the lack of a specific inhibitor for ketolysis, the use of transgenic mice (e.g. [SCOT]) should be considered. Moreover, because beta-hydroxybutyrate has been reported to play other roles other than metabolic fuel, including antioxidant properties and cellular signaling (Haces et al., 2008; Newman and Verdin, 2017), the addition of exogenous FAs would simulate the in vivo situation. Since there is evidence regarding the role of gap junctions in the transport of metabolites, applying blockers for gap junctions would also be an important experimental approach to consider in expanding our knowledge of the FA transport between cells in nervous system.

\subsection{Fatty acids are metabolized by optic nerve cells under starvation condition}

Mitochondria and peroxisomes are involved in the degradation of FAs and the interaction between these two organelles has been suggested (Baes and Van Veldhoven, 2012; Violante et al., 2013; Longo et al., 2016; Tracey et al., 2018) Inhibiting mitochondrial beta-oxidation and ketolysis resulted in the death of nerve cells under our experimental conditions (aCSF $+10 \mathrm{mM}$ glucose /or sucrose $+4-B r$; Fig. 6). However, the inhibition of peroxisomal beta-oxidation that in turn provides mitochondria with metabolic substrates, does not affect the viability of the cells when glucose is not available (aCSF + $10 \mathrm{mM}$ sucrose + Thio; Fig. 7). Given that beta- 
oxidation of VLCFAs is restricted to peroxisomes while other types of FAs are substrate for both organelles we can hypothesize a compensation for lack of peroxisomal beta-oxidation by mitochondria.

Our proteomics data suggest a shift in glucose metabolism into FA degradation during starvation. For example, there is a tendency in overexpression of hexokinase -I and II, involved in the uptake of glucose by the cell along with a down-regulation of PFKAM, the rate-limiting enzyme for glycolysis. Implying that glucose uptake and utilization for ATP production has increased and decreased respectively. These changes are accompanied by an alteration in the expression of several genes involved in FA transport and oxidation in the nerve cells. Acyl-CoA dehydrogenase, ACAD9, that plays a role in the assembly of complex I in mitochondria, apart from its dehydrogenase activity on long-chain acyl-CoA (Schiff et al., 2015) has been significantly upregulated under starvation. Crotonase, another mitochondrial enzyme involved in beta-oxidation of FAs, shows a tendency for being upregulated under starvation. Among enzymes involved in peroxisomal beta-oxidation, we have observed that one of the isoforms of thiolase (Acaala) has been significantly down-regulated however, the other isoform (Acaa1b) shows a tendency for overexpression. Since the substrate-specificity for these 2 enzymes is not well known we cannot conclude whether one isoform compensates for the other or, whether a switch between substrate utilization has taken place. FABP3 has been reported to have a direct effect on the oxidation of exogenous FAs in brown adipose tissue (Vergnes et al., 2011). Based on the Ben Barres RNA Seq database (https://www.brainrnaseq.org// this protein is expected to be highly expressed in neurons and in contrast, its lowest level of expression is expected in astrocytes. On the other hand, FABP7 with the expectation of having its highest expression in astrocytes (Ben Barres RNA seq database) has been significantly down-regulated during starvation: this appears to be in line with the conclusion that astrocytes do not seem to be receiving myelin-derived FA. In addition, in line with the fact that autophagy is induced under the condition of starvation (Glick et al., 2010), we reported the overexpression (or a tendency) of several proteins involved in autophagy, including LAMP1, LAMP2, LIMP2, RAB7A, GBRL2, and ATG3. 


\subsection{Beta-oxidation in both mitochondria and peroxisomes is involved in supporting nerve function when glucose availability is restricted}

FAs are involved in brain energy metabolism and astrocytes are considered as the only cell type capable of using FAs to produce ketone bodies. It is still a matter of debate whether 1 ) other cells of the brain, e.g. neurons, are able to utilize FAs 2) FA metabolism plays an important role in neuronal function (Schönfeld and Reiser, 2013; Panov et al., 2014; Schönfeld and Reiser, 2017). By combining ex vivo electrophysiology of young adult MON (8-9 weeks old) with confocal microscopy to measure axonal ATP via a genetically encoded ATP sensor, we provided evidence for the role of FA metabolism in neuronal function. Our data revealed that when mitochondrial beta-oxidation of FAs (and ketone bodies) is inhibited, the nerve function declines faster in comparison to untreated nerve and this decline is correlated with similar changes in axonal ATP level. Similar results were obtained when mitochondrial beta-oxidation of long-chain FAs was inhibited. Here we are providing clear evidence that the optic nerve, intended as a model for white matter tracts is capable of oxidizing FAs, as concluded via previous observations reported by Ebert et al., that estimated up to $20 \%$ of the total brain energy in rats is provided by FAs (Ebert et al., 2003). However, we should be aware that in our experiment the nerve is solely using endogenous sources of FAs stored in the nerve cells. In contrast to our observation, provided data using isolated brain mitochondria and astrocytes from rats supplemented with octanoic acid it has been concluded that the brain is not using FAs (Schönfeld and Reiser, 2017). The reasons the authors could not detect beta-oxidation are very likely because of a lack of glucose in their incubation buffer since it has been reported that FAs can be oxidized only in the presence of carbohydrates (Panov et al., 2014) or lack of cell-cell interactions. In agreement with the fact that FAs can be oxidized only in the presence of carbohydrates, we have observed in our experiments that beta-hydroxybutyrate, used as a lipid derivative can only support nerve function when glucose is available (Fig. 12), while it is well known that ketone bodies can be metabolized by the brain during starvation or during the suckling period (Owen et al., 1967; Cahill, 2006; Klosinski et al., 2015). These observations are supported by the physiological concentration of blood glucose that, even during long starvation periods never reaches zero (Jeremy M Berg, John L Tymoczko, 2002).

Applying beta-oxidation inhibitors under normal conditions (aCSF $+10 \mathrm{mM}$ glucose) did not have an impact on the nerve function, implying that the inhibitor is not toxic to the nerve. In addition, this observation might suggest that FAs do not play a role in supporting nerve function when glucose is available (in agreement with Schönfeld finding). This data should be interpreted cautiously, since applying beta-oxidation inhibitors has been reported to induce 
glucose uptake and utilization in myocytes and prostate cancer cells (Abdel-aleem et al., 1994; Schlaepfer et al., 2015) though we do not know if the same increase in glucose uptake is to be expected in nerve cells. Therefore, it is speculated that an increased glucose metabolism in nerves where beta-oxidation is inhibited might be a compensation for the lack of betaoxidation itself.

Another organelle involved in the degradation of FAs is peroxisome. Following the inhibition of peroxisomal beta-oxidation by applying pharmacology, we observed a faster decline in the compound action potential of treated nerve in comparison to control. Again, similar to mitochondrial beta-oxidation, the inhibition of peroxisomal beta-oxidation affected the ATP level in the axons. This observation is in contrast with our data obtained from optic nerve incubation experiment showing that peroxisomal beta-oxidation inhibition does not induce cell death under starvation. This discrepancy in our observation can be explained by considering the energy demand differences under starvation, between housekeeping activities and the function of the nerves. In our incubation experiment, we revealed optic nerve cells can survive for long time (16h) in the absence of glucose while based on electrophysiology recordings the nerve cannot maintain its function (under the same condition) for more than 15 min during which stored glycogen in astrocytes can still support the nerve function (Brown et al., 2003). Suggesting that peroxisomal beta-oxidation becomes essential when energy demand is higher. The recorded nerve function under normal condition vs. in the presence of peroxisomal beta-oxidation inhibition excludes a toxic effect of the inhibitor and shows that the observed decline under starvation is rather energy-dependent.

\subsection{Beta-oxidation in myelin peroxisomes play an important role in supporting optic nerve function under starvation}

By ectopically applying specific drugs, beta-oxidation can be inhibited, however this is a global effect that affects all cells. By using conditional KO mice $\left(M f p 2^{f / / f l} * C n p-C r e^{+/}\right)$we could observe an important role for peroxisomes in oligodendrocytes in supporting nerve function, under metabolic stress. The $\mathrm{Mfp}^{\mathrm{fl} / \mathrm{fl}}$ * $\mathrm{Cnp}-\mathrm{Cr} e^{+/-}$mice have perturbed peroxisomal beta-oxidation specifically in oligodendrocytes and also in myelin processes: this line has been previously described by Verheijden et al. and the authors reported no phenotype or obvious abnormality in myelin structure, at least in mice younger than 12 months. The implication is that peroxisomal beta-oxidation seems not crucial for myelin development (Verheijden et al., 2013). 
Therefore, the observed decline during our experimental paradigm cannot be explained by pre-existing damage to the nerve given that our experimental mice were very young (8-9 weeks old).

The obtained data from nerve conduction recording clearly shows that FA beta-oxidation, both in peroxisomes and mitochondria, are involved in supporting nerve function under metabolically challenging conditions. However, which cells are degrading FAs remains unclear. There are two possible scenarios for the fate of FAs in white matter tracts exposed to starvation: 1) FAs can be used/preferred by glial cells under starvation for ATP production: the consequence of this is the saving of glucose (or derivatives) that can be transported directly to neurons for supporting the nerve function 2) FAs (or derivatives) are transported directly into axons for complete degradation to $\mathrm{CO}_{2}$ and ATP production in axonal mitochondria. The results obtained from the nerve incubation in the absence of glucose suggests that FAs (derivatives) are metabolized by glial cells under starvation. For verifying the second scenario, i.e. the oxidation of FAs and derivatives directly by axonal mitochondria should be further clarified. It is well accepted that lipid-derived ketone bodies can support the brain function: using rat brain neuronal culture it has been shown that neurons can, in fact, oxidize ketone bodies (Edmond et al., 1987). Using isolated mitochondria from neurons it has been reported that palmitate could be fully oxidized by axonal mitochondria to $\mathrm{CO}_{2}$ (Panov et al., 2014). On the other hand, the long-chain FA are considered uncoupling agents (Takeuchi et al., 1991) and can also affect the electron transport chain in mitochondria, resulting in the production of reactive oxygen species (Cocco et al., 1999; Loskovich et al., 2005; Schönfeld and Wojtczak, 2007). Therefore, beta-oxidation of FAs in neuronal mitochondria can lead to the generation of reactive oxygen species, particularly harmful to neurons. In addition, beta-oxidation of FA consumes a higher amount of oxygen in comparison to glucose (or its derivatives): this increases the risk of a hypoxic condition for neurons. Because of the mentioned side effects of FA utilization for ATP production, the brain "prefers" glucose over FA as a primary source of energy (Schönfeld and Reiser, 2013). Moreover, the expression of carnitine palmitoyl transferase (CPT1a and CPT1b), necessary for the uptake of long-chain FA by mitochondria, is very low in neurons and restricts beta-oxidation to long-chain FA in neurons (Cahoy et al., 2008; Lee and Wolfgang, 2012). It should be noted that CPT1c is expressed specifically in neurons and plays an important role in energy homeostasis, however, its enzymatic activity is yet not clear and it does not support FA oxidation (Wolfgang, 2016) (Roa-Mansergas et al., 2018). Considering the interaction between mitochondria and peroxisomes and low level of glucose, the above-mentioned restrictions are 
explainable: peroxisomes are accumulated in the paranodal regions, the active zone of axonmyelin interaction (Kassmann, 2014). These paranodal peroxisomes do not seem to be trapped during myelin compaction since their presence has been reported in cytoplasmic channels, stretches of oligodendrocyte's cytoplasm that connect with the paranodal loops (Kassmann et al., 2011) Therefore, it is speculated that the presence of myelin peroxisomes near axonal mitochondria should serve some metabolic function (Kassmann, 2014). Since our electrophysiology data shows myelin peroxisomal beta-oxidation is involved in supporting nerve function, it suggests peroxisomes providing metabolites to axonal mitochondria. Considering that one of the aims for beta-oxidation is the chain shortening of FAs, the end products, medium and short-chain FAs, e.g. propionyl-CoA (Wanders et al., 2016), acetyl- CoA, acetate, and acetoacetyl-CoA (Hovik et al., 1991) are very likely transported into axonal mitochondria for ATP production. Since these metabolites are shortened in their FA carbonbackbone, they do not result in uncoupling of the ETC in the inner mitochondrial membrane (Schönfeld and Wojtczak, 2016). In addition, medium and short-chain FA do not need FABP for their intracellular trafficking and their import into mitochondria is independent of the carnitine palmitoyl-transferase system (CPT1a and b). These possible explanations are supported by evidence of an accumulation of lipid droplets in glial cells observed in a CPT2- knockout in drosophila. CPT2 rescue only in glial cells was sufficient for the degradation of these lipid droplets and it is suggested that the produced ketone bodies are then used for energy production (Schulz et al., 2015). These results lead to the conclusion that when glucose concentration is transiently low, e.g. under starvation, the oxygen demand for the degradation of FA is temporarily elevated, without detrimental effects on neurons.

In conclusion, the interaction between myelin and axonal mitochondria, preserved in ex-vivo and in-vivo preparations, seems to make the degradation of FA (derivatives) by neurons logically possible (such interaction is missed in cell culture experiments) and further investigations using $\mathrm{KO}$ mice with neuron-specific perturbation of lipid and ketone body metabolism is required to define which type of FA derivatives are used possibly by neurons.

\subsection{Investigation on mechanism of myelin degradation and export of peroxisomal beta-oxidation products}

Autophagy is induced under starvation to provide metabolites for energy production and TFEB has been reported as a regulator of autophagy and lipophagy (Settembre and Ballabio, 2014). 
Based on RNA-seq data, this transcription factor is expressed in myelinating oligodendrocytes as well (https://www.brainrnaseq.org/). In order to see if autophagy is induced in oligodendrocytes to release myelin-derived FAs for beta-oxidation, optic nerves from Tfeb KO mice were subjected to electrophysiology. However, the nerve function was not significantly different between CTR and KO mice. These observations suggest that TFEB does not play an important role in releasing FAs in oligodendrocytes under starvation conditions. However, it does not exclude the role of autophagy in myelin digestion. It has been reported recently that the expression of lysosomal and autophagy genes and the number of LAMP1-positive puncta

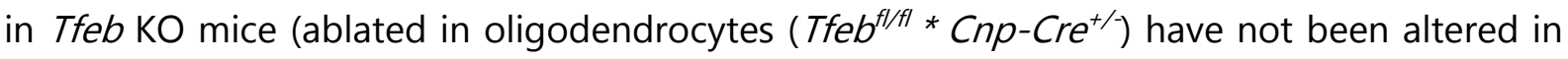
comparison with CTR. A recent study suggests other autophagy regulators such as TFE3 might function redundantly or cooperatively with TFEB (Sun et al., 2018). Since other autophagy regulators might compensate for lack of TFEB, it seems that the necessary machinery for autophagy is still present in oligodendrocytes even in the absence of TFEB. This might explain why the nerve function is not affected in Tfeb KO mice under starvation conditions. On the other hand, applying autophagy inhibitors suggest that autophagy is not involved in myelin degradation. This discrepancy might be due to the insufficient dose of inhibitor applied to block autophagy. Therefore, these findings remain inconclusive at this stage and further experiments should be done for unraveling the underlying mechanism of myelin degradation.

It has been reported that OCTN3 might be involved in the export of beta-oxidation products from peroxisomes (Januszewicz et al., 2009). To see if OCTN3 plays important role in axonal support by myelin peroxisomes, OCTN3 was ablated specifically in oligodendrocytes (Octn $3^{f / f f}$ * $\mathrm{Cnp}-\left(\mathrm{Cr} \mathrm{e}^{+/}\right)$and recording of the nerve function was performed. Our data did not show any significant difference between nerve function of CTR and KO mice under starvation conditions suggesting either OCTN3 is not involved in supporting nerve function or that OCTN3 functions with other possible transporters cooperatively or redundantly. Another possibility is that the end products of peroxisomal beta oxidation are not exported in carnitylated form. Since the mechanism of metabolite transport across peroxisomal membrane is largely unknown, this remains unanswered and further investigation is needed for unraveling the mechanism of metabolite transport into axons. 


\subsection{Myelin: a source of energy when glucose is limited}

As opposed to prolonged starvation, under a "fed condition" the organism's adipose tissue can store lipids within a single, large lipid droplet that can occupy the cell cytoplasm almost entirely (Nishimoto and Tamori, 2017). This lipid structures have been described in the brain of unmyelinated species like Lamprey (Weil et al., 2018) and evidence for their role in axon-glia metabolic coupling has been provided in drosophila in a study that speculated a possible role for FA metabolism in aging and Alzheimer disease (Schulz et al., 2015). However, until now there is no report on the presence of lipid droplets in oligodendrocytes. To study the effect of starvation on myelin in vivo we employed a Glut1 ciKO (G/ut1 $1^{f / f l} *$ Plp1-CreERT2 $\left.{ }^{+/}\right)$. Glucose uptake via Glut1 is therefore perturbed in post-myelinating oligodendrocytes and the measured g-ratio as a readout for myelin thickness showed a decrease in myelin thickness in the optic nerves [19]. This observation implies that myelin-derived FAs can be degraded under a shortage of glucose. Importantly, although the myelin was found to be thinner in knock-out mice at four months post tamoxifen injection, the animals did not show any obvious phenotype, or neuroinflammation. However, at present, we cannot exclude the possibility of neurodegeneration shortly after tamoxifen injection. The lack of phenotype in Glut1 ${ }^{f / f l} *$ Plp1$\mathrm{CreERT2}^{+/-}$mice with thinner myelin is unexpected since myelin thickness is important for the conduction velocity of action potential propagation and consequently affects the neuronal signal processing. Similar results were obtained by incubating acutely isolated wild-type optic nerves under glucose deprivation. Altogether these observations suggest a new function for myelin, as an energy reservoir: a function similar to the lipid droplets as a store of energy during metabolic stress and substrate shortage. The maintenance of a close-to-normal brain function while a thinner myelin is present can be explained by changes in nodal length as a mechanism that has been predicted by modelling to be energy saving (Arancibia-Cárcamo et al., 2017). Our observations supported by several studies showing changes in white matter (under specific conditions). In anorexia nervosa, a severe mental disorder in which energy intake is restricted persistently leading to a significant body weight loss, alterations in white matter microstructure of the corpus callosum and possible myelin loss have been described(Swayze et al., 2003) (Nickel et al., 2019). Along with these findings, it has been reported that during aging and Alzheimer's disease, a decrease in glucose uptake is accompanied by a metabolic shift toward FA oxidation where myelin is being degraded to fuel brain cells. However, in this study, myelin degradation was observed under pathological conditions and the authors could not conclude to which extent such myelin degradation can occur in the healthy brain. Taken together, we propose that the energy-rich FAs contained in myelin act as an energy storage and can protect 
neurons when glucose availability is transiently (not persistent like pathological condition) scarce and might explain the importance of myelin in neurodegenerative diseases (Fig. 20).

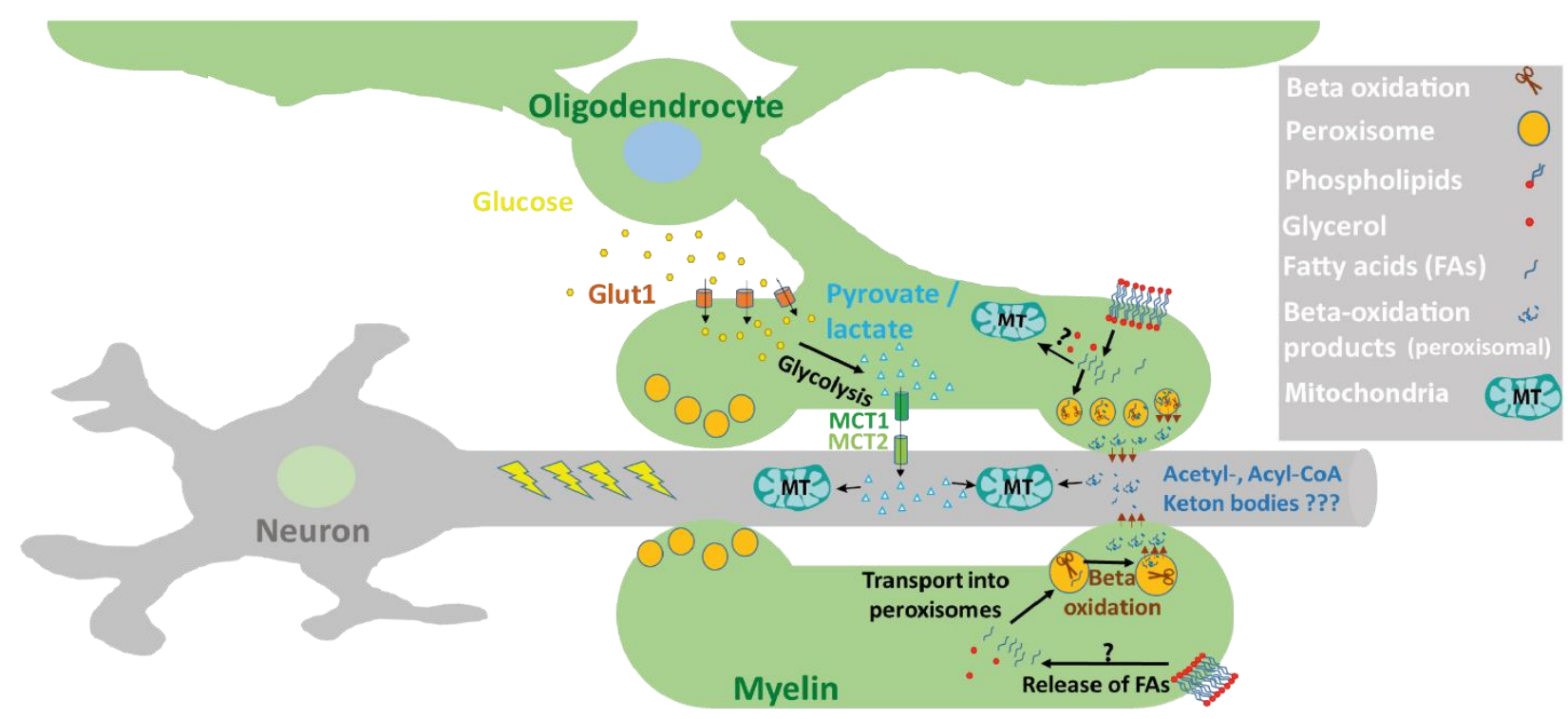

Figure 20. Interaction between myelin peroxisomes and mitochondria in degradation of myelin FA under starvation.

Myelin-derived FAs are released under metabolic stress condition and depending on their chain length they could be directly degraded in mitochondria or after going through peroxisomal beta-oxidation, the beta oxidation products could be used for supporting neuronal function. The exact mechanism by which FAs are released and transported remains unknown. 


\section{Material and methods}

\subsection{Mouse lines}

All animals were breeded in the animal facility of the Max- Planck Institute for Experimental Medicine (MPI-EM) and were kept under a $12 \mathrm{~h}$ day and night cycle with access to food and water ad libitum. All the experiments were carried out in compliance with approved animal policies of the MPI-EM. In this work the following mouse lines were used:

\subsubsection{ALDG mouse line (mixed background)}

The Aldh-GFP mouse line expresses GFP specifically in astrocytes. In this mouse line enhanced green fluorescent protein (EGFP) is expressed under aldehyde dehydrogenase 1 family, member L1 (Aldh1l1). This mouse line was generated provided by Dr. Gesine Saher (http://www.informatics.jax.org/allele/MGl:3843271). The background of this mouse line was originally Swiss albino and the animals were cross breeded with C57BI6 mice and resulting offspring with mixed background were used in our experiments.

\subsubsection{Cxcr-GFP mouse line (C57BL6 background)}

This mouse line was present in the animal facility of MPI-EM expresses GFP under a Cxcr promoter and specifically labels microglia (Jung et al., 2000).

\subsection{3. mEos2 mouse line (C57BL6 background)}

The mEos2 mouse line has labeled oligodendrocyte. In this mouse line the mEos2 (CnpmEos2-PTS1) is expressed under Cnp1 promoter and because of the presence of targeting signal sequence for peroxisomes (PTS1), this fluorophore is transported into peroxisomes of oligodendrocytes (Richert et al., 2014). 


\subsubsection{Ng2-YFP mouse line (C57BL6 background)}

The Ng2-YFP mice express yellow fluorescent protein under the NG2 promoter such that oligodendrocyte precursor cells (OPCs) are labeled specifically (Karram et al., 2008). This mouse line was used for distinguishing oligodendrocyte precursor cells from other cell populations in the mouse optic nerve.

\subsubsection{Mfp2 mouse line (C57BL6 background)}

Mfp2 ${ }^{f / f t}$ transgenic mice generously provided by Prof. Myriam Baes (Verheijden et al., 2013), were cross breed with Cnp-Cre mouse line (Lappe-Siefke et al., 2003) to generate conditional knock out mice (Mfp2 $\left.{ }^{f / f t} * \mathrm{Cnp}-\mathrm{Cre}^{+/-}\right)$(Thanks to Sarah Richert) with perturbed peroxisomal beta-oxidation in oligodendrocytes. In this project, the optic nerve from this mouse line was used for studying the role of peroxisomal beta-oxidation in supporting nerve function under starvation condition.

\subsubsection{Glut1 mouse line (C57BL6 background)}

Glut1 ${ }^{f / f l}$ mice was a generous gift from Prof. E. Dale Abel (Iowa university) has been described previously (Young et al., 2011). In order to generate ciKO mice, they were cross breed with Plp1-CreERT2 mice (Leone et al., 2003) in the animal facility of MPI-EM. The generated Glut1 ${ }^{f / f l} *$ Plp1-CreERT2 were used for the experiments.

\section{Tamoxifen injection:}

In order to exclude the developmental effects of lack of GLUT1 protein in oligodendrocytes, the animals were injected with Tamoxifen at two months of age, when myelination is complete. Recombination of Glut1 in oligodendrocytes in ciKO animals was induced by injecting mice with Tamoxifen. Tamoxifen (Sigma Aldrich) was dissolved in corn oil at $10 \mathrm{mg} / \mathrm{ml}$ concentration. At around two months of age, when myelination is complete, the 
animals were injected intraperitoneally (IP) with a dose of $100 \mathrm{mg} / \mathrm{kg}$ bodyweight/day of Tamoxifen for five consecutive days and the tissues were collected four months after injection.

\subsubsection{Tfeb mouse line (C57BL6 background)}

Previously described transgenic $T f e b^{f / f l}$ mice were generously provided by Prof. Andrea Blabio (Settembre et al., 2012). This mouse line was cross breed with Cnp-Cre mouse line (Lappe-Siefke et al., 2003) and the conditional knock out mice ( $\mathrm{Tfe}^{\mathrm{fl} / \mathrm{fl}}$ * $\mathrm{Cnp}-\mathrm{Cr} \mathrm{f}^{+/}$) were generated in the animal facility of Max Planck Institute for Experimental Medicine.

\subsubsection{Octn3 mouse line (C57BL6 background)}

To generate Octn3 conditional knockout mice, the sperm of Octn $3^{f / f f}$ mice $\left(S / c 22 a 21^{\text {tm1a(KOMP)Wtsi }}\right)$ was ordered from KOMP. The ordered sperms were used for IVF to generating Octn $3^{/ 0 x P / w t}$ mice. Generated $O c t n 3^{f / f l}$ mice were cross breeded with Cnp-Cre mouse line (Lappe-Siefke et al., 2003) to specifically knock out OCTN3 in oligodendrocytes. Generated $\mathrm{KO}$ mice $\left(\mathrm{Octn}^{\mathrm{fl} / f l} * \mathrm{Cnp}-\mathrm{Cr} \mathrm{e}^{+/}\right)$were used in this project.

\subsubsection{THYATPA mouse line (C57BL6 background)}

In order to monitor ATP level in axons, the THYATPA mouse line with a genetically encoded ATP sensor was used. The animals generously were provided by Prof. Johannes Hirrlinger and Dr. Andrea Trevisiol. The mouse line as described previously, expresses ATPsensor ATeam1.03 ${ }^{\text {YEMK }}$ in neurons under control of Thy1.2 promoter (Trevisiol et al., 2017). 


\subsection{Genotyping}

\subsubsection{DNA extraction}

The Nexttec ${ }^{\text {TM }}$ Tissue $\&$ Cells kit was used for DNA extraction from ear or tail biopsies for genotyping the animals via PCR. DNA preparation was performed according to the

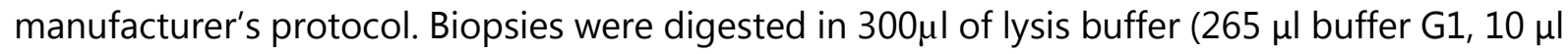
buffer $\mathrm{G} 2,25 \mu \mathrm{l}$ buffer $\mathrm{G} 3$ ) by shaking for $1-2 \mathrm{~h}$ at $62{ }^{\circ} \mathrm{C}$. The columns were prepared by equilibrating the NexttecTM cleanPlate96 with $350 \mu$ l Prep buffer for $5 \mathrm{~min}$ at TR and centrifugation for $1 \mathrm{~min}$ at $350 \mathrm{~g}$. In next step, $120 \mu \mathrm{l}$ of the lysates were loaded onto equilibrated columns and after 3 min incubation at RT, the DNA was collected into fresh tubes by $1 \mathrm{~min}$ centrifugation at $750 \mathrm{~g}$. In final step, the DNA obtained from tail and ear biopsies were diluted to $1: 8$ and $1: 3$ respectively and used for genotyping.

\subsubsection{PCR reaction}

Polymerase chain reaction (PCR) is used for amplification of a DNA sequence of interest (template DNA) in vitro (Mullis et al., 1986). The PCR cycles composed of three steps including denaturation, annealing, and extension of the DNA template sequence. During denaturation step, because of the high temperature the template DNA and primers (short sequences of DNA with around 18-30bp that are complementary to specific sequences of the gene of interest) are in single stranded form. In the second step, by lowering the temperature, the primers bind to the template DNA and provides the free $3^{\prime}$ end for acting a thermostable DNA polymerase, Taq polymerase. In last step, the polymerase synthesizes the DNA of interest by incubation at around $72^{\circ} \mathrm{C}$, the optimal temperature for Taq polymerase activity. Repeating this three-step cycle for several times results in amplification of the sequence of interest. 


\subsubsection{TAE buffer for gel electrophoresis}

50 x Tris-acetate EDTA (TAE) Buffer:

$\begin{array}{lll}50 \mathrm{mM} & & \text { EDTA } \\ 2.0 \mathrm{M} & & \text { Tris-acetate, } \quad \mathrm{pH}: \\ & 8.0 & \\ 57.1 \mathrm{ml} & & \text { glacial acid }\end{array}$

Fill up to 1 l with ddH2O

\subsubsection{Agarose gel electrophoresis}

The PCR products were separated on $2 \%$ agarose in TAE buffer (W/V). Briefly, agarose was added to TAE (1x) buffer and heated till agarose was completely dissolved. The prepared agarose solution was poured into a gel casting chamber with placed combs in appropriate distances. After gel formation (1-2 h at RT), the combs were removed gently and created wells were used for loading the PCR products. The gel was transferred into an electrophoresis chamber filled with TAE buffer $(1 x)$ and the mixed PCR products with GelRed (1:5 from stock 1:4000) were loaded. In order to estimate the size of the products, an appropriate DNA marker was also loaded next to samples. By applying appropriate voltage, the negatively charged DNA fragments start to move toward the positively charged electrode and being separated based on their size. In final step, the separated fragments of DNA were visualized under UV light.

\subsubsection{Genotyping primers and PCR protocols}

All the genotyping primers listed below were synthesized at the 'DNA core facility' of the Max-Planck Institute of Experimental Medicine and the following protocols were applied for performing PCR: 


\section{Aldg genotyping PCR:}

\section{Primers for Aldg}

Sense primer:

5'-CCCTTTCTAACCCTCTGGC -3'

Antisense primer:

5'-TCCTTGAAGAAGATGGTGCG -3'

PCR master mix for GoTaq polymerase

$1.00 \mu \mathrm{l}$

$0.10 \mu \mathrm{l}$

$0.10 \mu \mathrm{l}$

$2.00 \mu \mathrm{l}$

$4.00 \mu \mathrm{l}$

$0.15 \mu \mathrm{l}$

$12.65 \mu \mathrm{l}$

$$
\text { DNA (100 pg-100 ng) }
$$
sense primer $(50 \mathrm{pM})$

antisense primer (50 pM)

dNTP mix (2 mM)

5x GoTaq buffer

GoTaq polymerase $(5 \mathrm{U} / \mu \mathrm{l})$

$\mathrm{ddH}_{2} \mathrm{O}$
PCR program

$95^{\circ} \mathrm{C}$

$3 \mathrm{~min}$

$\left\{\begin{array}{l}51^{\circ} \mathrm{C} \\ 72^{\circ} \mathrm{C} \\ 95^{\circ} \mathrm{C}\end{array}\right.$

$50^{\circ} \mathrm{C}$

$72^{\circ} \mathrm{C}$

$4^{\circ} \mathrm{C}$

$30 \mathrm{sec}$ $40 \mathrm{sec} \times 34$

$15 \mathrm{sec}$

$1 \mathrm{~min}$

$10 \mathrm{~min}$

pause

\section{Cxcr-Gfp genotyping PCR:}

\section{Primers for Cxcr-Gfp}

Sense primer:

Sense primer:

Antisense primer:
5'-TCAGTGTTTTCTCCCGCTTGC -3'

5'-GTAGTGGTTGTCGGGCAGCAG -3'

5'-CAGTGATGCTCTTGGGCTTCC -3'
PCR master mix for GoTaq polymerase

$1.00 \mu \mathrm{l}$

$0.1 \mu l$

$0.05 \mu \mathrm{l}$

$0.15 \mu \mathrm{l}$

$2.00 \mu \mathrm{l}$

$4.00 \mu \mathrm{l}$

$0.1 \mu l$

$12.6 \mu \mathrm{l}$
DNA (100 pg-100 ng)

sense primer (50 pM)

sense primer (50 pM)

antisense primer (50 pM)

dNTP mix (2 mM)

5x GoTaq buffer

GoTaq polymerase $(1 \mathrm{U} / \mu \mathrm{l})$

dd $\mathrm{H} 2 \mathrm{O}$
PCR program $95^{\circ} \mathrm{C}$

$50^{\circ} \mathrm{C}$

$72^{\circ} \mathrm{C}$

$95^{\circ} \mathrm{C}$

$50^{\circ} \mathrm{C}$

$72^{\circ} \mathrm{C}$

$4^{\circ} \mathrm{C}$
$3 \mathrm{~min}$

$30 \mathrm{sec}$

1 min

$30 \mathrm{sec}$

$1 \mathrm{~min}$

$10 \mathrm{~min}$

pause 
mEos2 genotyping PCR:

\section{Primers for $m E$ Eos2}

Sense primer:

5'- CTTCTTACACAGGCCACCATGAGTGCG-3'

Antisense primer:

5'-GGATCCTTACTTAGTTAAAGCTTGGATCGT-3'

PCR master mix for GoTaq polymerase

$1.00 \mu \mathrm{l}$

$0.10 \mu \mathrm{l}$

$0.10 \mu \mathrm{l}$

$2.00 \mu \mathrm{l}$

$4.00 \mu \mathrm{l}$

$0.15 \mu \mathrm{l}$

$12.65 \mu \mathrm{l}$

$$
\begin{gathered}
\text { DNA (100 pg-100 ng) } \\
\text { sense primer }(50 \mathrm{pM}) \\
\text { antisense primer }(50 \mathrm{pM}) \\
\text { dNTP mix (2 mM) } \\
5 x \text { GoTaq buffer } \\
\text { GoTaq polymerase }(5 \mathrm{U} / \mu \mathrm{l})
\end{gathered}
$$

$\mathrm{ddH}_{2} \mathrm{O}$

\section{PCR program}

$3 \mathrm{~min}$

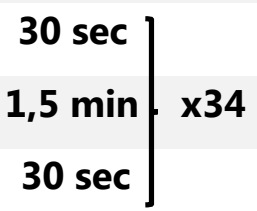

$1 \mathrm{~min}$

$10 \mathrm{~min}$

$72^{\circ} \mathrm{C}$

$4^{\circ} \mathrm{C}$

\section{Ng2-YFP genotyping PCR:}

\section{Primers for Ng2-YFP}

Sense primer:

Sense primer:

Antisense primer:

\begin{tabular}{|c|c|c|c|c|}
\hline $1.00 \mu \mathrm{l}$ & DNA (100 pg-100 ng) & $95^{\circ} \mathrm{C}$ & $3 \mathrm{~min}$ & \multirow{4}{*}{ x34 } \\
\hline $0.1 \mu l$ & sense primer (50 pM) & $50^{\circ} \mathrm{C}$ & $30 \mathrm{sec}$ & \\
\hline $0.1 \mu \mathrm{l}$ & sense primer (50 pM) & $72^{\circ} \mathrm{C}$ & $1 \mathrm{~min}$ & \\
\hline $0.1 \mu l$ & antisense primer (50 pM) & $95^{\circ} \mathrm{C}$ & $30 \mathrm{sec}$ & \\
\hline $2.00 \mu \mathrm{l}$ & $\mathrm{dNTP} \operatorname{mix}(2 \mathrm{mM})$ & $50^{\circ} \mathrm{C}$ & $1 \mathrm{~min}$ & \\
\hline $4.00 \mu \mathrm{l}$ & 5x GoTaq buffer & $72^{\circ} \mathrm{C}$ & $10 \mathrm{~min}$ & \\
\hline $0.1 \mu \mathrm{l}$ & GoTaq polymerase $(1 \mathrm{U} / \mu \mathrm{l})$ & $4^{\circ} \mathrm{C}$ & pause & \\
\hline $12.6 \mu \mathrm{l}$ & $\mathrm{ddH}_{2} \mathrm{O}$ & & & \\
\hline
\end{tabular}

5'-CGCTGAACTTGTGGCCGTTTA -3'

5'-ACAGCTTTCCTTCCAGAC -3'

5'-TGACCTTGGATTCTGAGC -3'

PCR master mix for GoTaq polymerase

PCR program 
Mfp2 genotyping PCR:

\section{Primers for $\mathbf{M f p 2}$}

Sense primer:

5'-CCCAACGCTGGGTCACGGATGACGG-3'

Antisense primer:

5'-GCAACCATAAGTTACACAAAATGCC-3'

PCR master mix for GoTaq polymerase

PCR program

\begin{tabular}{|c|c|c|c|c|}
\hline $1.00 \mu \mathrm{l}$ & DNA (100 pg-100 ng) & $95^{\circ} \mathrm{C}$ & $3 \mathrm{~min}$ & \\
\hline $0.10 \mu \mathrm{l}$ & sense primer $(50 \mathrm{pM})$ & $65^{\circ} \mathrm{C}$ & $1 \mathrm{~min}$ & \\
\hline $0.10 \mu \mathrm{l}$ & antisense primer (50 pM) & $72^{\circ} \mathrm{C}$ & $1 \mathrm{~min}$ & x35 \\
\hline $2.00 \mu \mathrm{l}$ & $\mathrm{dNTP} \operatorname{mix}(2 \mathrm{mM})$ & $95^{\circ} \mathrm{C}$ & $1 \mathrm{~min}$ & \\
\hline $4.00 \mu \mathrm{l}$ & 5x GoTaq buffer & $60^{\circ} \mathrm{C}$ & $1 \mathrm{~min}$ & \\
\hline $0.1 \mu \mathrm{l}$ & GoTaq polymerase $(1 \mathrm{U} / \mu \mathrm{l})$ & $72^{\circ} \mathrm{C}$ & $10 \mathrm{~min}$ & \\
\hline $12.7 \mu \mathrm{l}$ & $\mathrm{ddH}_{2} \mathrm{O}$ & $4^{\circ} \mathrm{C}$ & pause & \\
\hline
\end{tabular}

Cnp-Cre genotyping PCR:

Primers for Cnp-Cre

Sense primer:

5'- GCCTTCAAACTGTCCATCTC-3'

Sense primer:

5'- CATAGCCTGAAGAACGAGA-3'

Antisense primer:

5'- CCCAGCCCTTTTATTACCAC-3'

PCR master mix for GoTaq polymerase

PCR program

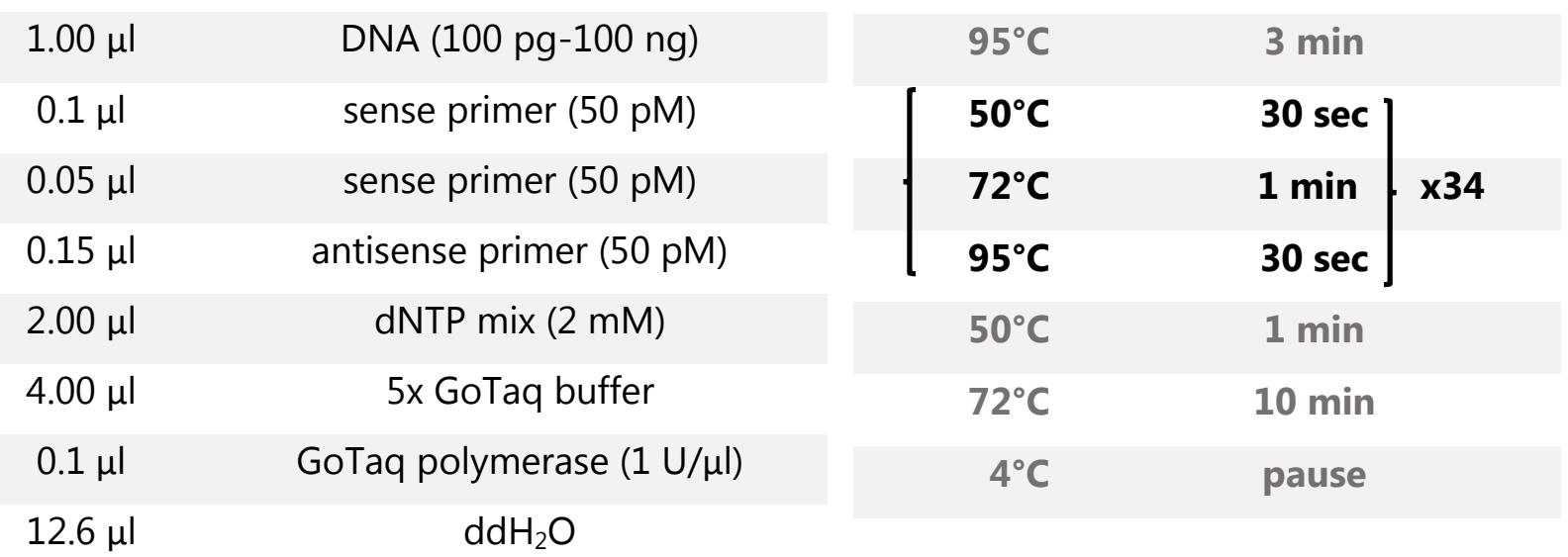




\section{Glut1 genotyping PCR:}

\section{Primers for Glut1}

Sense primer:

5'-CTGTGAGTTCCTGAGACCCTG -3'

Antisense primer:

5'-CCCAGGCAAGGAAGTAGTTC -3'

PCR master mix for GoTaq polymerase

$1.00 \mu \mathrm{l}$

$0.10 \mu \mathrm{l}$

$0.10 \mu \mathrm{l}$

$2.00 \mu \mathrm{l}$

$4.00 \mu \mathrm{l}$

$0.1 \mu \mathrm{l}$

$12.7 \mu \mathrm{l}$
DNA (100 pg-100 ng) sense primer $(50 \mathrm{pM})$

antisense primer (50 pM)

dNTP mix (2 mM)

5x GoTaq buffer

GoTaq polymerase $(1 \mathrm{U} / \mu \mathrm{l})$

$\mathrm{ddH}_{2} \mathrm{O}$
PCR program

$95^{\circ} \mathrm{C}$

3 min

$\left\{\begin{array}{l}60^{\circ} \mathrm{C} \\ 72^{\circ} \mathrm{C} \\ 95^{\circ} \mathrm{C}\end{array}\right.$

$60^{\circ} \mathrm{C}$

$72^{\circ} \mathrm{C}$

$4^{\circ} \mathrm{C}$

$30 \mathrm{sec}$

$45 \mathrm{sec} \times 30$

$30 \mathrm{sec}$

1 min

$10 \mathrm{~min}$

pause

\section{Pcet genotyping PCR:}

\section{Primers for Pcet}

Sense primer:

Antisense primer:
5'-TGGACAGCTGGGACAAAGTAAGC -3'

5'-CGTTGCATCGACCGGTAATGCAGGC -3'
PCR master mix for GoTaq polymerase

$\begin{array}{cc}1.00 \mu \mathrm{l} & \text { DNA (100 pg-100 ng) } \\ 0.10 \mu \mathrm{l} & \text { sense primer (50 pM) } \\ 0.10 \mu \mathrm{l} & \text { antisense primer (50 pM) } \\ 2.00 \mu \mathrm{l} & \text { dNTP mix (2 mM) } \\ 4.00 \mu \mathrm{l} & 5 \times \text { GoTaq buffer } \\ 0.1 \mu \mathrm{l} & \text { GoTaq polymerase }(1 \mathrm{U} / \mu \mathrm{l}) \\ 12.7 \mu \mathrm{l} & \mathrm{ddH}_{2} \mathrm{O}\end{array}$

PCR program

$\left[\begin{array}{ll}95^{\circ} \mathrm{C} & 3 \mathrm{~min} \\ 60^{\circ} \mathrm{C} & 30 \mathrm{sec} \\ 72^{\circ} \mathrm{C} & 45 \mathrm{sec} \\ 95^{\circ} \mathrm{C} & 30 \mathrm{sec}\end{array}\right] \times 30$


Tfeb genotyping PCR:

\section{Primers for Tfeb}

Sense primer:

5'-GCTTGGGACACCCACAGCTCC -3'

Antisense primer:

5'-GGACACCCCATAGAAAATCCG -3'

PCR master mix for GoTaq polymerase

$1.00 \mu \mathrm{l}$

$0.10 \mu \mathrm{l}$

$0.10 \mu \mathrm{l}$

$2.00 \mu \mathrm{l}$

$4.00 \mu \mathrm{l}$

$0.1 \mu \mathrm{l}$

$12.7 \mu \mathrm{l}$
DNA (100 pg-100 ng) sense primer (50 pM)

antisense primer (50 pM)

dNTP mix (2 mM)

5x GoTaq buffer

GoTaq polymerase $(1 \mathrm{U} / \mu \mathrm{l})$

$\mathrm{ddH}_{2} \mathrm{O}$
PCR program

$95^{\circ} \mathrm{C}$

$3 \mathrm{~min}$

$30 \mathrm{sec}$

$45 \mathrm{sec} \times 30$

$30 \mathrm{sec}$

1 min

$60^{\circ} \mathrm{C}$

$72^{\circ} \mathrm{C}$

$10 \mathrm{~min}$

$4^{\circ} \mathrm{C}$

pause

Octn3 genotyping PCR:

Primers for Octn3

Sense primer:

Antisense primer:

PCR master mix for GoTaq polymerase

PCR program

\begin{tabular}{|c|c|c|c|}
\hline $1.00 \mu \mathrm{l}$ & DNA (100 pg-100 ng) & $95^{\circ} \mathrm{C}$ & $3 \mathrm{~min}$ \\
\hline $0.10 \mu \mathrm{l}$ & sense primer (50 pM) & $60^{\circ} \mathrm{C}$ & $30 \mathrm{sec}$ \\
\hline $0.10 \mu \mathrm{l}$ & antisense primer (50 pM) & $72^{\circ} \mathrm{C}$ & $45 \mathrm{sec}$ \\
\hline $2.00 \mu \mathrm{l}$ & dNTP $\operatorname{mix}(2 \mathrm{mM})$ & $95^{\circ} \mathrm{C}$ & $30 \mathrm{sec}$ \\
\hline $4.00 \mu \mathrm{l}$ & $5 x$ GoTaq buffer & $60^{\circ} \mathrm{C}$ & $1 \mathrm{~min}$ \\
\hline $0.1 \mu \mathrm{l}$ & GoTaq polymerase $(1 \mathrm{U} / \mu \mathrm{l})$ & $72^{\circ} \mathrm{C}$ & $10 \mathrm{~min}$ \\
\hline $12.7 \mu \mathrm{l}$ & $\mathrm{dd} \mathrm{H}_{2} \mathrm{O}$ & $4^{\circ} \mathrm{C}$ & pause \\
\hline
\end{tabular}


ThyATPA genotyping PCR:

\section{Primers for ThyATPA}

Sense primer:

Antisense primer:
5'-TCTGAGTGGCAAAGGACCTTAGG-3'

5'- CGCTGAACTTGTGGCCGTTTACG-3'

PCR master mix for GoTaq polymerase

PCR program

\begin{tabular}{|c|c|c|c|c|}
\hline $1.00 \mu \mathrm{l}$ & DNA (100 pg-100 ng) & $95^{\circ} \mathrm{C}$ & $3 \mathrm{~min}$ & \\
\hline $0.10 \mu \mathrm{l}$ & sense primer (50 pM) & $60^{\circ} \mathrm{C}$ & $30 \mathrm{sec}$ & \\
\hline $0.10 \mu \mathrm{l}$ & antisense primer (50 pM) & $72^{\circ} \mathrm{C}$ & $30 \mathrm{sec}$ & x35 \\
\hline $2.00 \mu \mathrm{l}$ & $\mathrm{dNTP} \operatorname{mix}(2 \mathrm{mM})$ & $95^{\circ} \mathrm{C}$ & $30 \mathrm{sec}$ & \\
\hline $4.00 \mu \mathrm{l}$ & 5x GoTaq buffer & $60^{\circ} \mathrm{C}$ & $1 \mathrm{~min}$ & \\
\hline $0.1 \mu \mathrm{l}$ & GoTaq polymerase $(1 \mathrm{U} / \mu \mathrm{l})$ & $72^{\circ} \mathrm{C}$ & $10 \mathrm{~min}$ & \\
\hline $12.7 \mu \mathrm{l}$ & $\mathrm{dd}_{2} \mathrm{O}$ & $4^{\circ} \mathrm{C}$ & pause & \\
\hline
\end{tabular}




\subsection{Solution (aCSF) for optic nerve incubation and recording}

First, Stock solutions $A$ and $B$ were prepared as following for long term storage at $4^{\circ} \mathrm{C}$ :

\section{2x Stock A:}

$\begin{array}{lllll}\text { Salt } & \text { M.W. } & \text { [final mM] } & \text { at 2X (mM) } & \text { For 2 I (g) } \\ \mathrm{NaCl} & 58.44 & 126 & 252 & 29.45 \\ \mathrm{KCl} & 74.56 & 3 & 6 & 0.89\end{array}$

Phenol red

2x tockB:

$\begin{array}{lllll}\text { Salt } & \text { M.W. } & \text { [final } \mathbf{m M} \text { ] } & \text { at 2X (mM) } & \text { For 2 I (g) } \\ \text { NaH2PO4 } & 137.99 & 1.25 & 2.5 & 0.69 \\ \text { NaHCO3 } & 84.01 & 26 & 52 & 8.74 \\ \text { MgSO4 } & 246.48 & 2 & 4 & 1.97 \\ \text { Phenol red } & & 0.0001 & 0.0001 & \end{array}$

In the following step, using the prepared stocks, the aCSF containing substrates were made. Briefly, an equal volume of stock $A$ and $B$ were mixed and were bubbled with Carbogen (95\% O2, 5\% CO2) in a water bath with adjusted temperature at $37^{\circ} \mathrm{C}$. Glucose (or other substrates) was then added at a final concentration of $10 \mathrm{mM}$ to the solution and the bubbling was continued for another 1-2 h. At the end $\mathrm{CaCl} 2$ (final concentration of $2 \mathrm{mM}$ ) was added and after a few minutes of bubbling, the $\mathrm{pH}$ of the solution was measured to ensure that it is in the normal range $(\mathrm{pH}: 7.4-7.48)$. In order to maintain the osmolarity constant among solutions prepared for different conditions, $10 \mathrm{mM}$ of substrate was considered as standard and whenever concentrations lower than $10 \mathrm{mM}$ glucose (or other substrates: betahydroxybutyrate) was applied, the rest up to $10 \mathrm{mM}$ was replaced by sucrose (Merck Millipore, Darmstadt, Germany), since it cannot be metabolized by the cells. The prepared aCSF containing in mM: $126 \mathrm{NaCl}, 26 \mathrm{NaHCO}, 3 \mathrm{KCl}, 2 \mathrm{MgSO} 4,1.25 \mathrm{NaH} 2 \mathrm{PO} 4$ and $2 \mathrm{CaCl} 2,10$ substrates (or substrate + sucrose), $x x x$ phenol red was used for optic nerve incubation or electrophysiology recording. 


\subsection{Mouse optic nerve incubation and treatment}

After cervical dislocation, the mice were decapitated and the head skin was opened longitudinally, the eyeball was detached from the surrounding tissue, and the top of the skull was removed. After cutting the brain along the coronal plane in the middle, the optic nerve was cut at chiasmata and it was removed by pulling the eyeball gently. The prepared nerves attached to the eyeball were transferred into a six-well plate (Fig. 21) containing around $10 \mathrm{ml}$ of aCSF inside an incubator with adjusted temperature at $37^{\circ} \mathrm{C}$ (with/ without inhibitors + different metabolites) and another $90 \mathrm{ml}$ of ACSF was circulating during the incubation period. To provide oxygen to the nerve cells and maintain the $\mathrm{pH}$ of the solution constant during the incubation period (16 and $24 \mathrm{~h})$ the aCSF was continuously gassing with carbogen $(5 \% \mathrm{CO}$, $95 \%$ O2)

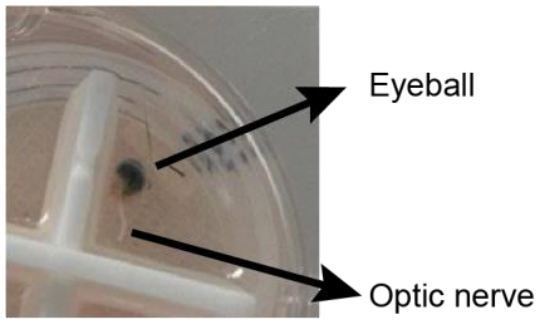

Figure 21. Prepared mouse optic nerve attached to the eyeball for incubation experiments. showing an example of acutely isolated mouse optic nerve (wild-type) for incubation experiments

In order to study the role FA on survival of the nerves during incubation, specific inhibitors for mitochondrial beta-oxidation, 4-Bromocrotonic acid $(25 \mu \mathrm{M})(\mathrm{TCI}, \geq 98 \%)$, peroxisomal beta-oxidation, Thioridzine (5 $\mu \mathrm{M}$ ) (Sigma Aldrich, $\geq 99 \%$ Germany) and mitochondrial beta-oxidation of long chain FA, Etomoxir ( $5 \mu \mathrm{M}$, Tocris) were added to aCSF right before starting the experiment. In order to block mitochondrial oxidative phosphorylation, $5 \mathrm{mM}$ sodium azide (Merck Millipore) was supplemented to aCSF acontaining 10- or 0-mM glucose. In this case, in order to compensate for sodium concentration, the used stock A solution for making aCSF contained $121 \mathrm{mM} \mathrm{NaCl}$.

In order to study the effect of hypoxia on survival of optic nerve cells during incubation, during the incubation time aCSF was constantly bubbling with nitrogen (95\% N2, 5\% CO2) 
instead of carbogen ( $95 \% \mathrm{O} 2,5 \% \mathrm{CO} 2)$ and to minimize the diffusion of oxygen into aCSF from the air, the wells were partially covered with pieces of parafilm (Merck).

\subsection{Cell death studies}

\subsubsection{PI staining and sectioning}

In order to detect the dead cells in the optic nerve, propidium iodide (PI) staining was performed. PI is an impermeable dye and cannot cross the plasma membrane of intact cells while it is able to enter the dead cells because of the change in membrane permeability. To stain the nerve cells with PI, the mouse optic nerves (MON) were treated with $11.97 \mu \mathrm{M}$ PI $1 \mathrm{~h}$ before the incubation times ended and nerves were washed for $10 \mathrm{~min}$ in $7 \mathrm{ml} \mathrm{ACSF}$. The stained nerves were fixed for $2 \mathrm{~h}$ in $4 \%$ paraformaldehyde (4\% PFA: NaH2PO4 (20mM), Na2HPO4 $(80 \mathrm{mM}), 4 \%$ paraformaldehyde) at $4^{\circ} \mathrm{C}$. In next step, the nerves were detached from eyeball and frozen blocks of nerves were made in Tissue-Tek ${ }^{\circledR}$ O.C.T ${ }^{\mathrm{TM}}$ compound (SAKURA, poland) for cryosectioning (cryostat, Leica). The frozen blocks of the optic nerve were left in cryostate for at least $60 \mathrm{~min}$ and $8 \mu \mathrm{m}$ thick longitudinal sections were collected on superfrost ${ }^{\circledast}$ Plus (Thermo Scientific) slides so that the sections on upper and lower part of the slides were related to the surface of the nerve and the sections in the middle were corresponding to the middle part of the nerve. The obtained sections were kept in the dark at $-20^{\circ} \mathrm{C}$ for other staining procedures.

\subsubsection{DAPI staining}

In order to count the total number of cells on each section the DAPI staining was performed. DAPI is an intercalating dye that stains the nucleus of the cells. The staining starts with 5 min washing the sections in PBS (In mM: $170 \mathrm{NaCl}, 3.4 \mathrm{KCl}, 4.0 \mathrm{Na} 2 \mathrm{HPO}$, $1.8 \mathrm{~K} 2 \mathrm{HPO}$, pH: 7.2) and followed by 10 min incubation with $200 \mu$ of DAPI solution in PBS (1:20000, stock: $1 \mathrm{mg} / \mathrm{ml}$ ). In the last step, the slides were washed for $5 \mathrm{~min}$ in PBS and subsequently dipped in distilled water before mounting with aquapolymountant (Polyscience Inc). During the staining procedure samples were protected from exposure to light until microscopy. 


\subsubsection{Imaging}

All the images were taken with an inverted epiflourescent microscope (Zeiss Axio Observer Z1) with $20 x$ objective with the same UV lamp intensity otherwise stated. The exposure time settings for the PI and DAPI channel were the same for all images for quantifying the dead cells. However, the exposure time for GFP or YFP channel for defining different cell types in the nerve was adjusted according to the strength of the signal in each transgenic line and it was kept constant for all the related images for each line. Two to three sections of each nerve were imaged and the resulting frames for each section were stitched using the microscope software to acquire the whole picture of each section for further quantifications.

\subsubsection{Cell death quantification}

In order to quantify the percent of dead cells for each cell type in the nerve, Fiji and Imaris softwares (version 8.1.2) were used. The obtained stitched images were opened in Fiji software to remove the margin, cut site and in case of crush, the crushed area of the nerve that contain dead cells unrelated to starvation conditions Subsequently, by using "plug in" option in Imaris, the opened images in Fijji were further quantified. After adjusting the threshold for each channel, the spots were created for each nucleus that has a diameter of around $3.22 \mu \mathrm{m}$. Since the dead cells have more PI dye intercalated in the DNA, sometimes the DAPI cannot be intercalated with enough efficiency. Therefore, the DAPI signal for the PI positive cells are weaker and the software does include them for quantification. To minimize the errors related to the signal, all the spots for each channel were double-checked and manually modified whenever necessary. In last step, the software calculated co-localizations between different channels and the data were exported as excel files for statistical analysis.

\subsection{Proteomics and expression analysis}

\subsubsection{Protein extraction from optic nerve:}

In order to minimize the variability between the samples and genetic background, one nerve of each mouse was incubated under starvation condition and the other one under normal 
condition and the nerves from each two mice were pooled together for protein extraction. The nerves were collected after $24 \mathrm{~h}$ incubation in aCSF $+10 \mathrm{mM}$ or $1 \mathrm{mM}$ (starvation) glucose, transferred into microtubes, and kept at $-80^{\circ} \mathrm{Cfor}$ further analysis. The collected nerves were homogenized in $70 \mu \mathrm{l}$ of RIPA buffer (Tris $\mathrm{Hcl}(50 \mathrm{mM}), \mathrm{Na}$-deoxycholate $(0.5 \%), \mathrm{NaCl}(150$ $\mathrm{mM})$, SDS (0.1\%), Triton X100 (1\%), EDTA (1 mM) and complete protease inhibitor cocktail (Roche, Mannheim, Germany)) using ceramic beads in a Precellys homogenizer. The homogenization was performed three times and each time was composed of $3 \times 10 \mathrm{sec}$ at $6500 \mathrm{rpm}$. The samples were kept on ice between each round of homogenization. The resulted lysates were transferred into $1.5 \mathrm{ml}$ Eppendorf tubes and centrifugation was performed for five minutes at $13000 \mathrm{rpm}$ at $4{ }^{\circ} \mathrm{C}$. The obtained supernatant was gently removed and transferred into new tubes for further analysis and the pellets were kept at $-80^{\circ} \mathrm{C}$.

\subsubsection{Protein concentration measurement}

Protein concentration in the collected supernatant was measured using the Lowry assay. The assay had two steps: in the first step, the copper ions react with peptide bonds in the protein under alkaline condition and in the second step, the Folin reagent is reduced into a colorful product by copper treated proteins (Lowry et al., 1951). The Bio Rad DC Protein Assay reagents were purchased (Bio Rad) and protein measurement was performed according to the manufacturer protocol. Briefly, $20 \mu \mathrm{l}$ of reagent S was added to each $\mathrm{ml}$ of reagent A. $25 \mu \mathrm{l}$ of reagent $A(+S)$ was added for every sample to a 96 well plate (flat bottom). Then $5 \mu$ l of standard solution or sample were added and after adding $200 \mu$ l of reagent B to each well the plate was incubated at room temperature for $15 \mathrm{~min}$. After reading the absorbance of the samples at 736 $\mathrm{nm}$ (Eon microplate spectrophotometer, Biotek instruments), the protein concentration was calculated based on the standard curve.

\subsubsection{SDS-PAGE}

In order to evaluate the integrity of the samples for proteomics, proteins were separated by SDS-PAGE and the gel was subjected to silver staining. The samples were added to loading 
buffer containing $5 \%$ beta-Mercaptoethanol and after 10 min incubation at $40^{\circ} \mathrm{C}$ with shaking (550 rpm) they were ready for loading on the gel. The desired amount of protein was loaded on a $12 \%$ polyacrylamide gel next to a pre-stained protein marker (ThermoScientific) and separation of proteins was performed in a runninsg tank (Biorad) filled with running buffer (Laemmli-buffer) by applying appropriate current (voltage: 120; current: $300 \mathrm{~mA}$ ) for around $60 \mathrm{~min}$. At the end of electrophoresis, the obtained gel was used for silver staining or for immunoblotting.

\subsubsection{Silver staining}

In order to evaluate the integrity of extracted proteins from incubated optic nerves for proteomics studies, $0.5 \mu \mathrm{g}$ of the protein was separated on $12 \%$ gel by doing SDS-PAGE and was subjected to silver staining (Sørensen et al., 2002). In fixation step, the gel was incubated in gel fixing solution (containing 40\% (v/v) Ethanol and 10\% (v/v) Acetic acid in dd $\mathrm{H} 2 \mathrm{O}$ ) for at least 60 min (or overnight). After two times incubating the gel in 30\% (v/v) Ethanol for $20 \mathrm{~min}$, it was washed with dd $\mathrm{H} 2 \mathrm{O}$ for another 20 min. In sensitization step, the gel was treated with sodium thiosulfate $(797.7 \mu \mathrm{M}$, Merck) for $1 \mathrm{~min}$ and after washing it with ddH2O $(3 \times 20 \mathrm{sec})$ it was used for impregnation by incubating it in silver nitrate $(11.77 \mathrm{mM})$ for $20 \mathrm{~min}$. After short steps of washing in dd $\mathrm{H} 2 \mathrm{O}(3 \times 20 \mathrm{sec})$ the color was developed by adding sodium carbonate $(283 \mu \mathrm{M})$ until a sufficient and detectable signal appears. The reaction was then stopped by washing the gel in $5 \%(\mathrm{v} / \mathrm{v})$ acetic acid $(2 \times 10 \mathrm{~min})$. The stained gel was washed in water and after imaging for long storage was kept in dd $\mathrm{H} 2 \mathrm{O}$ at $4{ }^{\circ} \mathrm{C}$.

\subsubsection{Brain myelin preparation}

Glut1 $1^{f / f l} \times P / p C r e T 2^{+/-}$-mice and their corresponding Glut1 ${ }^{f / f f}$-control mice (without Plp $\left(\mathrm{CreT}^{+/}\right)$were sacrificed at four months post induction of recombination by tamoxifen injection. A light weighted membrane fraction enriched in myelin was obtained from frozen half brains by sucrose density gradient centrifugation essentially as described (Erwig et al., 2019). Briefly, after homogenizing the brains in $0.32 \mathrm{M}$ sucrose solution containing protease 
(complete, Roche), a first fraction enriched in myelin was obtained by density gradient centrifugation on a $0.85 \mathrm{M}$ sucrose cushion. After washing and two consecutive osmotic shocks, the final brain myelin fraction was purified by sucrose gradient centrifugation as before. The myelin fraction was washed and suspended in TBS buffer $(137 \mathrm{mM} \mathrm{NaCl}, 20 \mathrm{mM}$ Tris/HCl, $\mathrm{pH}$ $7.4,4^{\circ} \mathrm{C}$ ) supplemented with protease inhibitors (complete, Roche). Protein concentration was determined using the DC Protein assay (Biorad) according to the manufacturer's instructions as mentioned above.

\subsubsection{Western blotting}

For immunoblotting, the proteins were separated by doing SDS-PAGE as described above. After activating polyvinylidene difluoride membranes (Immobilon-FL PVDF, IPFL00010, Merck-Millipore) by incubation in methanol for 1 min, a sandwich of the gel, PVDF membrane and Whatman papers was prepared and proteins were blotted onto the PVDF membrane in a wet blotting chamber (Biorad) filled with transfer buffer $(10 \%(\mathrm{v} / \mathrm{v})$ methanol, $48 \mathrm{mM}$ tris-base, $39 \mathrm{mM}$ glycine and $0.003 \%(\mathrm{w} / \mathrm{v})$ SDS). For quantification of total protein, blots were stained by fast green (5 mg/L fast green, Sigma, in $6.7 \%[\mathrm{v} / \mathrm{v}]$ acetic acid, $30 \%[\mathrm{v} / \mathrm{v}]$ methanol) immediately after transfer for 5 min, washed twice for $30 \mathrm{sec}$ in $6.7 \%$ [v/v] acetic acid, $30 \%$ $[\mathrm{v} / \mathrm{v}]$ methanol and imaged using the $700 \mathrm{~nm}$ channel of a near-infrared scanned (Odyssey, Licor). Consecutively, blots were destained in $50 \%[\mathrm{v} / \mathrm{v}]$ ethanol in TBS $(150 \mathrm{mM} \mathrm{NaCl}, 10 \mathrm{mM}$ Tris/ $\mathrm{HCl}$, pH 7.4) Blocking was performed with $5 \%$ [w/v] skim milk in TBST (150mM NaCl, $10 \mathrm{mM}$ Tris/ $\mathrm{HCl}, \mathrm{pH} 7.4,0.5 \%[\mathrm{v} / \mathrm{v}]$ Tween 20) for $60 \mathrm{~min}$ at $\mathrm{RT}$ and subsequently the membrane was incubated with primary antibodies diluted in the same solution ((GLUT1 (1:1000, Berghoff et al., 2017), GLUT2 (1:1000, ab54460, abcam), GLUT3 (1:1000, ab191071, abcam), GLUT4 (1:1000, 07-1404, Millipore), MCT1 (1:1000, Stumpf et al., 2019), carbonic anhydrase 2 (CA2, 1:1000, Ghandour et al., 1980) and $\alpha$-tubulin (TUBA, 1:1000, T 5168, Sigma)) for $48 \mathrm{~h}$ at $4^{\circ} \mathrm{C}$. In the next step, after washing with TBST ( $3 \times 15$ min on the shaker), blots were incubated with nearinfrared fluorophore coupled to secondary antibodies diluted in $5 \%[\mathrm{w} / \mathrm{v}]$ skim milk in TBST (1:10000, Mouse IgG (H\&L) Antibody Dylight ${ }^{\mathrm{TM}} 680$ Conjugated, 610-144-002; Rabbit IgG (H\&L) Antibody DyLight ${ }^{\mathrm{TM}} 800$ Conjugated, 611-145-002; Rockland), washed with TBST, finally TBS 
and detected using a near-infrared fluorescence scanner (Odyssey, Licor). For quantification of GLUT1, GLUT3 and MCT1, images obtained from the near-infrared fluorescence imager were analyzed using the Image Studio software (Licor). Signal intensities were normalized to their corresponding total protein load as quantified by fast green staining. Normalized signal

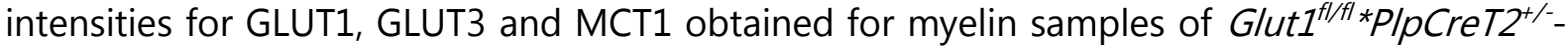
mice were normalized to the mean of the respective signal intensities of myelin samples from Glut1 $^{f / f f}$-control mice.

\subsection{Studying myelin thickness}

\subsubsection{Electron microscopy}

In order to study the ultrastructure of myelin, mouse optic nerves were subjected to electron microscopy. The animals were sacrificed by cervical dislocation and the freshly prepared optic nerves (or incubated nerves) were fixed in Karlsson and Schultz fixative (4 \% formaldehyde, $2.5 \%$ glutaraldehyde, $0.5 \% \mathrm{NaCl}$, in phosphate buffer $\mathrm{pH} 7.4$ ) overnight at $4{ }^{\circ} \mathrm{C}$. The following program was used for embedding the samples using an automated device (EMTP, Leica). The fixed samples were washed in buffer and osmified. After washing the osmified samples with buffer they were subjected to dehydration steps using acetone, which was replaced by Epon. Epon was prepared by mixing $171.3 \mathrm{~g}$ Glycidether 100 (Serva, 21045), $115 \mathrm{~g}$ Dodecenyl succinic anhydride (Serva, 20755) and $89 \mathrm{~g}$ of Methyl nadic anhydride (Serva, 29452) using a magnetic stirrer for $10 \mathrm{~min}$. After adding $6.5 \mathrm{ml}$ of DMP-30 (Serva, 36975) and stirring for another $20 \mathrm{~min}$, the solution was ready to use. The treated samples (with above mentioned program) were placed in prepared Epon and the blocks from the samples was formed by incubation at $60^{\circ} \mathrm{C}$, overnight. Sections with $50-60 \mathrm{~nm}$ thickness were prepared by cutting the Epon embedded tissues using a Leica Ultracut S ultramicrotome (Leica, Vienna, Austria) equipped with a diamond blade (Diatome Hist $45^{\circ}$ and Ultra $45^{\circ} \mathrm{C}$ ). The sections were placed on a grid and imaged using a LEO EM 912AB electron microscope (Zeiss, Oberkochen, Germany) equipped with an on-axis 2048x2048-CCD-camera (TRS, Moorenweis, Germany). 


\section{Embedding program}

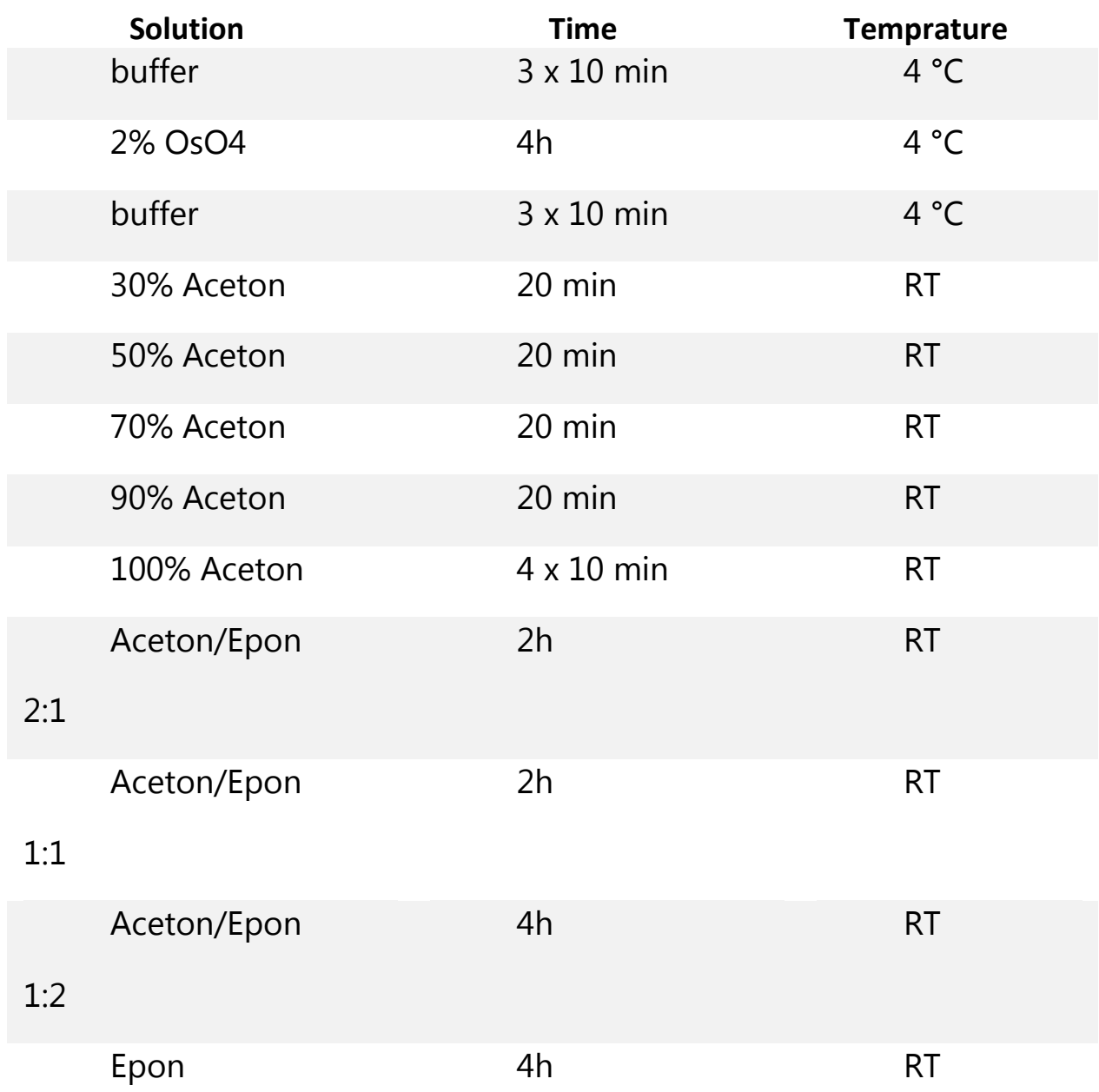

Embedding, sectioning and transmission electron microscopy imaging were kindly performed by Dr. Weibke Möbius, Torben Ruhwedel, Boguslawa Sadowski, - Neurogenetics department, Max-Planck Institute for Experimental Medicine.

\subsubsection{G-ratio analysis}

The obtained EM images from optic nerve of GLUT1 ciKO mice were opened with the Fijji software. In order to unbiasedly select the axons for quantification, a grid composed of cubes with $4 \mu \mathrm{m}^{2}$ area was created on each image and the axons that were hit by the cross were filtered with the following criteria for quantification: 1) it should be in cross section, 2) it has enough quality for quantification, 3 ) it is not too much stretched in shape (judged by eye). For each axon, three circles were made around it corresponding to the inner tongue and the outer layer of myelin. Whenever the structure of the myelin around one axon was not preserved 
evenly, the thickness of myelin in adjacent preserved area was used as a reference and circle was corrected accordingly. The obtained area of each circle was converted into diameter and the g-ratio was calculated based on diameter values (at least 100 axons/nerve was quantified). Quantification for incubated nerve for $24 \mathrm{~h}$ was performed slightly different from GLUt1 ciKO mice and the quantifiable axons were selected for quantification instead of using grid.

\subsection{Mouse optic nerve electrophysiology and live imaging}

In order to monitor both neuronal function and ATP level in axons, electrophysiology of optic nerve using suction electrodes was combined with live imaging. The optic nerve from THYATPA mouse line (expressing a FRET sensor for ATP in their axons) was clamped using suction electrodes filled with aCSF containing $10 \mathrm{mM}$ glucose as illustrated in Fig. 22.

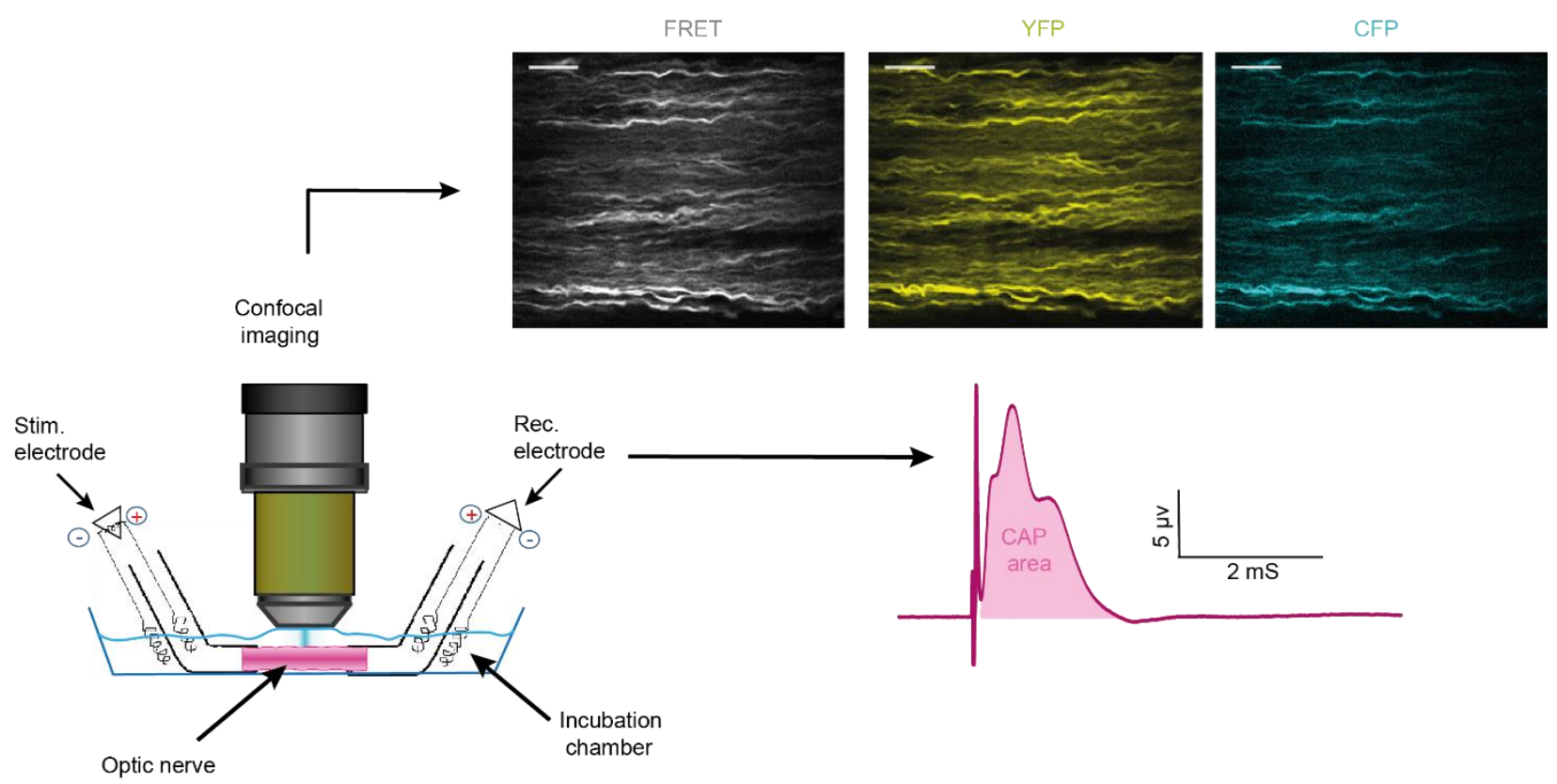

Figure 22. Showing a scheme of combined electrophysiology and confocal imaging for monitoring neuronal function and ATP level in optic nerve from mouse line expressing ATP sensor in their axons.

The placed optic nerve (in pink) in a recording chamber continuously perfused with aCSF. The electrode on the left side (stim. Electrode) of the chamber inject a current into the nerve and on right side the signal is recorded using recording electrode (Rec. electrode). The obtained signal (left side, down) has three peaks related to axons with different speed of signal propagation. The area under the signal is representing the function of all axons in nerve and is called compound action potential area (CAP area). While the nerve is receiving electrical stimulation, the objective on top of the nerve acquiring images every $\approx 30 \mathrm{sec}$ at three different channels (top, from left to right: FRET, YFP, CFP; frame size: $114.5 \mu \mathrm{m} \times 133.5 \mu \mathrm{m}$; scale bar: $20 \mu \mathrm{m}$ ). 


\subsubsection{CAP Recording}

All the mice used for optic nerve electrophysiology were at the age of 8-9 week unless otherwise stated. After cervical dislocation and decapitation, the optic nerve was detached from the eyeball and after removing the top of the skull the brain was pulled out carefully and the nerve was cut at chiasmata. The prepared nerves were transferred into a recording chamber (Harvard Apparatus, Holliston, MA) with continuous perfusion with aCSF (containing in mM: $126 \mathrm{NaCl}, 26 \mathrm{NaHCO}, 3 \mathrm{KCl}, 2 \mathrm{MgSO} 4,1.25 \mathrm{NaH} 2 \mathrm{PO} 4$ and $2 \mathrm{CaCl} 2,10$ substrate). To maintain the temperature at $37{ }^{\circ} \mathrm{C}$ during the experiments, the chamber was equipped with a temperature controller (model TC-10, NPI electronic) connected to a temperature sensor (TS100-S; NPI electronic) inserted in the chamber in direct contact with aCSF. In order to provide oxygen to the nerve cells during recording and maintain the $\mathrm{pH}$ of aCSF constant, the aCSF was continuously bubbling with carbogen (95\% O2, 5\% CO2). The optimal stimulation and recording condition were ensured by back filling the suction electrodes (custom-made) with aCSF containing $10 \mathrm{mM}$ glucose in all experiments. To achieve supermaximal stimulation, the stimulating electrode connected to the battery (Stimulus Isolator 385; WPI, Berlin, Germany) was injecting a current of $0.75 \mathrm{~mA}$ magnitude into the proximal end of the optic nerve with each stimulation. The produced signal detected at the distal end of the nerve using recording electrode connected to the headstage (Heka Electronik, Germany), amplified 200 or 500 times and after filtering at $30 \mathrm{kHz}$ (low noise amplifier, Stanford, USA) was acquired at $100 \mathrm{kHz}$ with an EPC9 amplifier (Heka Elektronik, Lambrecht/Pfalz, Germany). Acquired signal was amplified further using Ext 10-2F amplifier (NPI electronic) and low-noise voltage preamplifier SR560 (Stanford Research System) 10 times and 20-50 fold respectively (Stys et al., 1991; Saab et al., 2016; Trevisiol et al., 2017).

For making suction electrodes, glass capillaries (1.5 mm, \#1B150-6, World Precision Instruments) were heated at one end on the flame and manually were bended to an angle that fits the recording chamber. Silver wires with $0.25 \mathrm{~mm}$ thickness (World Precision Instruments) were coated with chloride and at one end they were attached to the gold pin. In the stimulating electrode, one of the wires was inserted into the glass capillary and the other one was wrapped around it and the wires were connected to positive and negative poles of the battery. In the 
recording electrode composed of two capillaries, the wires were placed inside capillaries and were connected to the headstage. The glass parts and wires were sealed together using the heat-shrinking tubes and were attached to two arms equipped with two micromanipulators (Luigs \& Neumann) for adjusting the positions of the electrodes on the recording chamber. All the recordings were done after nerve equilibration for $2 \mathrm{~h}$ in aCSF containing $10 \mathrm{mM}$ glucose as baseline otherwise stated. During baseline recording the nerves were evoked every $30 \mathrm{sec}$ and for higher frequencies a burst-stimulation was applied, and the signal was recorded every $~ 30$ sec.

\subsubsection{Imaging}

An upright confocal laser scanning microscope (Zeiss LSM 510 META/NLO, Zeiss, Oberkochen, Germany) equipped with an Argon laser and a 63x objective (Zeiss 63x IRAchroplan $0.9 \mathrm{~W}$ ) was used for live imaging of the optic nerve. After putting the nerve into the suction electrodes, the objective gently immersed into the aCSF superfusing the optic nerve and images were acquired with a time resolution of around $30 \mathrm{sec}$. The imaging settings were adjusted as reported previously (Trevisiol et al., 2017). A frame size of $\approx 114.5 \mu \mathrm{m} \times 133.5 \mu \mathrm{m}$ (384 x 448 pixels) was scanned for CFP (excitation 458 nm; emission 470-500 nm), FRET (Ex 458 nm; Em long pass $530 \mathrm{~nm}$ ) and YFP (Ex $514 \mathrm{~nm}$; Em long pass $530 \mathrm{~nm}$ ).

\subsubsection{CAP analysis}

Optic nerve function can be measured quantitatively by calculating the area under the signal that is representative of function of all nerve axons, referred as compound action potential (CAP) area. Optic nerves are a heterogeneous structure composed of a variety of axons with different rate of signal propagation. The obtained signal from the optic nerve includes three peaks that are representative of different axons with different rate of signal speed (Stys et al., 1991; Saab et al., 2016; Trevisiol et al., 2017). The signal related to the fastest group of axons is appeared in first peak and the second and third peaks are related to axons with medium and slow rates of signal propagation. To select the time window for calculating the CAP area, the start of the first peak and end of third peak of the recorded signal at the last 
few minutes of the baseline, when the signal is very stable, were defined. This time window was then applied to all the recorded traces for calculating the CAP area. After normalizing the data to the average of CAP area values obtained from the last $30 \mathrm{~min}$ of the baseline, the results from several nerves were pooled, averaged and plotted against time. In order to present the overall function of the nerve during a desired time window, the area under the CAP area curve was calculated and presented as area under the curve for each nerve after normalizing the values to the mean value of control nerves, bar graphs were plotted for them.

\subsubsection{ATP quantification}

The relative level of ATP was calculated as previously reported (Trevisiol et al., 2017). The images were opened in Fiji and the area of the nerve that was stable during the imaging were selected for measuring the mean intensity for three different channels: FRET, CFP and YFP. Then the ratio of FRET /CFP as relative ATP amount was calculated and normalized to zero and one by using the values obtained during the mitochondrial ATP blockade ( $5 \mathrm{mM} \mathrm{Azide)}$ and baseline (10 mM glucose) steps respectively.

\subsection{Data presentation}

All data are presented as mean \pm SEM. for data regarding cell death measurements in the optic nerve, the data of at least two technical replicates were combined and the mean was considered as one data point. Therefore, $\mathrm{N}$-number indicates biological replicates. The number of optic nerves analyzed for each condition is given as $\mathrm{n}$. As in all experiments only one nerve of each mouse was included for each condition, the number of nerves is equal to the number of animals analyzed for each condition. However, there is an exception about samples for proteomics measurements that two nerves were pooled together. To see if there is a significant difference between different conditions, the data were statistically evaluated using Welch's ttest and normal distribution was assumed $\left({ }^{*} p<0.05 ;{ }^{* *} p<0.01 ;{ }^{* *} p<0.001\right)$. Apart from the used Microsoft excel for data analysis, Microsoft Office and Adobe illustrator softwares were used for preparing figures and writing this thesis. 


\subsection{MATLAB Script for CAP analysis}

The recorded signal in Patchmaster are saved as .dat files and after being exported as .mat files they were processed by the following script in MATLAB (written by Dr. Andrea Trevisiol).

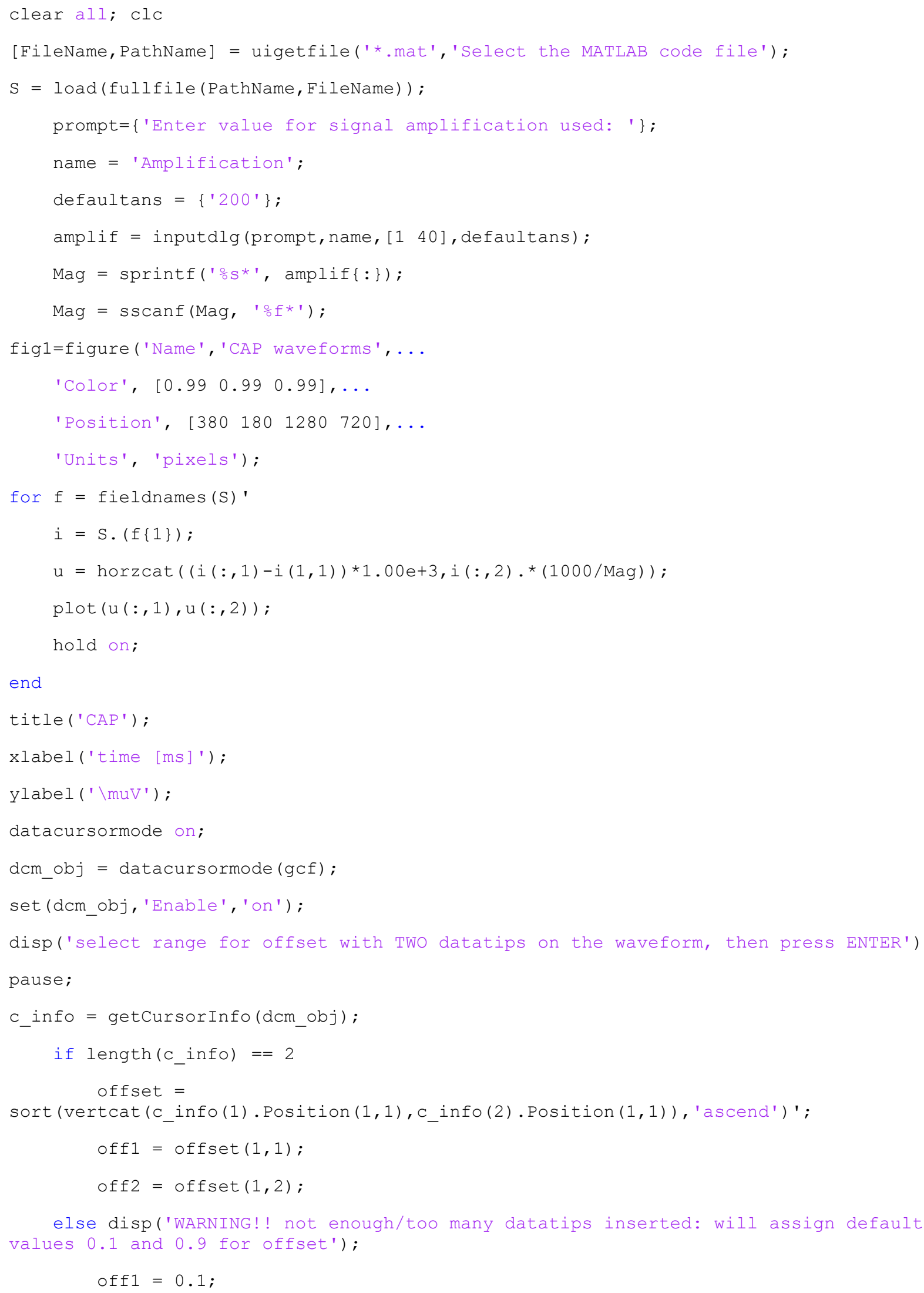


off2 $=0.9$;

pause

end

datacursormode off;

hold off

for $\mathrm{f}=$ fieldnames $(\mathrm{S})^{\prime}$

$i=S \cdot(f\{1\})$;

$u=\operatorname{horzcat}((i(:, 1)-i(1,1)) * 1.00 e+3, i(:, 2) . *(1000 /$ Mag $))$;

$r=\operatorname{horzcat}(u(:, 1), u(:, 2)-$ mean $(u(m i n(f i n d(u(:, 1)\rangle$

offl\&u $(:, 1)<$ off2) ) : $\max ($ find $(u(:, 1)>\operatorname{off} 1$ \&u $(:, 1)<$ off2) ), 2) )) ;

$r(:, 2)=$ sgolayfilt $(r(:, 2), 4,23)$;

$\operatorname{plot}(r(:, 1), r(:, 2))$;

hold on;

end

datacursormode on;

dcm_obj = datacursormode(gcf);

set (dcm_obj, 'Enable', 'on') ;

disp('select range for CAP Area Analysis, then press ENTER')

pause;

c_info2 = getCursorInfo(dcm_obj);

range $=$ sort(vertcat(c_info2(1).Position(1,1), c_info2(2).Position(1,1)), 'ascend') ';

left $=$ range $(1,1)$

right $=$ range $(1,2)$;

if c_info2(1).Position $(1,1)>c_{-}$info2(2). Position $(1,1)$;

xcap = c_info2(1). Target.XData;

ycap $=$ c_info2(1). Target.YData;

else

xcap $=$ c_info2(2). Target.xData;

ycap $=c_{\text {_info2 }(2)}$. Target. YData

end

hold on

xauc $=[$ xcap $($ xcap $>=$ left $\&$ xcap $\langle$ right $) ;$ xcap $($ xcap $>=$ left\&xcap $\langle$ right $) ;$

xcap (xcap>left \&xcap<=right); xcap (xcap>left\&xcap<=right) ] ;

yauc $=[\operatorname{zeros}(1$, length $($ xauc $)) ; \operatorname{ycap}($ xcap $>=$ left\&xcap<right $) ;$

ycap (xcap>left\&xcap<=right); $\operatorname{zeros}(1$, length (xauc)) ] ;

p = patch (xauc, yauc, [.99 .2 .7], 'FaceAlpha', '.2','EdgeColor', 'none');

title ('CAP');

xlabel ('time [ms]');

ylabel ('\muV');

$f=$ fieldnames $(S)$ '; 


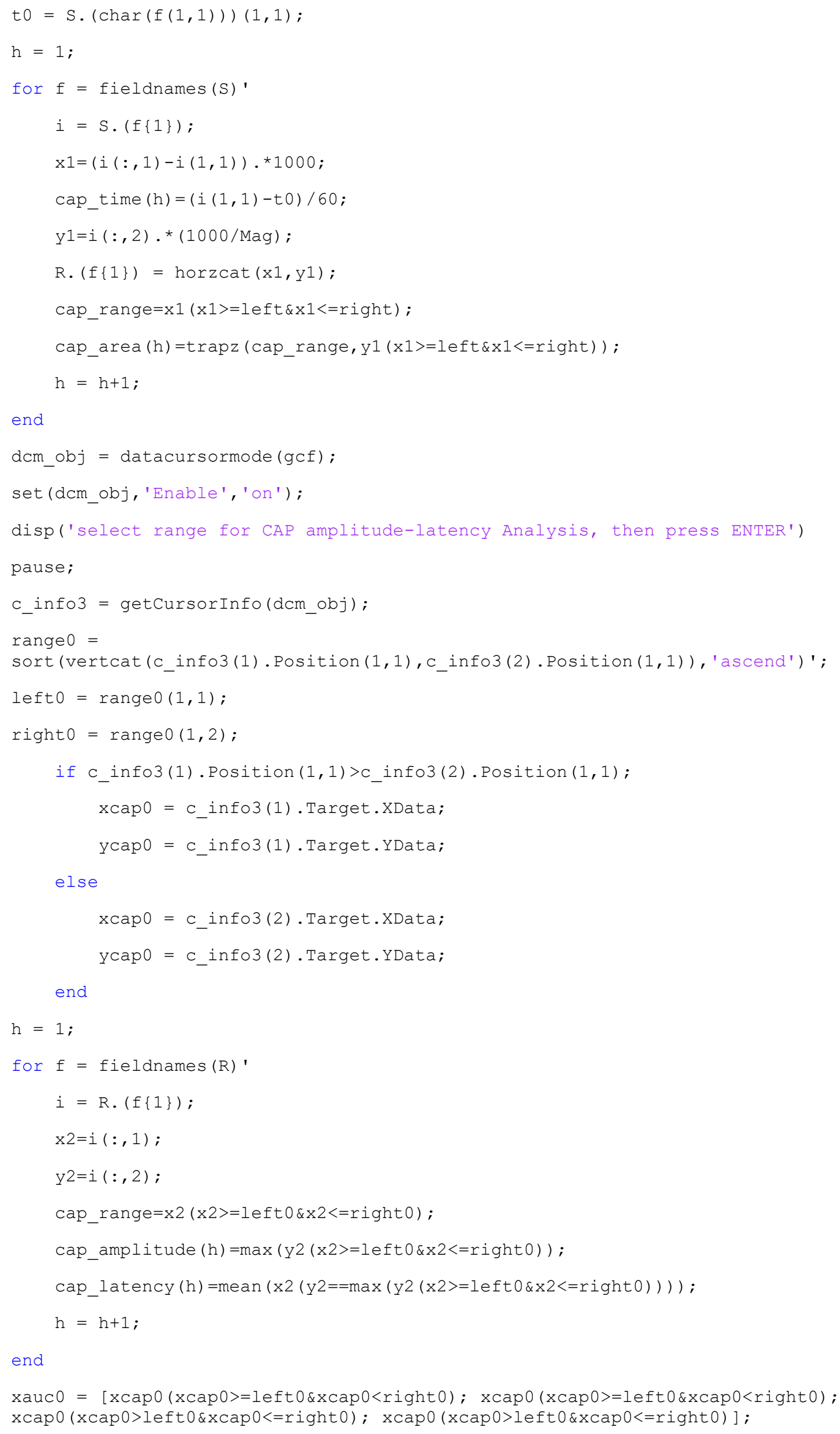




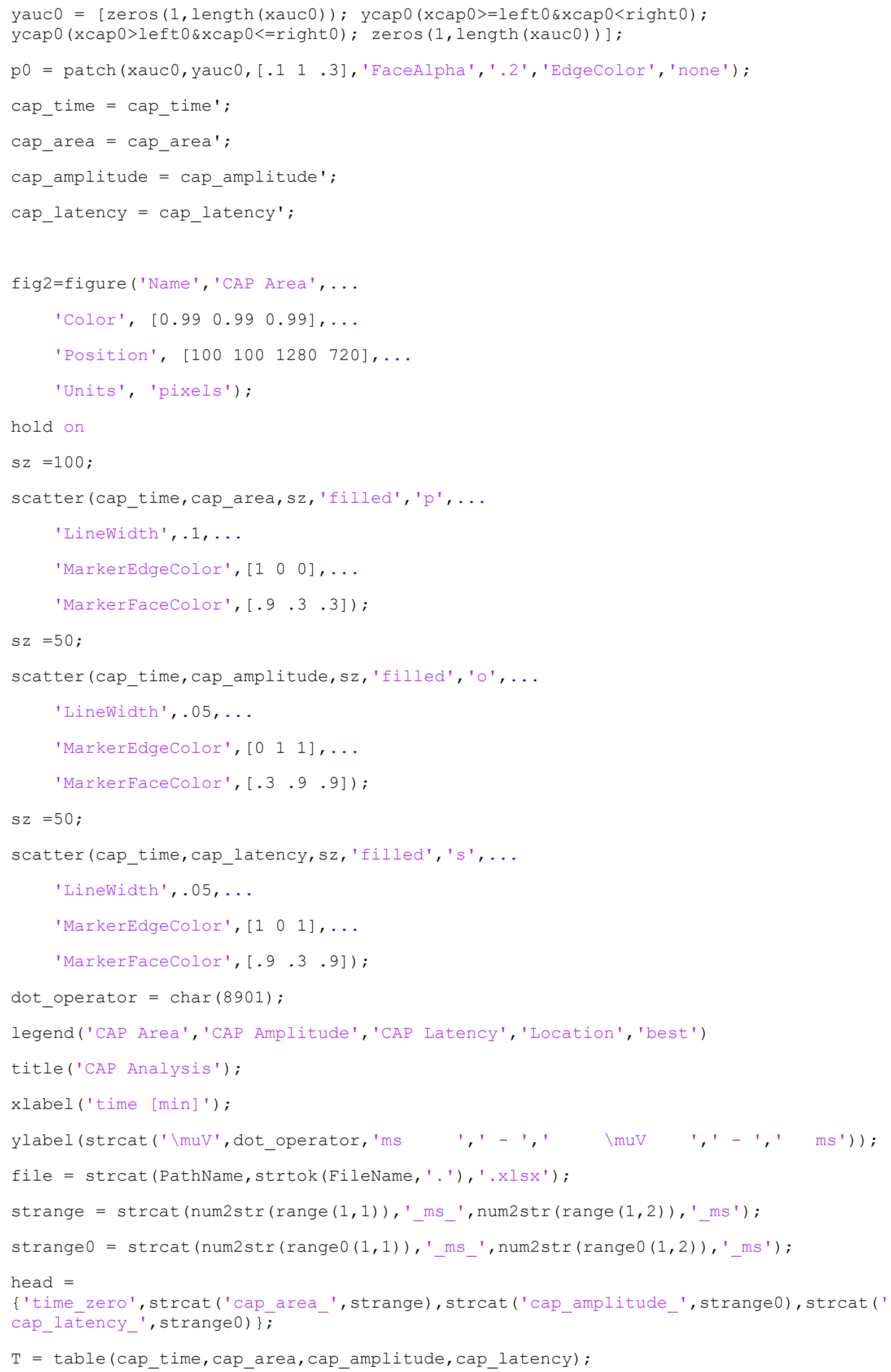


writetable (T,file, 'Sheet', 1):

xlswrite(file, head, 1, 'A1:D1'); 


\section{References}

Abdel-aleem S, Li X, Anstadt M, Perez-Tamayo R, Lowe J. 1994. Regulation of Glucose Utilization During the Inhibition of Fatty Acid Oxidation in Rat Myocytes. Horm Metab Res 26:88-91. doi:10.1055/s-2007-1000779

Abumrad NA, El-Maghrabi MR, Amri EZ, Lopez E, Grimaldi PA. 1993. Cloning of a rat adipocyte membrane protein implicated in binding or transport of long-chain fatty acids that is induced during preadipocyte differentiation. Homology with human CD36. J Biol Chem 268:17665-17668.

Arancibia-Cárcamo IL, Ford MC, Cossell L, Ishida K, Tohyama K, Attwell D. 2017. Node of Ranvier length as a potential regulator of myelinated axon conduction speed. Elife 6:1-15. doi:10.7554/eLife.23329

Atshaves BP, Martin GG, Hostetler HA, McIntosh AL, Kier AB, Schroeder F. 2010. Liver fatty acidbinding protein and obesity. J Nutr Biochem 21:1015-1032. doi:10.1016/j.jnutbio.2010.01.005

Auestad N, Korsak RA, Morrow JW, Edmond J. 1991. Fatty Acid Oxidation and Ketogenesis by Astrocytes in Primary Culture. J Neurochem 56:1376-1386. doi:10.1111/j.14714159.1991.tb11435.x

Baes M, Van Veldhoven PP. 2012. Mouse models for peroxisome biogenesis defects and $\beta$ oxidation enzyme deficiencies. Biochim Biophys Acta - Mol Basis Dis 1822:1489-1500. doi:10.1016/j.bbadis.2012.03.003

Baltan S. 2014. Can lactate serve as an energy substrate for axons in good times and in bad, in sickness and in health? Metab Brain Dis 30:25-30. doi:10.1007/s11011-014-9595-3

Berghoff SA, Gerndt N, Winchenbach J, Stumpf SK, Hosang L, Odoardi F, Ruhwedel T, Böhler C, Barrette B, Stassart R, Liebetanz D, Dibaj P, Möbius W, Edgar JM, Saher G. 2017. Dietary cholesterol promotes repair of demyelinated lesions in the adult brain. Nat Commun 8:14241. doi:10.1038/ncomms14241

Bittar PG, Charnay Y, Pellerin L, Bouras C, Magistretti PJ. 1996. Selective Distribution of Lactate Dehydrogenase Isoenzymes in Neurons and Astrocytes of Human Brain. J Cereb Blood Flow Metab 16:1079-1089. doi:10.1097/00004647-199611000-00001

Black JA, Waxman SG. 1988. The perinodal astrocyte. Glia 1:169-183. doi:10.1002/glia.440010302 Bottelbergs A, Verheijden S, Hulshagen L, Gutmann DH, Goebbels S, Nave K-A, Kassmann C, Baes M. 2010. Axonal integrity in the absence of functional peroxisomes from projection neurons and astrocytes. Glia 58:1532-1543. doi:10.1002/glia.21027 
Bröer S, Rahman B, Pellegri G, Pellerin L, Martin J-L, Verleysdonk S, Hamprecht B, Magistretti PJ. 1997. Comparison of Lactate Transport in Astroglial Cells and Monocarboxylate Transporter 1 (MCT 1) Expressing Xenopus laevis Oocytes. J Biol Chem 272:30096-30102. doi:10.1074/jbc.272.48.30096

Brown AM, Tekkök SB, Ransom BR. 2004. Energy transfer from astrocytes to axons: The role of CNS glycogen. Neurochem Int 45:529-536. doi:10.1016/j.neuint.2003.11.005

Brown AM, Tekkök SB, Ransom BR. 2003. Glycogen Regulation and Functional Role in Mouse White Matter. J Physio/549:501-512. doi:10.1113/jphysiol.2003.042416

Brown AM, Wender R, Ransom BR. 2001. Metabolic substrates other than glucose support axon function in central white matter. J Neurosci Res 66:839-843. doi:10.1002/jnr.10081

Cahill GF. 2006. Fuel Metabolism in Starvation. Annu Rev Nutr 26:1-22. doi:10.1146/annurev.nutr.26.061505.111258

Cahoy JD, Emery B, Kaushal A, Foo LC, Zamanian JL, Christopherson KS, Xing Y, Lubischer JL, Krieg PA, Krupenko SA, Thompson WJ, Barres BA. 2008. A Transcriptome Database for Astrocytes, Neurons, and Oligodendrocytes: A New Resource for Understanding Brain Development and Function. J Neurosci 28:264-278. doi:10.1523/JNEUROSCI.4178-07.2008

Chegary M, Brinke H te, Ruiter JPN, Wijburg FA, Stoll MSK, Minkler PE, van Weeghel M, Schulz H, Hoppel CL, Wanders RJA, Houten SM. 2009. Mitochondrial long chain fatty acid $\beta$-oxidation in man and mouse. Biochim Biophys Acta - Mol Cell Biol Lipids 1791:806-815. doi:10.1016/j.bbalip.2009.05.006

Chrast R, Saher G, Nave K-A, Verheijen MHG. 2011. Lipid metabolism in myelinating glial cells: lessons from human inherited disorders and mouse models. J Lipid Res 52:419-434. doi:10.1194/jlr.R009761

Cocco T, Di M, Papa P, Lorusso M. 1999. Arachidonic acid interaction with the mitochondrial electron transport chain promotes reactive oxygen species generation. Free Radic Biol Med 27:51-59. doi:10.1016/S0891-5849(99)00034-9

Crowley LC, Scott AP, Marfell BJ, Boughaba JA, Chojnowski G, Waterhouse NJ. 2016. Measuring Cell Death by Propidium Iodide Uptake and Flow Cytometry. Cold Spring Harb Protoc 2016:pdb.prot087163. doi:10.1101/pdb.prot087163

De Duve C. 1996. The peroxisome in retrospect. Ann N Y Acad Sci 804:1-10. doi:10.1111/j.17496632.1996.tb18603.x

de Leon MJ, Ferris SH, George AE, Christman DR, Fowler JS, Gentes C, Reisberg B, Gee B, Emmerich M, Yonekura Y, Brodie J, Kricheff II, Wolf AP. 1983. Positron emission tomographic studies of aging and Alzheimer disease. AJNR Am J Neuroradio/ 4:568-71. 
Ding F, Yao J, Rettberg JR, Chen S, Brinton RD. 2013. Early Decline in Glucose Transport and Metabolism Precedes Shift to Ketogenic System in Female Aging and Alzheimer's Mouse Brain: Implication for Bioenergetic Intervention. PLoS One 8:e79977. doi:10.1371/journal.pone.0079977

Douglas Fields R. 2015. A new mechanism of nervous system plasticity: activity-dependent myelination. Nat Rev Neurosci 16:756-767. doi:10.1007/s11065-015-9294-9.Functional Ebert D, Haller RG, Walton ME. 2003. Energy Contribution of Octanoate to Intact Rat Brain Metabolism Measured by 13 C Nuclear Magnetic Resonance Spectroscopy. J Neurosci 23:5928-5935. doi:10.1523/JNEUROSCI.23-13-05928.2003

Edmond J, Robbins RA, Bergstrom JD, Cole RA, de Vellis J. 1987. Capacity for substrate utilization in oxidative metabolism by neurons, astrocytes, and oligodendrocytes from developing brain in primary culture. J Neurosci Res 18:551-561. doi:10.1002/jnr.490180407

Erwig MS, Patzig J, Steyer AM, Dibaj P, Heilmann M, Heilmann I, Jung RB, Kusch K, Möbius W, Jahn O, Nave K-A, Werner HB. 2019. Anillin facilitates septin assembly to prevent pathological outfoldings of central nervous system myelin. Elife 8:1-18. doi:10.7554/eLife.43888

Ferreirinha F, Quattrini A, Pirozzi M, Valsecchi V, Dina G, Broccoli V, Auricchio A, Piemonte F, Tozzi G, Gaeta L, Casari G, Ballabio A, Rugarli EI. 2004. Axonal degeneration in paraplegindeficient mice is associated with abnormal mitochondria and impairment of axonal transport. J Clin Invest 113:231-242. doi:10.1172/JCI200420138

Fünfschilling U, Supplie LM, Mahad D, Boretius S, Saab AS, Edgar J, Brinkmann BG, Kassmann CM, Tzvetanova ID, Möbius W, Diaz F, Meijer D, Suter U, Hamprecht B, Sereda MW, Moraes CT, Frahm J, Goebbels S, Nave K-A. 2012. Glycolytic oligodendrocytes maintain myelin and long-term axonal integrity. Nature 485:517-521. doi:10.1038/nature11007

Ghandour MS, Langley OK, Vincendon G, Gombos G, Filippi D, Limozin N, Dalmasso C, Laurent G. 1980. Immunochemical and immunohistochemical study of carbonic anhydrase II in adult rat cerebellum: A marker for oligodendrocytes. Neuroscience 5:559-571. doi:10.1016/03064522(80)90053-6

Glick D, Barth S, Macleod KF. 2010. Autophagy: cellular and molecular mechanisms. J Pathol 221:3-12. doi:10.1002/path.2697

Haces ML, Hernández-Fonseca K, Medina-Campos ON, Montiel T, Pedraza-Chaverri J, Massieu L. 2008. Antioxidant capacity contributes to protection of ketone bodies against oxidative damage induced during hypoglycemic conditions. Exp Neuro/ 211:85-96. doi:10.1016/j.expneurol.2007.12.029 
Harris JJ, Attwell D. 2012. The Energetics of CNS White Matter. J Neurosci 32:356-371. doi:10.1523/JNEUROSCI.3430-11.2012

Hartline DK, Colman DR. 2007. Rapid Conduction and the Evolution of Giant Axons and Myelinated Fibers. Curr Bio/ 17:R29-R35. doi:10.1016/j.cub.2006.11.042

He M, Rutledge SL, Kelly DR, Palmer CA, Murdoch G, Majumder N, Nicholls RD, Pei Z, Watkins PA, Vockley J. 2007. A New Genetic Disorder in Mitochondrial Fatty Acid $\beta$-Oxidation: ACAD9 Deficiency. Am J Hum Genet 81:87-103. doi:10.1086/519219

Hirrlinger J, Nave K-A. 2014a. Adapting brain metabolism to myelination and long-range signal transduction. Glia 62:1749-1761. doi:10.1002/glia.22737

Hirrlinger J, Nave K. 2014b. Adapting Brain Metabolism to Myelination and Long-Range Signal Transduction. doi:10.1002/glia.22737

Houten SM, Violante S, Ventura F V., Wanders RJA. 2016. The Biochemistry and Physiology of Mitochondrial Fatty Acid $\beta$-Oxidation and Its Genetic Disorders. Annu Rev Physio/ 78:23-44. doi:10.1146/annurev-physiol-021115-105045

Hovik R, Brodal B, Bartlett K, Osmundsen H. 1991. Metabolism of acetyl-CoA by isolated peroxisomal fractions: formation of acetate and acetoacetyl-CoA. J Lipid Res 32:993-9. Ioannou MS, Jackson J, Sheu S-H, Chang C-L, Weigel A V., Liu H, Pasolli HA, Xu CS, Pang S, Matthies D, Hess HF, Lippincott-Schwartz J, Liu Z. 2019. Neuron-Astrocyte Metabolic Coupling Protects against Activity-Induced Fatty Acid Toxicity. Cel/ 177:1522-1535.e14. doi:10.1016/j.cell.2019.04.001

Januszewicz E, Pająk B, Gajkowska B, Samluk Ł, Djavadian RL, Hinton BT, Nałęcz KA. 2009. Organic cation/carnitine transporter OCTN3 is present in astrocytes and is up-regulated by peroxisome proliferators-activator receptor agonist. Int J Biochem Cell Bio/41:2599-2609. doi:10.1016/j.biocel.2009.08.020

Jeremy M Berg, John L Tymoczko and LS. 2002. Biochemistry, 5th ed. New York: W.H. Freeman. Jung S, Aliberti J, Graemmel P, Sunshine MJ, Kreutzberg GW, Sher A, Littman DR. 2000. Analysis of Fractalkine Receptor CX3CR1 Function by Targeted Deletion and Green Fluorescent Protein Reporter Gene Insertion. Mol Cel/ Bio/ 20:4106-4114. doi:10.1128/MCB.20.11.41064114.2000

Karram K, Goebbels S, Schwab M, Jennissen K, Seifert G, Steinhäuser C, Nave K-A, Trotter J. 2008. NG2-expressing cells in the nervous system revealed by the NG2-EYFP-knockin mouse. genesis 46:743-757. doi:10.1002/dvg.20440

Kassmann CM. 2014. Myelin peroxisomes - Essential organelles for the maintenance of white matter in the nervous system. Biochimie 98:111-118. doi:10.1016/j.biochi.2013.09.020 
Kassmann CM, Lappe-Siefke C, Baes M, Brügger B, Mildner A, Werner HB, Natt O, Michaelis T, Prinz M, Frahm J, Nave K-A. 2007. Axonal loss and neuroinflammation caused by peroxisome-deficient oligodendrocytes. Nat Genet 39:969-976. doi:10.1038/ng2070

Kassmann CM, Quintes S, Rietdorf J, Möbius W, Sereda MW, Nientiedt T, Saher G, Baes M, Nave K-A. 2011. A role for myelin-associated peroxisomes in maintaining paranodal loops and axonal integrity. FEBS Lett 585:2205-2211. doi:10.1016/j.febslet.2011.05.032

Kihara A. 2012. Very long-chain fatty acids: Elongation, physiology and related disorders. J Biochem 152:387-395. doi:10.1093/jb/mvs105

Klosinski LP, Yao J, Yin F, Fonteh AN, Harrington MG, Christensen TA, Trushina E, Brinton RD. 2015. White Matter Lipids as a Ketogenic Fuel Supply in Aging Female Brain: Implications for Alzheimer's Disease. EBioMedicine 2:1888-1904. doi:10.1016/j.ebiom.2015.11.002

Kukley M, Capetillo-Zarate E, Dietrich D. 2007. Vesicular glutamate release from axons in white matter. Nat Neurosci 10:311-320. doi:10.1038/nn1850

Lamhonwah A-M, Ackerley CA, Tilups A, Edwards VD, Wanders RJ, Tein I. 2005. OCTN3 is a mammalian peroxisomal membrane carnitine transporter. Biochem Biophys Res Commun 338:1966-1972. doi:10.1016/j.bbrc.2005.10.170

Lappe-Siefke C, Goebbels S, Gravel M, Nicksch E, Lee J, Braun PE, Griffiths IR, Nave K-A. 2003. Disruption of Cnp1 uncouples oligodendroglial functions in axonal support and myelination. Nat Genet 33:366-374. doi:10.1038/ng1095

Lazarow PB. 1978. Rat liver peroxisomes catalyze the beta oxidation of fatty acids. J Bio/ Chem 253:1522-8.

Lebel C, Gee M, Camicioli R, Wieler M, Martin W, Beaulieu C. 2012. Diffusion tensor imaging of white matter tract evolution over the lifespan. Neuroimage 60:340-352. doi:10.1016/j.neuroimage.2011.11.094

Lee J, Wolfgang MJ. 2012. Metabolomic profiling reveals a role for CPT1c in neuronal oxidative metabolism. BMC Biochem 13:23. doi:10.1186/1471-2091-13-23

Lee Y, Morrison BM, Li Y, Lengacher S, Farah MH, Hoffman PN, Liu Y, Tsingalia A, Jin L, Zhang PW, Pellerin L, Magistretti PJ, Rothstein JD. 2012. Oligodendroglia metabolically support axons and contribute to neurodegeneration. Nature 487:443-448. doi:10.1038/nature11314

Leone DP, Genoud S téphan., Atanasoski S, Grausenburger R, Berger P, Metzger D, Macklin WB, Chambon P, Suter U. 2003. Tamoxifen-inducible glia-specific Cre mice for somatic mutagenesis in oligodendrocytes and Schwann cells. Mol Cell Neurosci 22:430-440. doi:10.1016/S1044-7431(03)00029-0

Lodish H, Berk A, Zipursky SL et al. 2000. Molecular Cell Biology, 4th ed. New York: W. H. 
Freeman.

Longo N, Frigeni M, Pasquali M. 2016. Carnitine transport and fatty acid oxidation. Biochim Biophys Acta - Mol Cell Res 1863:2422-2435. doi:10.1016/j.bbamcr.2016.01.023

LOSKOVICH M V., GRIVENNIKOVA VG, CECCHINI G, VINOGRADOV AD. 2005. Inhibitory effect of palmitate on the mitochondrial NADH:ubiquinone oxidoreductase (complex I) as related to the active-de-active enzyme transition. Biochem J 387:677-683. doi:10.1042/BJ20041703

LOWRY OH, ROSEBROUGH NJ, FARR AL, RANDALL RJ. 1951. Protein measurement with the Folin phenol reagent. J Biol Chem 193:265-75.

Makowski L, Hotamisligil GS. 2004. Fatty Acid Binding Proteins-The Evolutionary Crossroads of Inflammatory and Metabolic Responses. J Nutr 134:2464S-2468S. doi:10.1093/jn/134.9.2464s

Mitchell RW, On NH, Del Bigio MR, Miller DW, Hatch GM. 2011. Fatty acid transport protein expression in human brain and potential role in fatty acid transport across human brain microvessel endothelial cells. J Neurochem 117:735-746. doi:10.1111/j.14714159.2011.07245.x

Moody DM, Bell MA, Challa VR. 1990. Features of the cerebral vascular pattern that predict vulnerability to perfusion or oxygenation deficiency: an anatomic study. AJNR Am J Neuroradio/11:431-9.

Mosconi L. 2005. Brain glucose metabolism in the early and specific diagnosis of Alzheimer?s disease. Eur J Nucl Med Mol Imaging 32:486-510. doi:10.1007/s00259-005-1762-7

Nave KA. 2010. Myelination and support of axonal integrity by glia. Nature 468:244-252. doi:10.1038/nature09614

Newman JC, Verdin E. 2017. $\beta$-Hydroxybutyrate: A Signaling Metabolite. Annu Rev Nutr 37:5176. doi:10.1146/annurev-nutr-071816-064916

Nickel K, Tebartz van Elst L, Holovics L, Feige B, Glauche V, Fortenbacher T, Endres D, Zeeck A, Tüscher O, Joos A, Maier S. 2019. White Matter Abnormalities in the Corpus Callosum in Acute and Recovered Anorexia Nervosa Patients-A Diffusion Tensor Imaging Study. Front Psychiatry 10:1-10. doi:10.3389/fpsyt.2019.00490

Nishimoto Y, Tamori Y. 2017. CIDE Family-Mediated Unique Lipid Droplet Morphology in White Adipose Tissue and Brown Adipose Tissue Determines the Adipocyte Energy Metabolism. J Atheroscler Thromb 24:989-998. doi:10.5551/jat.RV17011

NOVIKOFF AB, GOLDFISCHER S. 1969. VISUALIZATION OF PEROXISOMES (MICROBODIES) AND MITOCHONDRIA WITH DIAMINOBENZIDINE. J Histochem Cytochem 17:675-680. doi:10.1177/17.10.675 
Olowe Y, Schulz H. 1982. 4-Bromocrotonic acid, an effective inhibitor of fatty acid oxidation and ketone body degradation in rat heart mitochondria. On the rate-determining step of betaoxidation and ketone body degradation in heart. J Biol Chem 257:5408-13.

Owen OE, Morgan AP, Kemp HG, Sullivan JM, Herrera MG, Cahill GF. 1967. Brain Metabolism during Fasting*. J Clin Invest 46:1589-1595. doi:10.1172/JCI105650

Ozgen H, Baron W, Hoekstra D, Kahya N. 2016. Oligodendroglial membrane dynamics in relation to myelin biogenesis. Cell Mol Life Sci 73:3291-3310. doi:10.1007/s00018-016-2228-8

Panov A, Orynbayeva Z, Vavilin V, Lyakhovich V. 2014. Fatty Acids in Energy Metabolism of the Central Nervous System. Biomed Res Int 2014:1-22. doi:10.1155/2014/472459

Pellerin L, Magistretti PJ. 1994. Glutamate uptake into astrocytes stimulates aerobic glycolysis: a mechanism coupling neuronal activity to glucose utilization. Proc Nat/ Acad Sci 91:1062510629. doi:10.1073/pnas.91.22.10625

PLOMP PJAM, WOLVETANG EJ, GROEN AK, MEIJER AJ, GORDON PB, SEGLEN PO. 1987. Energy dependence of autophagic protein degradation in isolated rat hepatocytes. Eur J Biochem 164:197-203. doi:10.1111/j.1432-1033.1987.tb11011.x

Pohl J, Ring A, Ehehalt R, Schulze-Bergkamen H, Schad A, Verkade P, Stremmel W. 2004. LongChain Fatty Acid Uptake into Adipocytes Depends on Lipid Raft Function. Biochemistry 43:4179-4187. doi:10.1021/bi035743m

Pohl J, Ring A, Korkmaz Ü, Ehehalt R, Stremmel W. 2005. FAT/CD36-mediated Long-Chain Fatty Acid Uptake in Adipocytes Requires Plasma Membrane Rafts. Mol Biol Cel/ 16:24-31. doi:10.1091/mbc.e04-07-0616

POOLE RC, SANSOM CE, HALESTRAP AP. 1996. Studies of the membrane topology of the rat erythrocyte $\mathrm{H}+$ /lactate cotransporter (MCT1). Biochem / 320:817-824. doi:10.1042/bj3200817

Richert S, Kleinecke S, Günther J, Schaumburg F, Edgar J, Nienhaus GU, Nave K-A, Kassmann CM. 2014. In vivo labeling of peroxisomes by photoconvertible mEos2 in myelinating glia of mice. Biochimie 98:127-134. doi:10.1016/j.biochi.2013.10.022

Ring A, Le Lay S, Pohl J, Verkade P, Stremmel W. 2006. Caveolin-1 is required for fatty acid translocase (FAT/CD36) localization and function at the plasma membrane of mouse embryonic fibroblasts. Biochim Biophys Acta - Mol Cell Biol Lipids 1761:416-423. doi:10.1016/j.bbalip.2006.03.016

Roa-Mansergas X, Fadó R, Atari M, Mir JF, Muley H, Serra D, Casals N. 2018. CPT1C promotes human mesenchymal stem cells survival under glucose deprivation through the modulation of autophagy. Sci Rep 8:6997. doi:10.1038/s41598-018-25485-7 
Rouiller C, Bernhard W. 1956. "MICROBODIES" AND THE PROBLEM OF MITOCHONDRIAL REGENERATION IN LIVER CELLS. J Biophys Biochem Cyto/ 2:355-360. doi:10.1083/jcb.2.4.355

Saab AS, Tzvetavona ID, Trevisiol A, Baltan S, Dibaj P, Kusch K, Möbius W, Goetze B, Jahn HM, Huang W, Steffens $H$, Schomburg ED, Pérez-Samartín A, Pérez-Cerdá F, Bakhtiari D, Matute C, Löwel S, Griesinger C, Hirrlinger J, Kirchhoff F, Nave K-A. 2016. Oligodendroglial NMDA Receptors Regulate Glucose Import and Axonal Energy Metabolism. Neuron 91:119-132. doi:10.1016/j.neuron.2016.05.016

Sardiello M, Palmieri M, di Ronza A, Medina DL, Valenza M, Gennarino VA, Di Malta C, Donaudy F, Embrione V, Polishchuk RS, Banfi S, Parenti G, Cattaneo E, Ballabio A. 2009. A Gene Network Regulating Lysosomal Biogenesis and Function. Science (80- ) 325:473-477. doi:10.1126/science.1174447

Schiff M, Haberberger B, Xia C, Mohsen A-W, Goetzman ES, Wang Y, Uppala R, Zhang Y, Karunanidhi A, Prabhu D, Alharbi H, Prochownik E V., Haack T, Häberle J, Munnich A, Rötig A, Taylor RW, Nicholls RD, Kim J-J, Prokisch H, Vockley J. 2015. Complex I assembly function and fatty acid oxidation enzyme activity of ACAD9 both contribute to disease severity in ACAD9 deficiency. Hum Mol Genet 24:3238-3247. doi:10.1093/hmg/ddv074

Schlaepfer IR, Glodé LM, Hitz CA, Pac CT, Boyle KE, Maroni P, Deep G, Agarwal R, Lucia SM, Cramer SD, Serkova NJ, Eckel RH. 2015. Inhibition of Lipid Oxidation Increases Glucose Metabolism and Enhances 2-Deoxy-2-[18F]Fluoro-d-Glucose Uptake in Prostate Cancer Mouse Xenografts. Mol Imaging Bio/ 17:529-538. doi:10.1007/s11307-014-0814-4

Schönfeld P, Reiser G. 2017. Inhibition of $\beta$-oxidation is not a valid therapeutic tool for reducing oxidative stress in conditions of neurodegeneration. J Cereb Blood Flow Metab 37:848-854. doi:10.1177/0271678X16642448

Schönfeld P, Reiser G. 2013. Why does Brain Metabolism not Favor Burning of Fatty Acids to Provide Energy? - Reflections on Disadvantages of the Use of Free Fatty Acids as Fuel for Brain. J Cereb Blood Flow Metab 33:1493-1499. doi:10.1038/jcbfm.2013.128

Schönfeld P, Wojtczak L. 2016. Short- and medium-chain fatty acids in energy metabolism: the cellular perspective. J Lipid Res 57:943-954. doi:10.1194/jlr.R067629

Schönfeld P, Wojtczak L. 2007. Fatty acids decrease mitochondrial generation of reactive oxygen species at the reverse electron transport but increase it at the forward transport. Biochim Biophys Acta - Bioenerg 1767:1032-1040. doi:10.1016/j.bbabio.2007.04.005

Schrader M, Fahimi HD. 2008a. The peroxisome: still a mysterious organelle. Histochem Cell Biol 129:421-440. doi:10.1007/s00418-008-0396-9 
Schrader M, Fahimi HD. 2008b. The peroxisome: still a mysterious organelle. Histochem Cell Biol 129:421-440. doi:10.1007/s00418-008-0396-9

Schulz JG, Laranjeira A, Van Huffel L, Gärtner A, Vilain S, Bastianen J, Van Veldhoven PP, Dotti CG. 2015. Glial $\beta$-Oxidation regulates Drosophila Energy Metabolism. Sci Rep 5:7805. doi:10.1038/srep07805

Settembre C, Ballabio A. 2014. Lysosome: regulator of lipid degradation pathways. Trends Cell Bio/24:743-750. doi:10.1016/j.tcb.2014.06.006

Settembre C, Di Malta C, Polito VA, Arencibia MG, Vetrini F, Erdin S, Erdin SU, Huynh T, Medina D, Colella P, Sardiello M, Rubinsztein DC, Ballabio A. 2011. TFEB Links Autophagy to Lysosomal Biogenesis. Science (80- ) 332:1429-1433. doi:10.1126/science.1204592

Settembre C, Zoncu R, Medina DL, Vetrini F, Erdin Serkan, Erdin Serpiluckac, Huynh T, Ferron M, Karsenty G, Vellard MC, Facchinetti V, Sabatini DM, Ballabio A. 2012. A lysosome-to-nucleus signalling mechanism senses and regulates the lysosome via mTOR and TFEB. EMBO J 31:1095-1108. doi:10.1038/emboj.2012.32

Simons M, Nave KA. 2016. Oligodendrocytes: Myelination and axonal support. Cold Spring Harb Perspect Bio/ 8:1-15. doi:10.1101/cshperspect.a020479

Sokoloff L, Clarke DD. 1989. Circulation and energy metabolism of the brain. Basic Neurochem 4th:565-590.

Sørensen BK, Højrup P, Østergård E, Jørgensen CS, Enghild J, Ryder LR, Houen G. 2002. Silver Staining of Proteins on Electroblotting Membranes and Intensification of Silver Staining of Proteins Separated by Polyacrylamide Gel Electrophoresis. Anal Biochem 304:33-41. doi:10.1006/abio.2001.5604

Stremmel W, Strohmeyer G, Berk PD. 1986. Hepatocellular uptake of oleate is energy dependent, sodium linked, and inhibited by an antibody to a hepatocyte plasma membrane fatty acid binding protein. Proc Natl Acad Sci 83:3584-3588. doi:10.1073/pnas.83.11.3584

Stys PK, Ransom BR, Waxman SG. 1991. Compound action potential of nerve recorded by suction electrode: a theoretical and experimental analysis. Brain Res 546:18-32. doi:10.1016/0006-8993(91)91154-S

Sun LO, Mulinyawe SB, Collins HY, Ibrahim A, Li Q, Simon DJ, Tessier-Lavigne M, Barres BA. 2018. Spatiotemporal Control of CNS Myelination by Oligodendrocyte Programmed Cell Death through the TFEB-PUMA Axis. Cel/175:1811-1826.e21. doi:10.1016/j.cell.2018.10.044

Swayze VW, Andersen AE, Andreasen NC, Arndt S, Sato Y, Ziebell S. 2003. Brain tissue volume segmentation in patients with anorexia nervosa before and after weight normalization. Int $J$ Eat Disord 33:33-44. doi:10.1002/eat.10111 
Swigoňová Z, Mohsen A-W, Vockley J. 2009. Acyl-CoA Dehydrogenases: Dynamic History of Protein Family Evolution. J Mol Evo/69:176-193. doi:10.1007/s00239-009-9263-0

Takeuchi Y, Morii H, Tamura M, Hayaishi O, Watanabe Y. 1991. A possible mechanism of mitochondrial dysfunction during cerebral ischemia: Inhibition of mitochondrial respiration activity by arachidonic acid. Arch Biochem Biophys 289:33-38. doi:10.1016/00039861(91)90438-O

Theodoulou FL, Carrier DJ, Schaedler TA, Baldwin SA, Baker A. 2016. How to move an amphipathic molecule across a lipid bilayer: different mechanisms for different $A B C$ transporters? Biochem Soc Trans 44:774-782. doi:10.1042/BST20160040

Titorenko V. 2001. Dynamics of peroxisome assembly and function. Trends Cell Bio/ 11:22-29. doi:10.1016/S0962-8924(00)01865-1

Tracey TJ, Steyn FJ, Wolvetang EJ, Ngo ST. 2018. Neuronal Lipid Metabolism: Multiple Pathways Driving Functional Outcomes in Health and Disease. Front Mol Neurosci 11:1-10. doi:10.3389/fnmol.2018.00010

Trevisiol A, Saab AS, Winkler U, Marx G, Imamura H, Möbius W, Kusch K, Nave K-A, Hirrlinger J. 2017. Monitoring ATP dynamics in electrically active white matter tracts. Elife 6:1-17. doi:10.7554/eLife.24241

Van den Branden C, Roels F. 1985. Thioridazine: a selective inhibitor of peroxisomal $\beta$-oxidation in vivo. FEBS Lett 187:331-333. doi:10.1016/0014-5793(85)81270-9

Vergnes L, Chin R, Young SG, Reue K. 2011. Heart-type Fatty Acid-binding Protein Is Essential for Efficient Brown Adipose Tissue Fatty Acid Oxidation and Cold Tolerance. J Biol Chem 286:380-390. doi:10.1074/jbc.M110.184754

Verheijden S, Bottelbergs A, Krysko O, Krysko D V., Beckers L, De Munter S, Van Veldhoven PP, Wyns S, Kulik W, Nave K-A, Ramer MS, Carmeliet P, Kassmann CM, Baes M. 2013. Peroxisomal multifunctional protein-2 deficiency causes neuroinflammation and degeneration of Purkinje cells independent of very long chain fatty acid accumulation. Neurobiol Dis 58:258-269. doi:10.1016/j.nbd.2013.06.006

Vignais PM, Gallagher CH, Zabin I. 1958. ACTIVATION AND OXIDATION OF LONG CHAIN FATTY ACIDS BY RAT BRAIN. J Neurochem 2:283-287. doi:10.1111/j.1471-4159.1958.tb12375.x

Violante S, IJst L, te Brinke H, Koster J, Tavares de Almeida I, Wanders RJA, Ventura F V., Houten SM. 2013. Peroxisomes contribute to the acylcarnitine production when the carnitine shuttle is deficient. Biochim Biophys Acta - Mol Cell Biol Lipids 1831:1467-1474. doi:10.1016/j.bbalip.2013.06.007

Wanders RJA, Ruiter JPN, IJIst L, Waterham HR, Houten SM. 2010. The enzymology of 
mitochondrial fatty acid beta-oxidation and its application to follow-up analysis of positive neonatal screening results. J Inherit Metab Dis 33:479-494. doi:10.1007/s10545-010-9104-8 Wanders RJA, Waterham HR. 2006. Biochemistry of Mammalian Peroxisomes Revisited. Annu Rev Biochem 75:295-332. doi:10.1146/annurev.biochem.74.082803.133329

Wanders RJA, Waterham HR, Ferdinandusse S. 2016. Metabolic Interplay between Peroxisomes and Other Subcellular Organelles Including Mitochondria and the Endoplasmic Reticulum. Front Cell Dev Bio/ 3:1-15. doi:10.3389/fcell.2015.00083

Waxman SG. 1997. Axon-glia interactions: Building a smart nerve fiber. Curr Bio/ 7:R406-R410. doi:10.1016/S0960-9822(06)00203-X

Weil M-T, Heibeck S, Töpperwien M, tom Dieck S, Ruhwedel T, Salditt T, Rodicio MC, Morgan JR, Nave K-A, Möbius W, Werner HB. 2018. Axonal Ensheathment in the Nervous System of Lamprey: Implications for the Evolution of Myelinating Glia. J Neurosci 38:6586-6596. doi:10.1523/JNEUROSCI.1034-18.2018

Welte MA, Gould AP. 2017. Lipid droplet functions beyond energy storage. Biochim Biophys Acta - Mol Cell Biol Lipids 1862:1260-1272. doi:10.1016/j.bbalip.2017.07.006

Wender R, Brown AM, Fern R, Swanson RA, Farrell K, Ransom BR. 2000. Astrocytic Glycogen Influences Axon Function and Survival during Glucose Deprivation in Central White Matter. J Neurosci 20:6804-6810. doi:10.1523/JNEUROSCI.20-18-06804.2000

Westin MAK, Hunt MC, Alexson SEH. 2008. Short- and medium-chain carnitine acyltransferases and acyl-CoA thioesterases in mouse provide complementary systems for transport of $\beta$ oxidation products out of peroxisomes. Cell Mol Life Sci 65:982-990. doi:10.1007/s00018008-7576-6

Wolfgang M. 2016. Carnitine Palmitoyltransfersase-1c and 5-oxoprolinase interact in the mouse brain.\&amp;nbsp; \&amp;nbsp; \&amp;nbsp; \&amp;nbsp; Matters 1-4. doi:10.19185/matters.201609000009

Yang SY, He XY, Schulz H. 1987. Fatty acid oxidation in rat brain is limited by the low activity of 3-ketoacyl-coenzyme A thiolase. J Biol Chem 262:13027-32.

Yang X, Sun L, Zhao A, Hu X, Qing Y, Jiang J, Yang C, Xu T, Wang P, Liu J, Zhang J, He L, Jia W, Wan C. 2017. Serum fatty acid patterns in patients with schizophrenia: a targeted metabonomics study. Trans/ Psychiatry 7:e1176-e1176. doi:10.1038/tp.2017.152

Yao J, Hamilton RT, Cadenas E, Brinton RD. 2010. Decline in mitochondrial bioenergetics and shift to ketogenic profile in brain during reproductive senescence. Biochim Biophys Acta Gen Subj 1800:1121-1126. doi:10.1016/j.bbagen.2010.06.002

Yao J, Irwin RW, Zhao L, Nilsen J, Hamilton RT, Brinton RD. 2009. Mitochondrial bioenergetic 
deficit precedes Alzheimer's pathology in female mouse model of Alzheimer's disease. Proc Natl Acad Sci 106:14670-14675. doi:10.1073/pnas.0903563106

Yao J, Rettberg JR, Klosinski LP, Cadenas E, Brinton RD. 2011. Shift in brain metabolism in late onset Alzheimer's disease: Implications for biomarkers and therapeutic interventions. $\mathrm{Mo} /$ Aspects Med 32:247-257. doi:10.1016/j.mam.2011.10.005

Young CD, Lewis AS, Rudolph MC, Ruehle MD, Jackman MR, Yun UJ, Ilkun O, Pereira R, Abel ED, Anderson SM. 2011. Modulation of Glucose Transporter 1 (GLUT1) Expression Levels Alters Mouse Mammary Tumor Cell Growth In Vitro and In Vivo. PLoS One 6:e23205. doi:10.1371/journal.pone.0023205

Zechner R, Zimmermann R, Eichmann TO, Kohlwein SD, Haemmerle G, Lass A, Madeo F. 2012. FAT SIGNALS - Lipases and Lipolysis in Lipid Metabolism and Signaling. Cell Metab 15:279291. doi:10.1016/j.cmet.2011.12.018

Zhang J, Zhang W, Zou D, Chen G, Wan T, Zhang M, Cao X. 2002. Cloning and functional characterization of ACAD-9, a novel member of human acyl-CoA dehydrogenase family. Biochem Biophys Res Commun 297:1033-1042. doi:10.1016/S0006-291X(02)02336-7

Zhang L, Dean D, Liu JZ, Sahgal V, Wang X, Yue GH. 2007. Quantifying degeneration of white matter in normal aging using fractal dimension. Neurobiol Aging 28:1543-1555. doi:10.1016/j.neurobiolaging.2006.06.020

Ziskin JL, Nishiyama A, Rubio M, Fukaya M, Bergles DE. 2007. Vesicular release of glutamate from unmyelinated axons in white matter. Nat Neurosci 10:321-330. doi:10.1038/nn1854 


\section{Kurzfassung (Abstract in German)}

Die neuronalen Funktionen hängen entscheidend von einer kontinuierlichen Glukosezufuhr ab, da eine anhaltende Hypoglykämie zu schweren Hirnschädigungen führen kann. Es ist überraschend, dass das Gehirn außer des astroglialen Glykogens keine sichtbaren Energiereserven besitzt. Myelin ist eine lipidreiche Membran, die Axone zur schnellen Reizleitung umhüllt. Myelinisierende Oligodendrozyten unterstützen auch den Stoffwechsel der Axone. Wir stellten daher die Hypothese auf, dass unter Hungerbedingungen, das Myelin selbst den Energiehaushalt der weißen Substanz unterstützen könnte. Am Modell des Sehnervs der Maus fanden wir heraus, dass Oligodendrozyten, nicht aber Astrozyten, den Glukoseentzug ex vivo bis zu 24 Stunden überleben. Darüber hinaus wurde beobachtet, dass die Myelindicke unter Hungerbedingungen abnimmt, unter anderem konnte gezeigt werden, dass die Hemmung der Beta-Oxidation einen massiven Zelltod im Sehnerv induziert. Diese Beobachtungen deuten darauf hin, dass aus Myelin freigesetzte Fettsäuren an der Unterstützung des Zellüberlebens unter Hungerbedingungen beteiligt sind. Maßgeblich ist, dass wir mittels Elektrophysiologie in Kombination mit Live-Bildgebung zeigten, dass der Fettsäurestoffwechsel die axonale ATP-Produktion und die Nervenleitung durch Störung der Beta-Oxidation unterstützt. Diese axonale Unterstützung hängt von der Fettsäure-BetaOxidation ab, an der Peroxisomen im Myelinkompartiment beteiligt sind. Um die reduzierte Verfügbarkeit von Glukose in lebenden Mäusen zu untersuchen, haben wir GLUT1 (Slc2a1) aus reifen Oligodendrozyten entfernt, was zu einem langsam fortschreitenden Myelinverlust führte. Wir schlagen daher ein überarbeitetes Modell für die Doppelfunktion der Oligodendrozyten vor, bei dem Myelin einen Energiepuffer bildet, der die axonale Degeneration verhindern kann, wenn die Glukoseverfügbarkeit gestört ist. Diese Ergebnisse könnten für Myelin-Anomalien relevant sein, die bei einer Reihe von neurodegenerativen Erkrankungen auftreten. 